\title{
A CLASS OF GLOBALLY SOLVABLE MARKOVIAN QUADRATIC BSDE SYSTEMS AND APPLICATIONS
}

\author{
By HAO XING AND GORDAN ŽITKOVIĆ ${ }^{1}$
}

\section{London School of Economics and University of Texas at Austin}

We establish existence and uniqueness for a wide class of Markovian systems of backward stochastic differential equations (BSDE) with quadratic nonlinearities. This class is characterized by an abstract structural assumption on the generator, an a priori local-boundedness property, and a locallyHölder-continuous terminal condition. We present easily verifiable sufficient conditions for these assumptions and treat several applications, including stochastic equilibria in incomplete financial markets, stochastic differential games and martingales on Riemannian manifolds.

\section{CONTENTS}

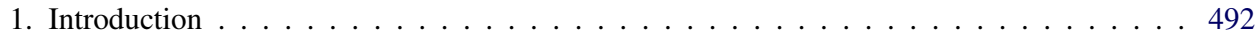

1.1. Backward stochastic differential equations . . . . . . . . . . . . . . . . 492

1.2. Our contributions-The main result . . . . . . . . . . . . . . . . . . . . 493

1.3. Our results-Sufficient conditions and examples . . . . . . . . . . . . . . . 495

1.4. Structure of the paper . . . . . . . . . . . . . . . . . . . . 495

1.5. Notation and conventions . . . . . . . . . . . . . . . . . . . 496

2. Main results . . . . . . . . . . . . . . . . . . . . . . . . . . . 497

2.1. The setup, standing assumptions and key concepts . . . . . . . . . . . . . . . 497

2.1.1. The driving diffusion . . . . . . . . . . . . . . . . . . . . . . . 497

2.1.2. Markovian and Hölderian solutions _. . . . . . . . . . . . . . . . . . 497

2.1.3. Lyapunov functions . . . . . . . . . . . . . . . . . . . . . . . 498

2.2. A uniform estimate . . . . . . . . . . . . . . . . . . . . . . . . . . . . 499

2.3. Existence and uniqueness . . . . . . . . . . . . . . . . . . . 501

2.4. A sufficient condition for existence and uniqueness . . . . . . . . . . . 502

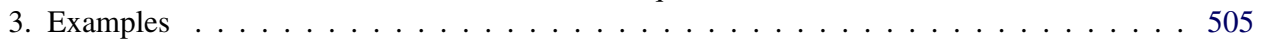

3.1. Incomplete stochastic equilibria . . . . . . . . . . . . . . . . 505

3.2. Martingales on manifolds . . . . . . . . . . . . . . . . . . . . . . 506

3.3. A stochastic game with cooperation or hindrance . . . . . . . . . . . . . . . . . 509

3.4. Risk-sensitive nonzero-sum stochastic games . . . . . . . . . . . . . . . . 510

3.5. A scalar example with unbounded coefficients . . . . . . . . . . . . . . . . 511

Received March 2016; revised March 2017.

${ }^{1}$ Supported by NSF Grants DMS-11-07465 (2012-2017) and DMS-15-16165 (2015-2018). Any opinions, findings and conclusions or recommendations expressed in this material are those of the author(s) and do not necessarily reflect the views of the National Science Foundation (NSF).

MSC2010 subject classifications. Primary 60G44, 60G99, 60H30; secondary 58J65, 91A15, 91B51.

Key words and phrases. BSDE, backward stochastic differential equations, systems of BSDE, quadratic nonlinearities, stochastic equilibrium, martingales on manifolds, nonzero-sum stochastic games. 
4. Proof of Theorem $2.5 \ldots \ldots \ldots \ldots \ldots \ldots \ldots \ldots \ldots \ldots$

4.1. A "testing" lemma . . . . . . . . . . . . . . . . . . . . 513

4.2. First consequences of the regularity of transition densities . . . . . . . . . . . . 5 515

4.3. Uniform local estimates . . . . . . . . . . . . . . . . . . . . . . 518

4.4. A weighted Poincaré inequality and Struwe's lemma . . . . . . . . . . . . . . . 5 522

4.5. Hole-filling . . . . . . . . . . . . . . . . . . . . . . . . 528

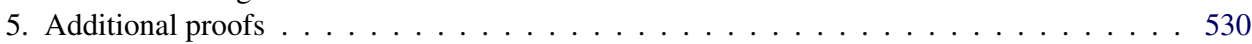

5.1. Proof of Theorem $2.8 \ldots \ldots \ldots \ldots \ldots \ldots$

5.2. Proof of Theorem $2.9 \ldots \ldots \ldots \ldots \ldots \ldots \ldots$

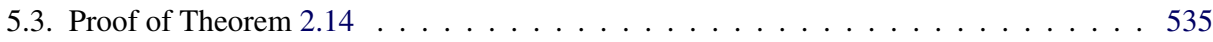

5.3.1. Lipschitz approximations . . . . . . . . . . . . . . . 535

5.3.2. Existence of Lyapunov pairs . . . . . . . . . . . . . . . . . 538

5.3.3. Conclusion of the Proof of Theorem $2.14 \ldots \ldots \ldots$. . . . . . . . . 541

5.4. Proofs for examples . . . . . . . . . . . . . . . . . . . . 543

5.4.1. Proof of Theorem $3.1 \ldots \ldots \ldots \ldots \ldots \ldots$

5.4.2. Proof of Proposition $3.4 \ldots \ldots \ldots \ldots \ldots$

5.4.3. Proof of Proposition $3.6 \ldots \ldots \ldots \ldots \ldots 4 \ldots \ldots \ldots$

5.4.4. Proof of Proposition $3.7 \ldots \ldots \ldots \ldots \ldots 4 \ldots \ldots \ldots$

Acknowledgment . . . . . . . . . . . . . . . . . . . . . 547

References . . . . . . . . . . . . . . . . . . . . . . . . 548

\section{Introduction.}

1.1. Backward stochastic differential equations. Having appeared first in their linear variant in [8], backward stochastic differential equations (BSDE) have been a subject of extensive study since the seminal paper [50]. Given a time horizon $T \in(0, \infty)$ and a filtered probability space $\left(\Omega, \mathcal{F},\left\{\mathcal{F}_{t}\right\}_{t \in[0, T]}, \mathbb{P}\right)$, which satisfies the usual conditions, these equations take the following form:

$$
\boldsymbol{Y}_{t}=\boldsymbol{G}+\int_{t}^{T} \boldsymbol{f}\left(s, \boldsymbol{Y}_{s}, \boldsymbol{Z}_{s}\right) d s-\int_{t}^{T} \boldsymbol{Z}_{s} d W_{s}
$$

where $W$ is a $d$-dimensional $\left\{\mathcal{F}_{t}\right\}_{t \in[0, T]}$-Brownian motion, $\boldsymbol{G} \in \mathcal{F}_{T}$ an $N$ dimensional random vector and $\boldsymbol{f}$ a (possibly random) function, called the generator. A solution to such an equation is a pair $(\boldsymbol{Y}, \boldsymbol{Z})$ consisting of an $N$-dimensional semimartingale $\boldsymbol{Y}$ and an $N \times d$-dimensional adapted process $\boldsymbol{Z}$ which together satisfy (1.1) pathwise, a.s.

The existence and uniqueness theory is well developed in the scalar $(N=1)$ setting. It originated with the Lipschitz-generator case treated in [50], continued in [46] for merely continuous generators with linear growth,and culminated with the treatment of quadratic nonlinearities in [42] and superquadratic nonlinearities in [24]. A host of extensions, simplifications and applications, too numerous to list here, appeared in the literature since.

On the other hand, systems $(N>1)$ of BSDE-the focus of the present paperpose a greater challenge. Their successful treatment is one of the most important (and long-standing) open problems in the entire theory, as mentioned already 
by Peng in [51]. While the case of a Lipschitz generator was treated already in [50], the general, nonlinear, quadratically-growing case is still open. One of the most well-known general-purpose results has been established in [56], where the generator has general quadratic growth, but the terminal condition is subject to a restrictive "smallness" assumption imposed on its $\mathbb{L}^{\infty}$-norm. As is the case in the theory of systems of parabolic PDEs with quadratic nonlinearities, a smallness assumption is often made and seems to be necessary for existence in full generality, in absence of any further, structural assumptions; cf. [53] and [15]. A simple nonexistence example given in [33] illustrates this point quite effectively.

Positive results without any smallness assumptions have been established in some special cases. Focusing only on the general existence results in the multidimensional case pertinent to our findings, let us mention just a few: [55] deals with linear-quadratic systems, [18] builds a structure around the ability to change the probability measure in the Markovian case and in [37], a slightly less general, "diagonally"-quadratic case is treated, but without the Markovian assumption.

Our present work was motivated not only by the unresolved status of the basic existence and uniqueness problems for quadratic systems of BSDE, but also by a number of applications such systems have. Indeed, in addition to their innate mathematical interest, BSDE appear in numerous applications, including stochastic representations for partial differential equations, optimal stochastic control and stochastic games (see, e.g., [16, 26, 29] and [43]). Moreover, as has been shown in [40], arguably the most important open problem in stochastic financial economics - namely, the so-called incomplete-market equilibrium problem-can be reduced to a quadratic system of BSDE (which we solve in the present paper). Quadratic systems of BSDE also appear in geometry, most prominently in the study of harmonic functions between manifolds and the construction of martingales on curved spaces, (see, e.g., $[9,21]$ and [10]).

1.2. Our contributions-The main result. We focus on a Markovian framework, where the randomness in the generator and the terminal condition is supplied by a (forward) $d$-dimensional nondegenerate diffusion $X$. Our terminal condition is of the form $\boldsymbol{G}=\boldsymbol{g}\left(X_{T}\right)$ and the generator $\boldsymbol{f}\left(t, X_{t}, \boldsymbol{Y}_{t}, \boldsymbol{Z}_{t}\right)$ grows at most quadratically in $\boldsymbol{Z}$. We formulate and work with a novel structural condition on $\boldsymbol{f}$, which requires the existence of what we term a Lyapunov function. Loosely speaking, a Lyapunov function $h$ has the property that $h(\boldsymbol{Y})$ is a "strict" submartingale, a priori, for any solution $\boldsymbol{Y}$ of (1.1) (actually, we consider a pair of functions, but we focus on only one of them in this Introduction). Under the quadratic-growth assumption, Lyapunov functions always exist in the 1-dimensional case and can be found in the class of exponential functions; this explains the success of the exponential transform in the 1-dimensional setting. The multidimensional case appears 
to be much more difficult, but as we show, widely applicable sufficient conditions can be given.

Our main result states that when a Lyapunov function exists and an additional a priori local-boundedness condition holds, the equation (1.1) admits a Markovian solution as soon as $\boldsymbol{g}$ belongs to an appropriately-defined local Hölder space, without any assumptions of the "smallness" type on the terminal condition, the driver or the time horizon. Moreover, under an additional mild assumption, this solution turns out to be unique in a wide class of stochastic processes.

In contrast to the bulk of the literature on multidimensional BSDE, we rely on deep analytic results for systems of PDEs and combine them with probabilistic techniques. More precisely, we use powerful ideas first introduced to study regularity theory for systems of parabolic PDEs, most notably the so-called partialregularity estimates and the hole-filling technique of [58], initially developed for elliptic systems and later extended to parabolic systems of PDEs in [53], which was later used in [7]. Partial-regularity and hole-filling techniques can be seen as a replacement for order-based arguments involving, for instance, the maximum principle (comparison principle) or the related notion of a viscosity solution; such methods, unfortunately, fail miserably in the multidimensional case (see [36]). However, we represent this analytic theory entirely in a probabilistic fashion. This allows us to implement the hole-filling technique only assuming the existence of a Lyapunov function. This strategy not only decouples the hole-filling technique from specific structural conditions on the nonlinearity such as the "smallness" condition in [53] and the structural condition in [7]. It also links naturally to the notion of geodesic convexity in the studies of martingales on manifolds (see Section 3.2). The probabilistic treatment also reduces some technical estimates from its the analytic counterpart. In particular, by replacing integration-by-parts techniques by Itô's formula, we bypass the estimates on derivatives of Gaussian transition densities present in [53] and [7].

A major difficulty in adopting the techniques from the theory of systems of PDE lies in the choice of the regularity class of the Markov representatives, that is, functions $\boldsymbol{v}$ such that $\boldsymbol{Y}_{t}=\boldsymbol{v}\left(t, X_{t}\right)$ is a solution. On one hand, the classical notion of a weak solution - typically a starting point for any regularity analysis in the PDE world - is too weak for us; indeed, the very definition of a solution to a BSDE requires $\boldsymbol{Y}$ to be a semimartingale (see, however, [3, 4, 45] and [48] for developments in Lipschitz systems). On the opposite end of the spectrum, a classical $C^{1,2}$ solution would, indeed, guarantee the semimartingale property of $\boldsymbol{v}\left(t, X_{t}\right)$, but one can hardly expect that kind of regularity from a solution to a nonlinear system. In the one-dimensional case, the situation is fully understood-Markov representatives of solutions to Markovian BSDE in dimension 1 are viscosity solutions to the associated quasilinear PDEs (see [13, 42, 49] or [24]). The multidimensional case, again, presents major difficulties: unless the system is very weakly coupled (only in its zero-th order terms), there is no natural notion of a viscosity solution and there is no corresponding characterization of the class of semimartingale 
functions (see, however, [19] for a related result in the Brownian case). However, in many applications, the automatic semimartingale property is especially useful, as it allows us to perform so-called "verification" directly and without additional assumptions or the invocation of the dynamic-programming principle.

The way we overcame these difficulties in the present paper is by: (1) approximating our system by a sequence of well-behaved systems, (2) combining analytic methods described above with probabilistic ones to obtain adequate uniform estimates on these approximations and (3) showing that the passage to the limit preserves the semimartingale property (as well as the equation itself), while relying mostly on probabilistic arguments. This way, we obtain a solution of the form $\boldsymbol{Y}_{t}=\boldsymbol{v}\left(t, X_{t}\right), \boldsymbol{Z}_{t}=\boldsymbol{w}\left(t, X_{t}\right)$, where $\boldsymbol{v}$ is locally Hölder continuous, $\boldsymbol{v}\left(t, X_{t}\right)$ is a semimartingale, and $\boldsymbol{w}$ is the weak Jacobian of $\boldsymbol{v}$. This strategy bypasses regularity and pointwise estimates on $\boldsymbol{w}$, which is typically needed to establish a PDE solution in more analytical approaches. While we are still far from completely understanding of the appropriate class of functions to replace viscosity solutions in the multidimensional case, we feel that the functions with above properties constitute a promising first step.

1.3. Our results-Sufficient conditions and examples. As a complement to our main existence/uniqueness theorem, we provide a sufficient condition for the existence of Lyapunov functions-termed the Bensoussan-Frehse (BF) conditionas well as a somewhat simpler sufficient condition for a priori boundedness. The (BF) condition, a list of algebraic conditions on various terms in the generator, is a slight generalization of the structural condition discovered by Bensoussan and Frehse in [7]. We add a term of sub-quadratic growth and also allow for a small "error" around the structure, thus incorporating both "smallness" and Bensoussan and Frehse's structural condition into a single condition. Generators in many solvable Markovian BSDE systems described in the prior literature satisfy our condition (BF). Nevertheless, (BF) may not be suitable for our Example 3.2 below where the Lyapunov function is constructed by geometric argument.

We illustrate our main results and the sufficient conditions with a number of examples. Our first example shows that the stochastic equilibria exist and are unique in a class of incomplete financial market models, with heterogeneous "exponential" agents. Next, we construct a class of martingales on differential manifolds with connections under fewer assumptions than before. Then, we treat two nonzero sum stochastic games, namely, a game with "cooperation and hindrance," and a risk-sensitive stochastic differential game; we show that Nash equilibria exist in both. Our final example focuses on a different aspect of our results and treats a one-dimensional equation.

1.4. Structure of the paper. After this Introduction, we describe the setting and state our main theorem and various sufficient conditions for its assumptions in Section 2. Section 3 contains examples, while the proofs are divided between two sections: Section 4 deals with Hölder boundedness and contains the bulk of the 
partial-regularity and hole-filling estimates, while all the other proofs are collected in Section 5.

1.5. Notation and conventions. For a scalar function $u, D u$ denotes its $\left(\mathbb{R}^{d}\right.$ valued) spatial gradient, interpreted as a row vector, while $D^{2} u$ denotes its (spatial) Hessian matrix. Individual spatial partial derivatives of are denoted by subscripts, that is, $D_{i} u=\frac{\partial}{\partial x^{i}} u$ and $D_{i j} u$ stands for $\frac{\partial^{2}}{\partial x^{i} \partial x^{j}} u$. In the vectorial ( $\mathbb{R}^{N}$-valued) case-which we mark by bold symbols- $D \boldsymbol{u}$ is understood as the $\mathbb{R}^{N \times d}$-Jacobian matrix.

We will also have occasion to evaluate bilinear forms on function gradients; for a $d \times d$ matrix $S$ we write $\langle D u, D w\rangle_{S}=\sum_{i j} D_{i} u D_{j} w S_{i j}=D u S D w^{\top}$. More generally, for a square matrix $S$ and two matrices $A$ and $B$ of appropriate dimensions, we write $\langle A, B\rangle_{S}$ for the matrix $A S B^{\top}$. The Frobenius product of matrices is denoted by $A: B$, that is, for square matrices $A$ and $B$ of the same dimension, we define $A: B=\sum_{i j} A_{i j} B_{i j}=\operatorname{Tr}\left(A^{\top} B\right)$. The Frobenius norm of a square matrix $A$ is given by $\sqrt{A: A}$.

The notation $|\cdot|$ is used both for the Lebesgue measure of a subset of $\mathbb{R}^{d}$, as well as for the Euclidean norm in any Euclidean space; it should be interpreted as the Frobenius norm, in case its argument is a matrix. For $(t, x) \in \mathbb{R} \times \mathbb{R}^{d}$, we use an anisotropic norm, namely, we set $|(t, x)|=\max (\sqrt{|t|},|x|)$. The closed ball of radius $R$ around $x$ in $\mathbb{R}^{d}$ is denoted by $B_{R}(x)$. In the special case when $x=0$ and $R=n \in \mathbb{N}$, we use simply $B_{n}$.

The notation $\|\cdot\|$ will be reserved for infinite-dimensional spaces. More specifically, unless defined otherwise, $\|\cdot\|$ stands for the $\mathbb{L}^{2}$-norm, both on the underlying probability space, and on an appropriate domain.

For $r \in \mathbb{N}$ and a (generally matrix-valued) process $\left(\boldsymbol{Z}_{u}\right)_{u \in[t, T]}$, we write $\boldsymbol{Z} \in \mathcal{P}^{r}$ if $\int_{t}^{T}\left|\boldsymbol{Z}_{u}\right|^{r} d u<\infty$, a.s. The stochastic integral $\int \boldsymbol{Z}_{s} d W_{s}$ of $\boldsymbol{Z} \in \mathcal{P}^{2}$ with respect to a vector Brownian motion $W$, defines a vector-valued process whose $i$ th component is given by $\sum_{j} \int \boldsymbol{Z}_{s}^{i j} d W_{s}^{j}$. We write $\boldsymbol{Z} \in$ bmo if $\sup _{\tau \in \mathcal{T}}\left\|\mathbb{E}_{\tau}\left[\int_{\tau}^{T}\left|Z_{u}\right|^{2} d u\right]\right\|_{\mathbb{L}^{\infty}}<\infty$, where $\mathcal{T}$ is the set of [0,T]-valued stopping times and $\mathbb{E}_{\tau}$ denotes the conditional expectation $\mathbb{E}\left[\cdot \mid \mathcal{F}_{\tau}\right]$ with respect to $\mathcal{F}_{\tau}$. The notation $d F \stackrel{m}{=} \alpha$ means $F-\int_{0}^{\cdot} \alpha_{s} d s$ is a local martingale. Standard localization techniques and boundedness of processes involved can be used to show that all local martingales in the sequel can be treated as martingales effectively, therefore, we will treat them as such without explicit mention.

For $\alpha \in(0,1]$, a compact subset $K$ of $\mathbb{R}^{d}$ and a function $\boldsymbol{v}:[0, T] \times \mathbb{R}^{d} \rightarrow \mathbb{R}^{N}$, the Hölder seminorm $[\boldsymbol{v}]_{\alpha, K}$ is defined by

$$
[\boldsymbol{v}]_{\alpha, K}=\sup _{\left(t^{\prime}, x^{\prime}\right) \neq(t, x) \in[0, T] \times K} \frac{\left|\boldsymbol{v}\left(t^{\prime}, x^{\prime}\right)-\boldsymbol{v}(t, x)\right|}{\left|(t, x)-\left(t^{\prime}, x^{\prime}\right)\right|^{\alpha}} .
$$

Sequences are denoted by curly brackets $\{\cdot\}$. The index $n \in \mathbb{N}$ or $m \in \mathbb{N}$ is usually omitted and will always be clear from the context. 


\section{Main results.}

\subsection{The setup, standing assumptions and key concepts.}

2.1.1. The driving diffusion. We work on a probability space $\left(\Omega, \mathcal{F}_{T}, \mathbb{P}\right)$, on which a $d$-dimensional Brownian motion $\left(W_{t}\right)_{t \in[0, T]}$ is defined. With $\mathbb{F}=\left(\mathcal{F}_{t}\right)$ denoting the augmented filtration generated by $W$, we consider the stochastic differential equation:

$$
d X_{t}=b\left(t, X_{t}\right) d t+\sigma\left(t, X_{t}\right) d W_{t}
$$

where:

1. the drift vector $b:[0, T] \times \mathbb{R}^{d} \rightarrow \mathbb{R}^{d}$ is uniformly bounded,

2. the dispersion matrix $\sigma:[0, T] \times \mathbb{R}^{d} \rightarrow \mathbb{R}^{d \times d}$ is symmetric and there exist a constant $\Lambda>0$ such that $\Lambda|z|^{2} \geq|z \sigma(t, x)|^{2} \geq \frac{1}{\Lambda}|z|^{2}$, for all $(t, x) \in[0, T] \times \mathbb{R}^{d}$ and all $z \in \mathbb{R}^{d}$, and

3. there exists a constant $L$ such that, for all $t \in[0, T], x, x^{\prime} \in \mathbb{R}^{d}$, we have

$$
\left|b(t, x)-b\left(t, x^{\prime}\right)\right|+\left|\sigma(t, x)-\sigma\left(t, x^{\prime}\right)\right| \leq L\left|x-x^{\prime}\right| .
$$

These conditions ensure, in particular, that for each $(t, x) \in[0, T] \times \mathbb{R}^{d}$, there exists a unique strong solution $\left(X_{u}^{t, x}\right)_{u \in[t, T]}$ of (2.1), defined on $[t, T]$, such that $X_{t}^{t, x}=x$. For notational reasons, we extend $X^{t, x}$ by setting $X_{u}^{t, x}=x$, for $u \in[0, t)$, and denote by $\mathbb{P}^{t, x}$ its law on the canonical space $C^{d}[0, T]$.

2.1.2. Markovian and Hölderian solutions. Given $b_{0} \in \mathbb{R}^{d}$ and a sequence $\left\{\alpha_{n}\right\}$ in $(0,1]$, a sequence $\left\{\boldsymbol{v}^{m}\right\}$ is said to be bounded in $C_{\mathrm{loc}, b_{0}}^{\left\{\alpha_{n}\right\}}\left([0, T] \times \mathbb{R}^{d}\right)$ if there exists a sequence $\left\{c_{n}\right\}$ of positive constants, such that, for all $m, n \in \mathbb{N}$,

$$
\left\|\boldsymbol{v}^{m}\right\|_{C^{\alpha_{n}\left([0, T] \times B_{n}\left(b_{0}\right)\right)}}=\left\|\boldsymbol{v}^{m}\right\|_{\mathbb{L}^{\infty}\left([0, T] \times B_{n}\left(b_{0}\right)\right)}+\left[\boldsymbol{v}^{m}\right]_{\alpha_{n}, B_{n}\left(b_{0}\right)} \leq c_{n} .
$$

We write $\boldsymbol{v} \in C_{\mathrm{loc}, b_{0}}^{\left\{\alpha_{n}\right\}}$ if the constant sequence $\{\boldsymbol{v}\}$ is bounded in $C_{\mathrm{loc}, b_{0}}^{\left\{\alpha_{n}\right\}}$. If the sequence $\left\{c_{n}\right\}$ is uniform for all $b_{0} \in \mathbb{R}^{d}$, we say $\boldsymbol{v} \in C_{\mathrm{loc}}^{\left\{\alpha_{n}\right\}}$. A completely analogous construction yields the family of local Hölder spaces $C_{\text {loc, } b_{0}}^{\left\{\alpha_{n}\right\}}\left(\mathbb{R}^{d}\right)$ and $C_{\text {loc }}^{\left\{\alpha_{n}\right\}}$ over $\mathbb{R}^{d}$ instead of $[0, T] \times \mathbb{R}^{d}$. Various spaces of continuously (nonfractionally) differentiable functions are defined in the standard manner.

DEFinition 2.1 (A Markovian solution to BSDE). Given Borel functions $\boldsymbol{f}:[0, T] \times \mathbb{R}^{d} \times \mathbb{R}^{N} \times \mathbb{R}^{N \times d} \rightarrow \mathbb{R}^{N}$ and $\boldsymbol{g}: \mathbb{R}^{d} \rightarrow \mathbb{R}^{N}$, a pair $(\boldsymbol{v}, \boldsymbol{w})$ of Borel functions with the domain $[0, T] \times \mathbb{R}^{d}$ and co-domains $\mathbb{R}^{N}$ and $\mathbb{R}^{N \times d}$, respectively, is a called a Markovian solution to the system

$$
d \boldsymbol{Y}_{t}=-\boldsymbol{f}\left(t, X_{t}, \boldsymbol{Y}_{t}, \boldsymbol{Z}_{t}\right) d t+\boldsymbol{Z}_{t} \sigma\left(t, X_{t}\right) d W_{t}, \quad \boldsymbol{Y}_{T}=\boldsymbol{g}\left(X_{T}\right),
$$

of backward stochastic differential equations if, for all $(t, x) \in[0, T] \times \mathbb{R}^{d}$, 
1. $\boldsymbol{Y}^{t, x}:=\boldsymbol{v}\left(\cdot, X^{t, x}\right)$ is a continuous process, $\boldsymbol{Z}^{t, x}:=\boldsymbol{w}\left(\cdot, X^{t, x}\right) \in \mathcal{P}^{2}$, and $\boldsymbol{f}\left(\cdot, X^{t, x}, \boldsymbol{Y}^{t, x}, \boldsymbol{Z}^{t, x}\right) \in \mathcal{P}^{1}$,

2. for all $t^{\prime} \in[t, T]$, we have

$$
\begin{aligned}
\boldsymbol{Y}_{t^{\prime}}^{t, x}= & \boldsymbol{g}\left(X_{T}^{t, x}\right) \\
& +\int_{t^{\prime}}^{T} \boldsymbol{f}\left(u, X_{u}^{t, x}, \boldsymbol{Y}_{u}^{t, x}, \boldsymbol{Z}_{u}^{t, x}\right) d u-\int_{t^{\prime}}^{T} \boldsymbol{Z}_{u}^{t, x} \sigma\left(u, X_{u}^{t, x}\right) d W_{u},
\end{aligned}
$$

A Markovian solution $(\boldsymbol{v}, \boldsymbol{w})$ to $(2.2)$ is said to be bounded if $\boldsymbol{v}$ is bounded, continuous if $\boldsymbol{v}$ is continuous, locally Hölderian if $\boldsymbol{v} \in C_{\mathrm{loc}, b_{0}}^{\left\{\alpha_{n}\right\}}$, for some $b_{0} \in \mathbb{R}^{d}$ and some sequence $\left\{\alpha_{n}\right\}$ in $(0,1]$, and a bmo-solution if $\boldsymbol{Z}^{t, x} \in$ bmo for all $(t, x) \in[0, T] \times \mathbb{R}^{d}$.

REMARK 2.2. For Markovian BSDE, it is customary to consider the generator $\tilde{\boldsymbol{f}}(t, x, \boldsymbol{y}, \boldsymbol{z} \sigma)$ instead of our $\boldsymbol{f}(t, x, \boldsymbol{y}, \boldsymbol{z})$. Due to our assumptions on $\sigma$, these are equivalent and we maintain the generator as $f$ for notational convenience later on.

2.1.3. Lyapunov functions. The key condition in our main result below concerns the existence of sequence of functions which we term the Lyapunov functions. We abbreviate $a=\sigma \sigma^{\top}$ and define $\langle z, z\rangle_{a(t, x)}=\boldsymbol{z} \sigma(t, x)(z \sigma(t, x))^{\top}$.

Definition 2.3 (Lyapunov functions). Let $\boldsymbol{f}:[0, T] \times \mathbb{R}^{d} \times \mathbb{R}^{N} \times \mathbb{R}^{N \times d} \rightarrow$ $\mathbb{R}^{N}$ be a Borel function and let $c>0$ be a constant. A pair $(h, k)$ of nonnegative functions, with $h \in C^{2}\left(\mathbb{R}^{N}\right)$ and $k$ Borel, is said to be a $c$-Lyapunov pair for $f$ if $h(\mathbf{0})=0, D h(\mathbf{0})=\mathbf{0}$, and

$$
\frac{1}{2} D^{2} h(\boldsymbol{y}):\langle z, z\rangle_{a(t, x)}-D h(\boldsymbol{y}) \boldsymbol{f}(t, x, \boldsymbol{y}, \boldsymbol{z}) \geq|z|^{2}-k(t, x),
$$

for all $(t, x, \boldsymbol{y}, \boldsymbol{z}) \in[0, T] \times \mathbb{R}^{d} \times \mathbb{R}^{N} \times \mathbb{R}^{N \times d}$, with $|\boldsymbol{y}| \leq c$. We write $(h, k) \in$ $\mathbf{L y}(\boldsymbol{f}, c)$.

Given $b_{0} \in \mathbb{R}^{d}$ and a sequence $\left\{c_{n}\right\}$ of positive constants, a pair $\left(\left\{h_{n}\right\},\left\{k_{n}\right\}\right)$ of sequences of nonnegative functions, with $h_{n} \in C^{2}\left(\mathbb{R}^{N}\right)$ and $k_{n}$ Borel, is called a local $\left\{c_{n}\right\}$-Lyapunov pair for $\boldsymbol{f}$, if $h_{n}(\mathbf{0})=0, D h_{n}(\mathbf{0})=\mathbf{0}$ and

$$
\frac{1}{2} D^{2} h_{n}(\boldsymbol{y}):\langle\boldsymbol{z}, \boldsymbol{z}\rangle_{a(t, x)}-D h_{n}(\boldsymbol{y}) \boldsymbol{f}(t, x, \boldsymbol{y}, \boldsymbol{z}) \geq|\boldsymbol{z}|^{2}-k_{n}(t, x),
$$

for all $n \in \mathbb{N},(t, x, \boldsymbol{y}, \boldsymbol{z}) \in[0, T] \times B_{n}\left(b_{0}\right) \times \mathbb{R}^{N} \times \mathbb{R}^{N \times d}$, with $|\boldsymbol{y}| \leq c_{n}$. We write $\left(\left\{h_{n}\right\},\left\{k_{n}\right\}\right) \in \mathbf{L y}_{\text {loc }}\left(\boldsymbol{f},\left\{c_{n}\right\}\right)$.

\section{REMARK 2.4 .}

1. Suppose that the process $\boldsymbol{Y}$ has a semimartingale decomposition as in (2.2) (i.e., solves the BSDE system) and satisfies the bound $|\boldsymbol{Y}| \leq c$. A func- 
tion $h$ for which (2.3) holds has the property that $h\left(\boldsymbol{Y}_{t}\right)$ is a semimartingale with the finite variation part dominating (in the increasing order) the process $\int_{0}^{\cdot}\left(\left|Z_{u}\right|^{2}-k\left(u, X_{u}\right)\right) d u$. The function $k$ will often be constant, but certain applications require more flexibility. If one wants to deal with unbounded $\boldsymbol{Y}$, a layer of localization-expressed through the dependence on $n$ and the sequence $\left\{c_{n}\right\}$ in the local version-is necessary.

2. It is interesting to note that in the scalar case $(N=1)$, and when the generator $\boldsymbol{f}$ grows at most quadratically in $z$, it is essentially sufficient to look for Lyapunov pairs with $h(y)=\exp (\alpha y)$, for large enough $\alpha$. As we shall see below, this no longer works in the vector case, which leads to nontrivial constructions of Lyapunov pairs under specific structural conditions.

3. Let $(\boldsymbol{v}, \boldsymbol{w})$ be a bounded solution to (2.2) whose generator $\boldsymbol{f}$ admits a $\|\boldsymbol{v}\|_{\mathbb{L}^{\infty}}$ Lyapunov pair $(h, k)$ with $k$ bounded. Item (1), together with boundedness of $\boldsymbol{v}$ and $k$, implies that $Z=\boldsymbol{w}(\cdot, X) \in$ bmo. Hence, $(\boldsymbol{v}, \boldsymbol{w})$ is a bmo-solution.

2.2. A uniform estimate. The first main result of the paper, contained in Theorem 2.5 below, provides an abstract stencil for a uniform estimate for a family of BSDE systems under several assumptions, most notable of which is the existence of a Lyapunov pair, uniform for all systems in the family. Sufficient conditions for these assumptions and examples will be given shortly.

THEOREM 2.5 (Uniform estimate). Let $\left\{\boldsymbol{f}^{m}\right\}$ and $\left\{\boldsymbol{g}^{m}\right\}$ be sequences of Borel functions $\boldsymbol{f}^{m}:[0, T] \times \mathbb{R}^{d} \times \mathbb{R}^{N} \times \mathbb{R}^{N \times d} \rightarrow \mathbb{R}^{N}$ and $\boldsymbol{g}^{m}: \mathbb{R}^{d} \rightarrow \mathbb{R}^{N}$ such that, for each $m \in \mathbb{N}$, the BSDE system

$$
d \boldsymbol{Y}_{t}=-\boldsymbol{f}^{m}\left(t, X_{t}, \boldsymbol{Y}_{t}, \boldsymbol{Z}_{t}\right) d t+\boldsymbol{Z}_{t} \sigma\left(t, X_{t}\right) d W_{t}, \quad \boldsymbol{Y}_{T}=\boldsymbol{g}^{m}\left(X_{T}\right),
$$

admits a Markovian solution $\left(\boldsymbol{v}^{m}, \boldsymbol{w}^{m}\right)$.

Suppose that there exist $b_{0} \in \mathbb{R}^{d}$ and sequences $\left\{M_{n}\right\},\left\{c_{n}\right\}$ in $[0, \infty),\left\{\alpha_{n}\right\}$ in $(0,1]$ and $\left\{q_{n}\right\}$ with $q_{n}>1+d / 2$, such that:

1. ( $C_{\mathrm{loc}}^{\alpha}$-regularity of the terminal condition) The sequence $\left\{\boldsymbol{g}^{m}\right\}$ is bounded in $C_{\text {loc }, b_{0}}^{\left\{\alpha_{n}\right\}}$

2. (A priori continuity and local uniform boundedness) For all $m, n \in \mathbb{N}, \boldsymbol{v}^{m}$ is continuous on $[0, T] \times \mathbb{R}^{d}$ and

$$
\left|\boldsymbol{v}^{m}(t, x)\right| \leq c_{n} \quad \text { for all }(t, x) \in[0, T] \times B_{n}\left(b_{0}\right) .
$$

3. (Local uniform quadratic growth) For each $n \in \mathbb{N}$, there exist functions $\left\{k_{n}^{m}\right\}$ such that

$$
\sup _{m \in \mathbb{N}}\left\|k_{n}^{m}\right\|_{\mathbb{L}^{q_{n}}\left([0, T] \times B_{n}\left(b_{0}\right)\right)}<\infty \quad \text { and } \quad\left|\boldsymbol{f}^{m}(t, x, \boldsymbol{y}, \boldsymbol{z})\right| \leq M_{n}\left(|\boldsymbol{z}|^{2}+k_{n}^{m}(t, x)\right),
$$

for all $m \in \mathbb{N},(t, x) \in[0, T] \times B_{n}\left(b_{0}\right),|\boldsymbol{y}| \leq c_{n}$, and $z \in \mathbb{R}^{N \times d}$. 
4. (Local Lyapunov pair) There exist functions $\left\{h_{n}\right\}$ such that $\left(\left\{h_{n}\right\},\left\{k_{n}^{m}\right\}\right)$ is a local $\left\{2 c_{n}\right\}$-Lyapunov pair for $\boldsymbol{f}^{m}$ for each $m \in \mathbb{N}$, that is, $\left(\left\{h_{n}\right\},\left\{k_{n}^{m}\right\}\right) \in$ $\mathbf{L y}_{\text {loc }}\left(\boldsymbol{f}^{m},\left\{2 c_{n}\right\}\right)$.

Then the sequence $\left\{\boldsymbol{v}^{m}\right\}$ is bounded in $C_{\mathrm{loc}, b_{0}}^{\left\{\alpha_{0}^{\prime}\right\}}$, for some $\left\{\alpha_{n}^{\prime}\right\}$ in $(0,1]$. Moreover, for each $n$, the Hölder seminorm $\left[\boldsymbol{v}^{m}\right] \alpha_{n}^{\prime}, B_{n}\left(b_{0}\right)$ depends only on $d, N, T, \Lambda, L$,

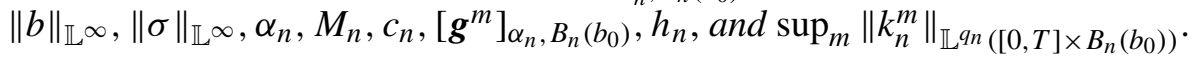

\section{REMARK 2.6.}

1. The sequence $\left\{h_{n}\right\}$ in condition (4) above is chosen uniformly for all $\left\{\boldsymbol{f}^{m}\right\}$. Therefore, the inequality (2.4) is satisfied for all $\boldsymbol{f}^{m}$ and $k_{n}^{m}$. It is without loss of generality to have $|z|^{2}$ on the right-hand side of (2.4), since any positive constant $\delta_{n}$ before $|z|^{2}$ can be normalized to 1 after scaling $h_{n}$ and $k_{n}^{m}$ by $1 / \delta_{n}$.

2. Applying Theorem 2.5 to a constant sequence (i.e., all $\left\{\boldsymbol{f}^{m}\right\}$ and $\left\{\boldsymbol{g}^{m}\right\}$ are the same for different $m$ ), we obtain an a priori estimate for a continuous Markovian solution of a single system: let $(\boldsymbol{v}, \boldsymbol{w})$ be a continuous Markovian solution (2.2) whose data $\boldsymbol{f}$ and $\boldsymbol{g}$ satisfy assumptions in Theorem 2.5, then $\boldsymbol{v}$ is locally Hölderian, that is, $\boldsymbol{v} \in C_{\text {loc }, b_{0}}^{\left\{\alpha_{n}\right\}}$.

One of the advantages of our probabilistic approach is that the uniform Hölder bound on $\left\{\boldsymbol{v}^{m}\right\}$ in Theorem 2.5 is sufficient to establish the existence result in Theorem 2.8 below. To make a connection with a typical analytic treatment of related PDEs, where regularity and bounds of $\boldsymbol{w}$ need to be obtained, we provide some pertinent information in the following remark.

\section{REMARK 2.7.}

1. Without structural conditions on $\boldsymbol{f}$, uniform $\mathbb{L}^{\infty}$-bounds for systems do not always lead to gradient bounds, as evidenced by the following example due to E. Heinz. Consider the following quadratic system of PDE:

$$
v_{t}^{i}-v_{x x}^{i}=v^{1}\left(\left(v_{x}^{1}\right)^{2}+\left(v_{x}^{2}\right)^{2}\right), \quad i=1,2 .
$$

For any $m \in \mathbb{N}, v^{1}=\cos (m x)$ and $v^{2}=\sin (m x)$ is a (stationary) solution, but clearly, $\|\nabla v\|_{\mathbb{L}^{\infty}}=m$ cannot be controlled by $\|v\|_{\mathbb{L}^{\infty}}=1$ (and a universal constant independent of $m$ ). For a general system of the form

$$
\partial_{t} \boldsymbol{v}-\frac{1}{2} \Delta \boldsymbol{v}+\boldsymbol{f}(t, x, \boldsymbol{v}, \nabla \boldsymbol{v})=0,
$$

a local estimate of $\|\nabla v\|_{\mathbb{L}^{\infty}}$ is established in [44], Theorem 6.1, in the case when $f$ satisfies a condition of the form

$$
|\boldsymbol{f}(t, x, \boldsymbol{v}, \boldsymbol{p})| \leq[\varepsilon|\boldsymbol{v}|+P(|\boldsymbol{p}|,|\boldsymbol{v}|)](1+|\boldsymbol{p}|)^{2},
$$


for some sufficiently small $\varepsilon>0$ and $P(|p|,|v|) \rightarrow 0$ as $|p| \rightarrow \infty$. When $f$ has at most linear growth in $p$, the same local estimate is established in [23] using a probabilistic techniques.

2. When $\boldsymbol{v}$ is Hölder continuous and there exists $k_{n} \in \mathbb{L}^{q}\left([0, T] \times B_{n}\left(b_{0}\right)\right)$ with $q>1+d / 2$ such that

$$
|\boldsymbol{f}(t, x, \boldsymbol{y}, \boldsymbol{z})| \leq M_{n}\left(|z|^{2}+k_{n}(t, x)\right),
$$

for all $(t, x) \in[0, T] \times B_{n}\left(b_{0}\right), y \in \mathbb{R}^{N}$, and $z \in \mathbb{R}^{N \times d}$, then [7], Proposition 5.1, used regularity theory of elliptic systems in [31] to show that $\boldsymbol{v} \in W_{q}^{2,1}([0, T] \times$ $\left.B_{n}\left(b_{0}\right)\right)$. In particular, when $q>2+d$, Sobolev embedding Theorem (see [44], Lemma 3.3) implies that $\boldsymbol{w}$, as the weak Jacobian of $\boldsymbol{v}$, is Hölder continuous on $[0, T] \times B_{n}\left(b_{0}\right)$.

2.3. Existence and uniqueness. A direct consequence of the uniform estimate in Theorem 2.5 is the existence of a Markovian solution to the system (2.7), whose data $(\boldsymbol{f}, \boldsymbol{g})$ are approximated by a sequence $\left\{\boldsymbol{f}^{m}, \boldsymbol{g}^{m}\right\}$.

THEOREM 2.8 (Existence by approximation). Let $\boldsymbol{f}:[0, T] \times \mathbb{R}^{d} \times \mathbb{R}^{N} \times$ $\mathbb{R}^{N \times d} \rightarrow \mathbb{R}^{N}$ and $\boldsymbol{g}: \mathbb{R}^{d} \rightarrow \mathbb{R}$ be a pair of Borel functions. Assume that there exist sequences $\left\{\boldsymbol{f}^{m}\right\}$ and $\left\{\boldsymbol{g}^{m}\right\}$ which satisfy the assumptions of Theorem 2.5 and

$$
\lim _{m \rightarrow \infty} \boldsymbol{f}^{m}\left(t, x, \boldsymbol{y}^{m}, \boldsymbol{z}^{m}\right)=\boldsymbol{f}(t, x, \boldsymbol{y}, \boldsymbol{z}) \quad \text { and } \lim _{m \rightarrow \infty} \boldsymbol{g}^{m}(x)=\boldsymbol{g}(x),
$$

for all $(t, x, y, z) \in[0, T] \times \mathbb{R}^{d} \times \mathbb{R}^{N} \times \mathbb{R}^{N \times d}$ and all sequences $\boldsymbol{y}^{m} \rightarrow \boldsymbol{y}$ and $z^{m} \rightarrow z$. Then the system

$$
d \boldsymbol{Y}_{t}=-\boldsymbol{f}\left(t, X_{t}, \boldsymbol{Y}_{t}, \boldsymbol{Z}_{t}\right) d t+\boldsymbol{Z}_{t} \sigma\left(t, X_{t}\right) d W_{t}, \quad \boldsymbol{Y}_{T}=\boldsymbol{g}\left(X_{T}\right),
$$

admits a locally Hölderian solution $(\boldsymbol{v}, \boldsymbol{w})$ such that $\boldsymbol{v}$ is a locally uniform limit of a subsequence of $\left\{\boldsymbol{v}^{m}\right\}$ in Theorem 2.5, and $\boldsymbol{w}$ is the weak Jacobian of $\boldsymbol{v}$ on $(0, T) \times \mathbb{R}^{d}$.

The solutions produced in Theorem 2.8 are not necessarily unique, even when the solutions to the approximating equations are. Indeed, one only needs to consider the case where $\boldsymbol{f} \equiv 0$ and where $\left\{\boldsymbol{g}^{m}\right\}$ is a sequence of bounded and smooth approximations to the function $\boldsymbol{g}$ appearing in Tychonov's nonuniqueness theorem (see [39], page 171) for the heat equation.

As we shall see below, these pathologies disappear under appropriate conditions on $\boldsymbol{f}$ and $\boldsymbol{g}$. When the Hölder norm of $\boldsymbol{g}$ does not depend on $b_{0}, \boldsymbol{f}$ does not depend on $\boldsymbol{y}$ and satisfies additional regularity assumption in $z$, uniqueness is recovered. Two Markovian solutions, $(\boldsymbol{v}, \boldsymbol{w})$ and $\left(\boldsymbol{v}^{\prime}, \boldsymbol{w}^{\prime}\right)$, are considered equal if $\boldsymbol{v}(t, x)=$ $\boldsymbol{v}^{\prime}(t, x)$ for all $(t, x) \in[0, T] \times \mathbb{R}^{d}$ and $\boldsymbol{w}=\boldsymbol{w}^{\prime}$, a.e., with respect to the Lebesgue measure on $[0, T] \times \mathbb{R}^{d}$.

THEOREM 2.9 (Uniqueness). Suppose that:

1. $\boldsymbol{g} \in C_{\mathrm{loc}}^{\left\{\alpha_{n}\right\}} \cap \mathbb{L}^{\infty}$ for some sequence $\left\{\alpha_{n}\right\}$ in $(0,1]$; 
2. $\boldsymbol{f}$ is continuous, does not depend on $\boldsymbol{y}$, and there exists $M \geq 0$ such that $|\boldsymbol{f}(t, x, z)| \leq M\left(1+|z|^{2}\right) \quad$ and $\quad\left|\boldsymbol{f}(t, x, z)-\boldsymbol{f}\left(t, x, \boldsymbol{z}^{\prime}\right)\right| \leq M\left(|z|+\left|\boldsymbol{z}^{\prime}\right|\right)\left|\boldsymbol{z}-\boldsymbol{z}^{\prime}\right|$, for all $(t, x) \in[0, T] \times \mathbb{R}^{d}, z, z^{\prime} \in \mathbb{R}^{d \times N}$;

3. there exists a (global) Lyapunov pair $(h, k) \in \mathbf{L y}(\boldsymbol{f}, c)$ with $k$ bounded for some $c>0$.

Then (2.2) admits at most one continuous solution $(\boldsymbol{v}, \boldsymbol{w})$ with $\|\boldsymbol{v}\|_{\mathbb{L}^{\infty}} \leq c$.

2.4. A sufficient condition for existence and uniqueness. This section provides explicit conditions on the generator $\boldsymbol{f}$ and the terminal condition $\boldsymbol{g}$ such that assumptions in Theorems 2.5, 2.8 and 2.9 hold for (2.2). While the proof depends on the abstract Theorem 2.5 above, we state it in a self-contained form to make it more accessible to a reader interested in its applications.

We start with a structural condition on the generator $f$. To the best of our knowledge, a version of it was first formulated in [7]. We present here a generalization including a subquadratic term; a further generalization will be discussed in Remark 2.15 below. We interpret $z \in \mathbb{R}^{N \times d}$ as an $N \times d$-matrix, and use $z^{j}$ to denote its $j$ th row, $j=1, \ldots, N$. In the vector case, the superscript $j$ denotes the $j$ th component.

DEFINITION 2.10 [The Bensoussan-Frehse (BF) condition]. We say that a continuous function $\boldsymbol{f}:[0, T] \times \mathbb{R}^{d} \times \mathbb{R}^{N} \times \mathbb{R}^{N \times d} \rightarrow \mathbb{R}^{N}$ satisfies the condition $(B F)$ if it admits a decomposition of the form

$$
\boldsymbol{f}(t, x, \boldsymbol{y}, \boldsymbol{z})=\operatorname{diag}(z \mathbf{I}(t, x, \boldsymbol{y}, \boldsymbol{z}))+\mathbf{q}(t, x, \boldsymbol{y}, \boldsymbol{z})+\mathbf{s}(t, x, \boldsymbol{y}, \boldsymbol{z})+\mathbf{k}(t, x),
$$

such that the functions I : $[0, T] \times \mathbb{R}^{d} \times \mathbb{R}^{N} \times \mathbb{R}^{N \times d} \rightarrow \mathbb{R}^{d \times N}$ and $\mathbf{q}, \mathbf{s}, \mathbf{k}:[0, T] \times$ $\mathbb{R}^{d} \times \mathbb{R}^{N} \times \mathbb{R}^{N \times d} \rightarrow \mathbb{R}^{N}$ have following property: there exist $b_{0} \in \mathbb{R}^{d}$ and two sequences $\left\{C_{n}\right\}$ and $\left\{q_{n}\right\}$ of positive constants with $q_{n}>1+d / 2$, and a sequence $\left\{\kappa_{n}\right\}$ of functions $\kappa_{n}:[0, \infty) \rightarrow[0, \infty)$ with $\lim _{w \rightarrow \infty} \kappa_{n}(w) / w^{2}=0$ such that, for each $n \in \mathbb{N}$ and all $(t, x, \boldsymbol{y}, \boldsymbol{z}) \in[0, T] \times B_{n}\left(b_{0}\right) \times \mathbb{R}^{N} \times \mathbb{R}^{N \times d}$ we have

$|\mathbf{I}(t, x, \boldsymbol{y}, z)| \leq C_{n}(1+|z|)$,

$\left|\mathbf{q}^{i}(t, x, \boldsymbol{y}, \boldsymbol{z})\right| \leq C_{n}\left(1+\sum_{j=1}^{i}\left|z^{j}\right|^{2}\right), \quad i=1, \ldots, N, \quad$ (quadratic-triangular)

$|\mathbf{s}(t, x, \boldsymbol{y}, \boldsymbol{z})| \leq \kappa_{n}(|z|)$,

$\mathbf{k} \in \mathbb{L}^{q_{n}}\left([0, T] \times B_{n}\right)$, (quadratic-linear)

(subquadratic)

( $z$-independent)

In that case, we write $\boldsymbol{f} \in \mathbf{B F}\left(\left\{C_{n}\right\},\left\{\kappa_{n}\right\},\left\{q_{n}\right\}\right)$.

The (BF) conditions are simple enough to be easily checked in applications, but also strong enough to yield the following result which will play a major role in the existence theorem below. 
PROPOSITION 2.11 [Existence of Lyapunov pairs under condition (BF)]. Let $\left\{c_{n}\right\}$ is an arbitrary sequence of positive constants, and $\boldsymbol{f}$ a function in $\mathbf{B F}\left(\left\{C_{n}\right\},\left\{\kappa_{n}\right\},\left\{q_{n}\right\}\right) z$ :

1. There exists a local $\left\{c_{n}\right\}$-Lyapunov pair $\left(\left\{h_{n}\right\},\left\{k_{n}\right\}\right)$ for $\boldsymbol{f}$. Furthermore, the same pair $\left(\left\{h_{n}\right\},\left\{k_{n}\right\}\right)$ is a local $\left\{c_{n}\right\}$-Lyapunov pair for any other function $\boldsymbol{f}^{\prime} \in$ $\mathbf{B F}\left(\left\{C_{n}\right\},\left\{\kappa_{n}\right\},\left\{q_{n}\right\}\right)$.

2. If, additionally, the sequences $\left\{C_{n}\right\},\left\{q_{n}\right\}$ and $\left\{\kappa_{n}\right\}$ are constant (in $\left.n\right)$, then, for each c, a (global) c-Lyapunov pair for $\boldsymbol{f}$ exists.

Another ingredient necessary to guarantee the existence of a solution to (2.2) is a priori boundedness. We remind the reader that a set of nonzero vectors $\boldsymbol{a}_{1}, \ldots, \boldsymbol{a}_{K}$ in $\mathbb{R}^{N}$ (with $K>N$ ) is said to positively span $\mathbb{R}^{N}$, if, for each $\boldsymbol{a} \in \mathbb{R}^{N}$ there exist nonnegative constants $\lambda_{1}, \ldots, \lambda_{K}$ such that

$$
\lambda_{1} a_{1}+\cdots+\lambda_{K} a_{K}=a .
$$

The following two well-known characterizations (see [22]), presented here for reader's convenience, make positively-spanning sets easy to spot: (1) Nonzero vectors $\boldsymbol{a}_{1}, \ldots, \boldsymbol{a}_{K}$ positively span $\mathbb{R}^{N}$ if for every $\boldsymbol{a} \in \mathbb{R}^{N} \backslash\{\boldsymbol{0}\}$ there exists $k \in\{1, \ldots, K\}$ such that $\boldsymbol{a}^{\top} \boldsymbol{a}_{k}>0$. (2) If nonzero vectors $\boldsymbol{a}_{1}, \ldots, \boldsymbol{a}_{K}$ already span $\mathbb{R}^{N}$, then they positively span $\mathbb{R}^{N}$ if $\mathbf{0}$ admits a nontrivial positive representation, that is, if there exist nonnegative $\lambda_{1}, \ldots, \lambda_{K}$, not all 0 , such that $\lambda_{1} a_{1}+\cdots+\lambda_{K} a_{K}=0$.

DEFINITION 2.12 [The a priori boundedness (AB) condition]. We say that $\boldsymbol{f}$ satisfies the condition $(A B)$ if there exist a deterministic function $l \in \mathbb{L}^{1}[0, T]$, and a set $\boldsymbol{a}_{1}, \ldots, \boldsymbol{a}_{K}$, which positively spans $\mathbb{R}^{N}$, such that

$$
\boldsymbol{a}_{k}^{\top} \boldsymbol{f}(t, x, \boldsymbol{y}, \boldsymbol{z}) \leq l(t)+\frac{1}{2}\left|\boldsymbol{a}_{k}^{\top} \boldsymbol{z}\right|^{2} \quad \text { for all }(t, x, \boldsymbol{y}, \boldsymbol{z}) \text { and } k=1, \ldots, K \text {. }
$$

We say that $\boldsymbol{f}$ satisfies the weak condition $(A B)$-abbreviated as $(w A B)$ - if there exist Borel functions $\boldsymbol{L}_{k}:[0, T] \times \mathbb{R}^{d} \times \mathbb{R}^{N \times d} \rightarrow \mathbb{R}^{d}$, for $k=1, \ldots, K$, such that $\left|\boldsymbol{L}_{k}(t, x, z)\right| \leq C(1+|z|)$ for some constant $C$ and

$$
\begin{gathered}
\boldsymbol{a}_{k}^{\top} \boldsymbol{f}(t, x, \boldsymbol{y}, \boldsymbol{z}) \leq l(t)+\frac{1}{2}\left|\boldsymbol{a}_{k}^{\top} \boldsymbol{z}\right|^{2}+\boldsymbol{a}_{k}^{\top} \boldsymbol{z} \boldsymbol{L}_{k}(t, x, \boldsymbol{z}) \\
\text { for all }(t, x, \boldsymbol{y}, z) \text { and } k=1, \ldots, K
\end{gathered}
$$

REMARK 2.13. The constant $\frac{1}{2}$ in (2.9) is simply a convenient choice for later use; it can easily be replaced by any other constant by scaling. Furthermore, conditions $(\mathrm{AB})$ and $(\mathrm{WAB})$ are invariant under invertible linear transformation of $\mathbb{R}^{N}$. More precisely, suppose that $f$ satisfies (wAB) with $l \in \mathbb{L}^{1}[0, T]$, the positively-spanning set $\boldsymbol{a}_{1}, \ldots, \boldsymbol{a}_{K}$ and the functions $\left\{\boldsymbol{L}_{k}\right\}$, and that $\Sigma: \mathbb{R}^{N} \rightarrow \mathbb{R}^{N}$ 
is an invertible linear map. Then the generator of the transformed system, namely $\tilde{\boldsymbol{f}}(t, x, \tilde{\boldsymbol{y}}, \tilde{\boldsymbol{z}}):=\Sigma \boldsymbol{f}(t, x, \boldsymbol{y}, \boldsymbol{z})$, satisfies (wAB) with the same $l, \tilde{\boldsymbol{L}}_{k}(t, x, \tilde{\boldsymbol{y}}, \tilde{\boldsymbol{z}})=$ $\boldsymbol{L}_{k}(t, x, \boldsymbol{y}, \boldsymbol{z})$, and transformed (but still positively spanning) set $\left(\Sigma^{-1}\right)^{\top} \boldsymbol{a}_{k}$, $k=1, \ldots, K$.

THEOREM 2.14. [Existence under $(\mathrm{BF})+(\mathrm{AB})$ ] Suppose that $\boldsymbol{f}$ satisfies conditions $(B F)$ and $(A B)$, and that $g \in C_{\mathrm{loc}, b_{0}}^{\left\{\alpha_{n}\right\}}$ for some $b_{0}$ and it satisfies $\lim _{|x| \rightarrow \infty}|\boldsymbol{g}(x)| /|x|^{2}=0$. Then the system (2.2) admits a locally Hölderian solution $(\boldsymbol{v}, \boldsymbol{w})$, that is, $\boldsymbol{v} \in C_{\mathrm{loc}, b_{0}}^{\left\{\alpha_{n}\right\}}$ for some sequence $\left\{\alpha_{n}^{\prime}\right\}$ in $(0,1]$. When $\boldsymbol{g}$ is bounded, the condition $(A B)$ can be replaced by $(w A B)$ and $(\boldsymbol{v}, \boldsymbol{w})$ is a bounded bmo-solution.

[Uniqueness under $(\mathrm{BF})+(\mathrm{wAB})]$ Suppose that:

1. $\boldsymbol{g} \in C_{\mathrm{loc}}^{\left\{\alpha_{n}\right\}} \cap \mathbb{L}^{\infty}$ for some sequence $\left\{\alpha_{n}\right\} \in(0,1]$;

2. (wAB) is satisfied, and $(B F)$ is satisfied with the constants $\left\{C_{n}\right\}$ and functions $\left\{\kappa_{n}\right\}$ independent of $n$;

3. $\boldsymbol{f}$ does not depend on $\boldsymbol{y}, \boldsymbol{f}(\cdot, \cdot, 0)$ is bounded, and there exists a constant $M$ such that $\left|\boldsymbol{f}(t, x, \boldsymbol{z})-\boldsymbol{f}\left(t, x, \boldsymbol{z}^{\prime}\right)\right| \leq M\left(|\boldsymbol{z}|+\left|\boldsymbol{z}^{\prime}\right|\right)\left|\boldsymbol{z}-\boldsymbol{z}^{\prime}\right|$ for all $t \in[0, T], x \in \mathbb{R}^{d}$, $\boldsymbol{z}, \boldsymbol{z}^{\prime} \in \mathbb{R}^{N \times d}$.

Then the solution $(\boldsymbol{v}, \boldsymbol{w})$ is unique in the class of bounded continuous solutions.

REMARK 2.15. Here are two extensions of Theorem 2.14 which, for the sake of simplicity of presentation, we did not put into its statement. They will be proved, however, along with Theorem 2.14, below:

1. When $\boldsymbol{g}$ is bounded, the conclusions of Theorem 2.14 hold if the equality in (2.8) holds only approximately, namely if, for each $n \in \mathbb{N}$, there exists a sufficiently small $\varepsilon_{n}$ such that

$$
\begin{aligned}
& |\boldsymbol{f}(t, x, \boldsymbol{y}, \boldsymbol{z})-\operatorname{diag}(z \mathbf{l}(t, x, \boldsymbol{y}, \boldsymbol{z}))-\mathbf{q}(t, x, \boldsymbol{y}, \boldsymbol{z})-\mathbf{s}(t, x, \boldsymbol{y}, \boldsymbol{z})-\mathbf{k}(t, x)| \\
& \quad \leq \varepsilon_{n}|z|^{2}
\end{aligned}
$$

holds on $[0, T] \times B_{n}\left(b_{0}\right) \times \mathbb{R}^{N} \times \mathbb{R}^{N \times d}$. How small this $\varepsilon_{n}$ needs to be depends on the constants $\left\{C_{n}\right\}$ in the condition (BF), on $\|\boldsymbol{v}\|_{\mathbb{L}^{\infty}}$ [which, in turn, depends on $\|\boldsymbol{g}\|_{\mathbb{L}^{\infty}}$ and the functions and constants appearing in condition (wAB)], as well as the universal constants $(\Lambda, T, d, N$, etc.). In general, it is possible to obtain an explicit expression for an estimate of $\varepsilon_{n}$ by keeping track of the explicit values of the constants involved in the proof, but we do not pursue that here. The case in which such an explicit expression may prove to be useful is when $\mathbf{I}=\mathbf{0}, \mathbf{q}=\mathbf{s}=\mathbf{0}$ and $\mathbf{k}=\mathbf{0}$, that is, when $\boldsymbol{f}$ is of general structure, but satisfies a smallness assumption. This case allows for an especially simple treatment; indeed, to construct a global Lyapunov pair, it suffices to pick

$$
h(\boldsymbol{y})=\frac{1}{2}|\boldsymbol{y}|^{2}, \quad k \equiv 0 \quad \text { so that } D^{2} h=I_{d} \text { and } D h(\boldsymbol{y})=\boldsymbol{y} .
$$


Then

$$
\frac{1}{2} D^{2} h:\langle z, z\rangle_{a}=\frac{1}{2} \sum_{i=1}^{D}\left|z^{i} \sigma\right|^{2} \geq \frac{1}{2} \Lambda^{-1} \sum_{i=1}^{D}\left|z^{i}\right|^{2} \quad \text { but } D h \boldsymbol{f} \leq \varepsilon|\boldsymbol{y}||\boldsymbol{z}|^{2} .
$$

Therefore, it suffices to require $\varepsilon<\left(4 \Lambda\|\boldsymbol{v}\|_{\mathbb{L}^{\infty}}\right)^{-1}$ so that $(h, 0) \in \mathbf{L y}\left(\boldsymbol{f}, 2\|\boldsymbol{v}\|_{\mathbb{L}^{\infty}}\right)$. This recovers the situation in [53] where solutions to parabolic systems of PDEs were constructed under a parallel "smallness" condition.

2. Suppose that some component of $g\left(X_{T}\right)$, say the $j$ th, has a bounded Malliavin derivative and $f^{j}$ does not depend on $z^{i}$ for $i \neq j$. It is known then (see [11] and [17] for sufficient conditions on $X, f^{j}$ and $g^{j}$ ) that the $j$ th component $\boldsymbol{Z}^{j}$ of the solution is bounded, too. In this case, Theorem 2.14 still holds if any locally bounded function of $z^{j}$ is added to the right-hand side of (2.9) and (2.10).

3. Examples. We illustrate the strength of our results by considering four different classes of BSDE systems arising from game theory, geometry, mathematical economics and mathematical finance. Proofs of all statements are postponed until Section 5.

3.1. Incomplete stochastic equilibria. The existence and properties of equilibrium (market-clearing) asset-price dynamics in financial markets is one of the central problems in financial economics and mathematical finance. While the socalled complete market case has been fully understood, the incomplete market case has been open since early 1990s. A stochastic equilibrium among $N$ heterogeneous agents in incomplete markets has been considered in [40]. There the filtration is generated by a 2-dimensional Brownian motion $W=\left(B, B^{\perp}\right)$, where the first component drives the price of a tradable asset but both components can determine the size of agents' random endowment. Preference of agents are modeled by exponential utilities with heterogeneous risk-tolerance coefficients. An equilibrium is a pair consisting of an asset-price process and agents' trading strategies such that every agent maximizes the expected utility from trading and random endowment, meanwhile supply equals to demand (market clears); cf. [40], Definition 1.1 .

In this setting, [40] considered the following system of quadratic BSDE:

$$
d \boldsymbol{Y}_{t}=\boldsymbol{\mu}_{t} d B_{t}+\boldsymbol{v}_{t} d B_{t}^{\perp}+\left(\frac{1}{2} \boldsymbol{v}_{t}^{2}-\frac{1}{2} \boldsymbol{A}\left[\boldsymbol{\mu}_{t}\right]^{2}+\boldsymbol{A}\left[\boldsymbol{\mu}_{t}\right] \boldsymbol{\mu}_{t}\right) d t, \quad \boldsymbol{Y}_{T}=\boldsymbol{G}
$$

where $\boldsymbol{A}[\boldsymbol{\mu}]=\sum_{i=1}^{N} \alpha^{i} \mu^{i}$ for a sequence of constants $\left(\alpha^{i}\right)$ with $\alpha^{i} \in(0,1)$ and $\sum_{i=1}^{N} \alpha^{i}=1$. It is proved in [40], Theorem 1.6, that equilibria one-to-one correspond to solutions of (3.1) with $(\boldsymbol{\mu}, \boldsymbol{v}) \in$ bmo. Moreover, in an equilibrium, each component of $\boldsymbol{Y}$ represents the certainty equivalence of each agent. However, when it comes to the existence and uniqueness of solutions, certain "smallness-type" of 
conditions need to be assumed; either $\|\boldsymbol{G}\|_{\mathbb{L}^{\infty}}$ is sufficiently small or $T$ is sufficiently small; cf. [40], Corollaries 2.6 and 2.7. In the Markovian setting, existence of solutions was also established for sufficiently small $T$ in [60] in a similar model, and [59] and [20], Theorem 3.1.

The following result establishes global existence and uniqueness of equilibrium in a Markovian setting with bounded random endowment. Here, $X$ is the solution of (2.1) with $W=\left(B, B^{\perp}\right), b:[0, T] \times \mathbb{R}^{d} \rightarrow \mathbb{R}^{d}$, and $\sigma:[0, T] \times \mathbb{R}^{d} \rightarrow \mathbb{R}^{d \times 2}$ satisfying conditions (1)-(3) after (2.1).

THEOREM 3.1 [Existence and uniqueness of incomplete stochastic equilibria]. Suppose that the terminal condition is of the form $\boldsymbol{G}=\boldsymbol{g}\left(X_{T}\right)$ for some $g \in C_{\mathrm{loc}}^{\left\{\alpha_{n}\right\}} \cap \mathbb{L}^{\infty}$. Then the system (3.1) admits a unique bounded continuous solution. Consequently, an incomplete stochastic equilibrium in the setting of [40] exists and is unique in the class of equilibria in which each agent's certaintyequivalence process is a continuous function of time and the state $X$.

REMARK 3.2. When $\boldsymbol{g}$ is of merely subquadratic growth, the system (3.1) still admits a locally Hölderian solution, but the martingale part associated to this solution may not have enough integrability to be identified with an equilibrium.

3.2. Martingales on manifolds. It is well known that semimartingales can be defined on arbitrary differentiable manifolds, but that martingales require additional structure, namely that of a connection (if one wants a Brownian motion, one needs a full Riemannian metric). We refer the reader to the books [28] and [35] for more details.

In the flat (Euclidean) case, martingales are easily constructed from their terminal values by a simple process of filtering, that is, computing conditional expectation. When the underlying filtration is Brownian, one can, additionally, build this martingale from the given Brownian motion via the martingale representation theorem; this amounts to a solution to a linear system of BSDE.

If the geometry is not flat, one cannot simply filter anymore, but as it turns out, the problem can still be formulated in terms of a system of BSDE. This system, however, is no longer linear and the existence of its solution has been a subject of extensive study (see, e.g., $[9,21]$ and [10]).

Before we write down this system, we set the stage by assuming that a $d$ dimensional Brownian motion $W$ is given, and that the target space is an $N$ dimensional differentiable manifold $M$, without boundary, endowed with an affine connection. This connection, $\Gamma$, is described in coordinates by its Christoffel symbols $\Gamma_{i j}^{k}$; we assume these are all Lipschitz on compact sets, but not necessarily differentiable (as we will not be needing the concept of curvature). 
The martingale property on a manifold with a connection $\Gamma$ can be formulated in many ways - we prefer to give the one that resembles a characterization in the flat case; we say that a continuous $M$-valued semimartingale $\boldsymbol{Y}$ is a $\Gamma$-martingale (with respect to the natural filtration of $W$ ) if

$$
f\left(\boldsymbol{Y}_{t}\right)-\frac{1}{2} \int_{0}^{t} \operatorname{Hess} f\left(d \boldsymbol{Y}_{t}, d \boldsymbol{Y}_{t}\right), \quad t \in[0, T]
$$

is a local martingale for each smooth real-valued $f$. Here, Hess $f$ is the (covariant) Hessian of $f$, that is, a $(0,2)$-tensor, given in our coordinate chart by

$$
(\text { Hess } f)_{i j}(\boldsymbol{y})=D_{i j} f(\boldsymbol{y})-\Gamma_{i j}^{k}(\boldsymbol{y}) D_{k} f(\boldsymbol{y}) \text {. }
$$

We refer the reader to [28], page 23, for the definition of quadratic variation with respect to a $(0,2)$-tensor field (such as Hess) on a manifold. Itô's formula immediately implies that $\boldsymbol{Y}$ is a $\Gamma$-martingale if its coordinate representation admits the following semimartingale decomposition:

$$
d Y_{t}^{k}=-\frac{1}{2} \sum_{i, j=1}^{d} \Gamma_{i j}^{k}\left(\boldsymbol{Y}_{t}\right)\left(\boldsymbol{Z}_{t}^{i}\right)^{\top} \boldsymbol{Z}_{t}^{j} d t+\boldsymbol{Z}_{t}^{k} d W_{t}, \quad k=1, \ldots, N,
$$

where, as usual, $\boldsymbol{Z}^{j}$ denotes the $j$ th row of the $N \times d$-matrix-valued process $\boldsymbol{Z}$.

For simplicity, and without too great a loss of generality, we assume that the given terminal value $\boldsymbol{G}$ of the martingale we want to construct is of the form $\boldsymbol{g}\left(W_{T}\right)$. Furthermore, we assume that the image of $\boldsymbol{g}$ is localized in the following way: there exists a convex and compact set $M_{0}$, covered by the image $V \subseteq M$ of a single chart, with coordinates $\boldsymbol{y}=\left(y_{1}, \ldots, y_{N}\right)$, such that $\boldsymbol{g}(x) \in M_{0}$, for all $x \in R^{d}$. This way, we can work in a single coordinate chart, as if $M$ itself were an open set of $\mathbb{R}^{N}$ and, in fact, assume that $M=\mathbb{R}^{N}$. Also since we only care about the connection in a neighborhood of $M_{0}$, we assume that the Christoffel symbols are globally Lipschitz.

As in [21], we make the following assumption on the geometry of $M$ around the image of $g$.

Assumption 3.3 (Double convexity). There exists a convex function $\phi \in$ $C^{2}\left(\mathbb{R}^{N}\right)$ such that:

1. $M_{0}=\phi^{-1}((-\infty, 0])$, and

2. Hess $\phi$ is nonnegative definite ( $\phi$ is geodesically convex), and strictly positive definite on some neighborhood of $M_{0}$.

Applying Theorem 2.8 to the current setting, we obtain the following result.

Proposition 3.4. If $\boldsymbol{g} \in C_{\mathrm{loc}}^{\left\{\alpha_{n}\right\}}\left(\mathbb{R}^{d}\right)$ and Assumption 3.3 holds, there exists a $\Gamma$-martingale $\left\{\boldsymbol{Y}_{t}\right\}_{t \in[0, T]}$ with $\boldsymbol{Y}_{T}=\boldsymbol{g}\left(W_{T}\right)$ which takes values in $M_{0}$, for all $t \in[0, T]$. 
REMARK 3.5 .

1. While the detailed proof of Proposition 3.4 above is postponed until Section 5, we comment briefly on the interpretation of Lyapunov pairs in this special case. What makes it especially convenient is the fact that the driver $\boldsymbol{f}$ depends on $\boldsymbol{Z}$ only through the symmetric matrix $\boldsymbol{Z}^{\top} \boldsymbol{Z}$. A simple computation shows that $(h, 0)$ is a $c$-Lyapunov pair if (and only if) the matrix

$$
\left(D_{i j}^{2} h(\boldsymbol{y})-\sum_{k} D_{k} h(\boldsymbol{y}) \Gamma_{i j}^{k}(\boldsymbol{y})\right)_{i j}, \quad i, j=1, \ldots, d,
$$

is strictly positive definite for all $|\boldsymbol{y}| \leq c$. Equivalently, Hess $h(\boldsymbol{y}) \succeq 0$, that is, $h$ is (geodesically) strictly convex (see, e.g., Chapter 3 of [57] for a detailed discussion of convexity on Riemannian manifolds). This characterization fits perfectly with our interpretation of Lyapunov functions as "submartingale" functions.

2. Unlike in the flat case, where convex functions abound, the very existence of (geodesically) convex functions depends on geometric properties on $M$. We do not go into details, but note that smooth nontrivial global convex functions always exist on complete, simple-connected Riemannian manifolds of nonpositive sectional curvature (Cartan-Hadamard manifolds); cf. [41]. In the general case, one can always find a convex function locally, but it is not hard to see that compact Riemannian manifolds, for example, never admit nonconstant global convex functions. We refer the reader to [57] for a thorough treatment of geodesic convexity.

3. The condition of double convexity has been imposed in [21] to construct not-necessarily-Markovian martingales with values in manifolds with connections. Our construction not only recovers some of the results from [21] in the Markovian case, but also gives a partial positive answer to Conjecture 7.2, page 1257. Indeed, Proposition 3.4 does not require $M_{0}$ to have doubly convex geometry, that is, does not assume Conditions 5.1 and 6.1 in [21], Theorem 7.1.

4. Without too much work, Proposition 3.4 can be extended in several directions. First, the flat Brownian motion $W$ can be replaced by a Brownian motion on a Riemannian manifold (with metric $g$ ); indeed, one simply needs to solve a modified version of BSDE 3.2 driven by a driftless diffusion whose dispersion coefficient $\sigma$ relates to the underlying metric as $\sigma \sigma^{\top}=g^{-1}$. Moreover, under appropriate growth conditions, the linear connection $\Gamma$ can be replaced by a nonlinear one, of the form $\Gamma=\Gamma\left(\boldsymbol{y}, \boldsymbol{Z}^{\top} \boldsymbol{Z}\right)$.

5. Seen as a map between manifolds, a function which transforms a Browninan motion into a martingale is called harmonic. Our BSDE (3.2) corresponds to the parabolic system introduced by [25] in order to show that, under certain geometric conditions, harmonic maps exist within each homotopy class (see [2], Chapter 10, for a detailed treatment of this fascinating problem). 
3.3. A stochastic game with cooperation or hindrance. Our next example concerns a finite-horizon stochastic differential game, which is inspired by a boundeddomain discounted game treated in [6]. For simplicity of presentation, we assume there are only 2 players whom we call Player 1 and Player 2 . On a $d$-dimensional Brownian filtration, these players choose two $\mathbb{R}^{d}$-valued processes, namely $\boldsymbol{\mu}$ and $\boldsymbol{v}$ in bmo, as their respective controls. These affect the state $X$ through its drift in the following way:

$$
d X_{t}^{(\mu, v)}=\left(b\left(X_{t}^{(\mu, v)}\right)+\mu_{t}+v_{t}\right) d t+d W_{t}^{(\mu, v)}, \quad X_{0}^{(\mu, v)}=x,
$$

where $b: \mathbb{R}^{d} \rightarrow \mathbb{R}^{d}$ is a bounded Lipschitz vector field, and $W^{(\mu, \nu)}=W-$ $\int_{0}^{\cdot}\left(\boldsymbol{\mu}_{u}+\boldsymbol{v}_{u}\right) d u$ is a Brownian motion under the probability measure $\mathbb{P}^{(\mu, v)}$ defined via $d \mathbb{P}^{(\mu, \nu)} / d \mathbb{P}=\left(\int\left(\boldsymbol{\mu}_{u}+\boldsymbol{v}_{u}\right)^{\top} d W_{u}\right)_{T}$. Given a constant $\theta$ - which we term the cooperation penalty - and integrable-enough functions $h^{i}, g^{i}: \mathbb{R}^{d} \rightarrow \mathbb{R}, i=1,2$, the cost of player $i$ with the initial state $x$ at time $t=0$ is defined as

$$
\begin{aligned}
& J^{i}(0, x, \mu, v) \\
& \quad=\mathbb{E}^{(x, \mu, \nu)}\left[\int_{0}^{T}\left(h^{i}\left(X_{u}\right)+\frac{1}{2}\left|\boldsymbol{\mu}_{u}\right|^{2}+\theta \boldsymbol{\mu}_{u}^{\top} \boldsymbol{v}_{u}\right) d t+g^{i}\left(X_{T}\right)\right], \quad i=1,2,
\end{aligned}
$$

where the expectation is taken with respect to $\mathbb{P}^{(x, \mu, v)}$. It is clear from its form how large positive values of the parameter $\theta$ incentivize the players to push in opposing directions, while the large negative values motivate them to cooperate. A Nash equilibrium between these two players is a pair $(\hat{\boldsymbol{\mu}}, \hat{\boldsymbol{v}})$ of controls with the property that, for any $\boldsymbol{\mu}, \boldsymbol{v} \in$ bmo, we have

$$
J^{1}(0, x, \hat{\boldsymbol{\mu}}, \hat{\boldsymbol{v}}) \leq J^{1}(0, x, \boldsymbol{\mu}, \hat{\boldsymbol{v}}) \quad \text { and } \quad J^{2}(0, x, \hat{\boldsymbol{\mu}}, \hat{\boldsymbol{v}}) \leq J^{2}(0, x, \hat{\boldsymbol{\mu}}, \boldsymbol{v}),
$$

and $\left(J^{1}, J^{2}\right)(\cdot, \cdot, \hat{\boldsymbol{\mu}}, \hat{\boldsymbol{v}})$ is called the value of this equilibrium.

We recast the problem as a BSDE system by introducing the Lagrangians of the two players

$$
\begin{aligned}
& L^{1}(\boldsymbol{\mu}, \boldsymbol{v}, \boldsymbol{p})=\frac{1}{2}|\boldsymbol{\mu}|^{2}+\theta \boldsymbol{\mu} \cdot \boldsymbol{v}+\boldsymbol{p}^{1}(\boldsymbol{\mu}+\boldsymbol{v}) \quad \text { and } \\
& L^{2}(\boldsymbol{\mu}, \boldsymbol{v}, \boldsymbol{p})=\frac{1}{2}|\boldsymbol{v}|^{2}+\theta \boldsymbol{\mu} \cdot \boldsymbol{v}+\boldsymbol{p}^{2}(\boldsymbol{\mu}+\boldsymbol{v}),
\end{aligned}
$$

where $\boldsymbol{p}^{i}$ is $i$ th row vector of $\boldsymbol{p}$. When $\theta \neq \pm 1$, the minimizers are given by

$$
\begin{aligned}
& \hat{\boldsymbol{\mu}}(\boldsymbol{p})=\frac{\theta}{(1+\theta)(1-\theta)}\left(\boldsymbol{p}^{1}+\boldsymbol{p}^{2}\right)-\frac{1}{1-\theta} \boldsymbol{p}^{1} \text { and } \\
& \hat{\boldsymbol{v}}(\boldsymbol{p})=\frac{\theta}{(1+\theta)(1-\theta)}\left(\boldsymbol{p}^{1}+\boldsymbol{p}^{2}\right)-\frac{1}{1-\theta} \boldsymbol{p}^{2} .
\end{aligned}
$$


Setting $L^{i}(\boldsymbol{p})=L^{i}(\hat{\boldsymbol{\mu}}(\boldsymbol{p}), \hat{\boldsymbol{v}}(\boldsymbol{p}), \boldsymbol{p})$ and $\boldsymbol{L}=\left(L^{1}, L^{2}\right)^{\top}$, we pose the following BSDE:

$$
\begin{aligned}
d \boldsymbol{Y}_{t} & =-\boldsymbol{f}\left(X_{t}, \boldsymbol{Z}_{t}\right) d t+\boldsymbol{Z}_{t} d W_{t}, \\
\boldsymbol{Y}_{T} & =\boldsymbol{g}\left(X_{T}\right) \quad \text { where } \boldsymbol{f}(x, z)=\boldsymbol{h}(x)+\boldsymbol{L}(z),
\end{aligned}
$$

with the state process given by $d X_{t}=b\left(X_{t}\right) d t+d W_{t}$ on the (augmented) filtration generated by $W$. The following result establishes a unique bounded Hölderian solution, which corresponds to a Nash equilibrium.

Proposition 3.6. Assume that $\boldsymbol{h} \in \mathbb{L}^{\infty}$ and $\boldsymbol{g} \in C_{\mathrm{loc}}^{\left\{\alpha_{n}\right\}} \cap \mathbb{L}^{\infty}$. When $-1 \neq$ $\theta \leq 1 / 2$ or $\theta>1$, equation (3.5) admits a unique bounded continuous solution $(\boldsymbol{v}, \boldsymbol{w})$. Moreover, the pair $(\hat{\boldsymbol{\mu}}(\boldsymbol{Z}), \hat{\boldsymbol{v}}(\boldsymbol{Z}))$, where $\boldsymbol{Z}=\boldsymbol{w}(\cdot, X)$, is in bmo and enacts a Nash equilibrium with the value $\boldsymbol{v}$.

3.4. Risk-sensitive nonzero-sum stochastic games. Next, we consider a risksensitive stochastic game between 2 players studied in [26]. Let $U$ and $V$ be two compact metric spaces. Player 1 (resp., player 2) chooses a $U$-valued (resp., $V$ valued) control process $\boldsymbol{\mu}$ (resp. $\boldsymbol{v}$ ), which affects the state $X$ in the following way:

$$
d X_{t}^{(\mu, v)}=b\left(t, X_{t}^{(\mu, v)}, \boldsymbol{\mu}_{t}, \boldsymbol{v}_{t}\right) d t+\sigma\left(t, X_{t}^{\mu, \nu}\right) d W_{t}^{(\mu, v)},
$$

where $b:[0, T] \times \mathbb{R}^{d} \times U \times V \rightarrow \mathbb{R}^{d}$ is a bounded measurable vector field, $\sigma$ satisfies conditions (2) and (3) after (2.1), and $X$ is understood as the unique weak solution of the previous stochastic differential equation. Given measurable functions $h^{i}:[0, T] \times \mathbb{R}^{d} \times U \times V \rightarrow \mathbb{R}$ and $g^{i}: \mathbb{R}^{d} \rightarrow \mathbb{R}$ with enough integrability, the cost of player $i$ with the initial state $x$ at time $t=0$ is defined as

$$
J^{i}(0, x, \boldsymbol{\mu}, \boldsymbol{v})=\mathbb{E}^{(x, \mu, v)}\left[\exp \left(\int_{0}^{T} h^{i}\left(u, X_{u}, \boldsymbol{\mu}_{u}, \boldsymbol{v}_{u}\right) d u+g^{i}\left(X_{T}\right)\right)\right], \quad i=1,2 .
$$

The problem is to find a Nash equilibrium $(\hat{\boldsymbol{\mu}}, \hat{\boldsymbol{v}})$ satisfying (3.3). To solve it, we define the Hamiltonian function

$$
\begin{aligned}
& H^{1}(t, x, \boldsymbol{\mu}, \boldsymbol{v})=\boldsymbol{\mu} \sigma^{-1}(t, x) b(t, x, \boldsymbol{\mu}, \boldsymbol{v})+h^{1}(t, x, \boldsymbol{\mu}, \boldsymbol{v}) \quad \text { and } \\
& H^{2}(t, x, \boldsymbol{\mu}, \boldsymbol{v})=\boldsymbol{v} \sigma^{-1}(t, x) b(t, x, \boldsymbol{\mu}, \boldsymbol{v})+h^{2}(t, x, \boldsymbol{\mu}, \boldsymbol{v}),
\end{aligned}
$$

and assume the generalized Issac's condition holds, that is, there exists two measurable functions $\hat{\boldsymbol{\mu}}(t, x, z)$ and $\hat{\boldsymbol{v}}(t, x, z)$ such that

$$
\begin{aligned}
& H^{1}\left(t, x, z^{1}, \hat{\boldsymbol{\mu}}(t, x, z), \hat{\boldsymbol{v}}(t, x, z)\right) \leq H^{1}\left(t, x, z^{1}, \boldsymbol{\mu}, \hat{\boldsymbol{v}}(t, x, z)\right) \quad \text { and } \\
& H^{2}\left(t, x, z^{2}, \hat{\boldsymbol{\mu}}(t, x, z), \hat{\boldsymbol{v}}(t, x, z)\right) \leq H^{2}\left(t, x, z^{2}, \hat{\boldsymbol{\mu}}(t, x, z), \boldsymbol{v}\right),
\end{aligned}
$$

for any $(t, x, \boldsymbol{z}, \boldsymbol{\mu}, \boldsymbol{v}) \in[0, T] \times \mathbb{R}^{d} \times \mathbb{R}^{2 \times d} \times U \times V$. Denote $\hat{H}^{1}(t, x, z)=$ $H^{1}\left(t, x, z^{1}, \hat{\boldsymbol{\mu}}(t, x, z), \hat{\boldsymbol{v}}(t, x, \boldsymbol{z})\right) \quad$ and $\quad \hat{H}^{2}(t, x, z)=H^{2}\left(t, x, z^{2}, \hat{\boldsymbol{\mu}}(t, x, z)\right.$, 
$\hat{\boldsymbol{v}}(t, x, z))$. We consider the following system of BSDE:

$$
\begin{aligned}
d Y_{t}^{i} & =-\left(\hat{H}^{i}\left(t, X_{t}, \boldsymbol{Z}_{t}\right)+\frac{1}{2}\left|\boldsymbol{Z}_{t}^{i}\right|^{2}\right) d t+Z_{t}^{i} d W_{t}, \\
Y_{T}^{i} & =g^{i}\left(X_{T}\right), \quad i=1,2 .
\end{aligned}
$$

Proposition 3.7. Assume that $g^{i} \in C_{\mathrm{loc}}^{\left\{\alpha_{n}\right\}} \cap \mathbb{L}^{\infty}, h^{i} \in \mathbb{L}^{\infty}$ and $\hat{H}^{i}$ is continuous, for $i=1,2$. Moreover, $b$ has at most linear growth in $(\boldsymbol{\mu}, \boldsymbol{v})$, and $(\hat{\boldsymbol{\mu}}, \hat{\boldsymbol{v}})$ has at most linear growth in $z$, both uniformly in $(t, x)$. Then (3.6) admits a unique bounded continuous solution $(\boldsymbol{v}, \boldsymbol{w})$. Moreover, $(\hat{\boldsymbol{\mu}}(\cdot, \cdot, \boldsymbol{w}), \hat{\boldsymbol{v}}(\cdot, \cdot, \boldsymbol{w}))$ is a Nash equilibrium with value $\left(\exp \left(v^{1}\right), \exp \left(v^{2}\right)\right)$.

REMARK 3.8. A solution to (3.6) was constructed in [26], Theorem 5.3, in the case of a bounded $b$ and a bounded (but not necessarily continuous) terminal condition $\boldsymbol{g}$. When $\boldsymbol{g}$ is locally Hölder, our result shows that the solution is also locally Hölder [cf. Remark 2.6 part (2)]. Moreover, when $\boldsymbol{g}$ is of merely subquadratic and $b$ is bounded, our result still ensures the existence of locally Hölderian solution to (3.6).

The system (3.6) belongs to the diagonally quadratic class studied recently in [37], whose Theorem 2.7 implies the existence of a unique bounded solution of (3.6) with the non-Markovian bounded terminal condition.

3.5. A scalar example with unbounded coefficients. Given continuous functions $f, g: \mathbb{R}^{d} \rightarrow \mathbb{R}$, with $g \in C_{\text {loc }}^{\left\{\alpha_{n}\right\}} \cap \mathbb{L}^{\infty}$, but $f$ possibly unbounded, we consider the BSDE

$$
d Y_{t}=-\frac{1}{2} f\left(X_{t}\right)\left|Z_{t}\right|^{2} d t+Z_{t} d W_{t}, \quad Y_{T}=g\left(X_{T}\right) .
$$

An equation of this type played a central role in a recent solution [14] of a longstanding open problem of [54]. Since the "coefficient" $f$ in front of the quadratic nonlinearity is unbounded, the generator of (3.7) does not satisfy the standard quadratic growth bound in $z$ (as presented, e.g., in [42]).

Our Theorem 2.8 implies that (3.7) admits a bounded locally Hölderian solution. Indeed, consider a sequence $\left\{f^{m}\right\}$ of bounded Lipschitz approximations of $f$ such that $\lim _{m \rightarrow \infty} f^{m}(x)=f(x)$ for any $x \in \mathbb{R}^{d}$, and the approximating BSDE

$$
\begin{aligned}
d Y_{t}^{m} & =-\frac{1}{2} f^{m}\left(X_{t}\right)\left(\left|Z_{t}^{m}\right|^{2} \wedge m\right) d t+Z_{t}^{m} d W_{t}, \\
Y_{T}^{m} & =g\left(X_{T}\right), \quad m \in \mathbb{N} .
\end{aligned}
$$

Standard Lipschitz theory implies that (3.8) admits a unique bounded continuous solution $\left(v^{m}, w^{m}\right)$. Moreover, the generator of (3.8) satisfies the condition (BF) of Definition 2.10 with $\mathbf{I}^{m}=\mathbf{s}^{m}=\mathbf{k}^{m}=0$; the component $\mathbf{q}^{m}$ satisfies the quadratictriangular growth condition on each $B_{n}$ uniformly in $m$. By Proposition 2.11 above, 
$f$ admits a local $\left\{c_{n}\right\}$-Lyapunov sequence, for each $\left\{c_{n}\right\}$. To establish a priori boundedness, we rewrite (3.8) as

$$
d Y_{t}^{m}=Z_{t}^{m}\left[-\frac{1}{2} f^{m}\left(X_{t}\right) \frac{\left|Z_{t}^{m}\right|^{2} \wedge m}{\left|Z^{m}\right|^{2}}\left(Z_{t}^{m}\right)^{\top} d t+d W_{t}\right], \quad Y_{T}^{m}=g\left(X_{T}\right) .
$$

Since $f^{m}$ and $g$ are bounded, a simple measure-change argument implies that $\left\|v^{m}\right\|_{\mathbb{L}^{\infty}} \leq\|g\|_{\mathbb{L}^{\infty}}$. Therefore, it is enough to pick a local $\left(\|g\|_{\mathbb{L}^{\infty}}\right)$-Lyapunov pair to establish the existence of a bounded locally Hölderian solution by Theorem 2.8. It is worth noting that our uniqueness results do not apply in this case. In fact, as far as we know, no general-purpose uniqueness result is known for the BSDE of this type.

REMARK 3.9. The techniques of the present paper, geared toward systems of equations, have limited impact in the one-dimensional case where powerful methods based on comparison principle apply. To illustrate that point, we note that the existence of a Markovian solution for (3.7) can also be established using a localization technique of [12] or from a forward point of view, as in [5], as follows. With $f^{m, p}(x)=(-p) \vee f(x) \wedge m$ for $m, p \in \mathbb{N}$, the approximating BSDE

$$
d Y_{t}^{m, p}=-\frac{1}{2} f^{m, p}\left(X_{t}\right)\left|Z_{t}^{m, p}\right|^{2} d t+Z_{t}^{m, p} d W_{t}, \quad Y_{T}^{m, p}=g\left(X_{T}\right),
$$

admits a unique bounded continuous solution $\left(v^{m, p}, w^{m, p}\right)$; $\mathrm{cf}$. [42], Theorems 2.3 and 3.7. Define the exit time $\tau_{n}=\inf \left\{u \geq 0: X_{u} \notin B_{n}\right\}$. Comparison theorem for quadratic BSDE implies $v^{m, p+1} \leq v^{m, p} \leq v^{m+1, p}$. It then follows from the monotone stability of quadratic BSDE (cf. [42], Proposition 2.4) that $Y_{\cdot \wedge \tau_{n}}^{m, p}=$ $v^{m, p}\left(\cdot, X_{\cdot}^{\tau_{n}}\right)$ increasingly converges to some process $Y_{\cdot \wedge \tau_{n}}^{p}$ as $m \rightarrow \infty$, and $Y_{\cdot \wedge \tau_{n}}^{p}$ decreasingly converges to $Y \cdot \wedge \tau_{n}=v\left(\cdot, X^{\tau_{n}}\right)$, for some function $v$, as $p \rightarrow \infty$. The convergence of $Y_{\cdot \wedge \tau_{n}}^{m, p}$ to $Y_{\cdot \wedge \tau_{n}}$ is also uniform and $Z_{\cdot \wedge \tau_{n}}^{m, p}$ also converges to some $Z$. $\wedge \tau_{n}$ in bmo; cf. [5], Theorems 4.5 and 4.7. Sending $n \rightarrow \infty$, we obtain a solution to $(3.7)$.

4. Proof of Theorem 2.5. Within this proof, all the constants $T, d, N, \Lambda, L$, $\|b\|_{\mathbb{L}^{\infty}},\|\sigma\|_{\mathbb{L}^{\infty}}$, and functions $\left\{h_{n}\right\}$, which define the setting or appear in the assumptions of Theorem 2.5 will be thought of as global variables; any function of them will be treated as a constant, which we call an universal constant. For quantities dependent on additional parameters, we write, for example, $C=C(\psi)$ to signal that, in addition to the global variables mentioned above, $C$ also depends on $\psi$. In Hardy's manner, universal, constants will always be denoted by the letter $C$ which may change from line to line, and they are always positive. To increase readability, we use the notation $\leq_{C}$ as follows:

$$
\text { ' } a \leq_{C} b \text { ' stands for ' } a \leq C b \text { '. }
$$


Furthermore, we fix both $m$ and $n \in \mathbb{N}$, and removing them almost entirely from the notation throughout this section. It is important to note, however, that our treatment of $m$ and $n$ will be different. One one hand, since we are after uniform estimates on the entire sequence $\left\{\boldsymbol{v}^{m}\right\}$, we do not allow any of our constants to depend on $m$. (We will see later that the dependence on $\left\{k_{n}^{m}\right\}$ is through its $\mathbb{L}^{q_{n}}$-norm which is assumed to be bounded uniformly in $m$.) On the other hand, all of our analysis in this section will be restricted locally to the ball $B_{n}\left(b_{0}\right)$. Therefore, $n$ is be added, temporarily, to the list of universal constants and all the estimates below will depend on it implicitly. Hence, for the time being, the conditions of Theorem 2.5 is localized to $x \in B_{n}\left(b_{0}\right)$ and we simply assume, for the reminder of this section, that conditions of (1)-(4), with the center $b_{0}$ of the ball $B_{n}\left(b_{0}\right)$ and indices $m, n$ removed, are satisfied. In particular, we assume that there exists constants $\alpha, b, c, \ell, M>0$ and $q>1+d / 2$ such that

$$
\|\boldsymbol{v}\|_{\mathbb{L}^{\infty}\left([0, T] \times B_{n}\right)} \leq c \quad \text { and } \quad\|\boldsymbol{g}\|_{C^{\alpha}\left(B_{n}\right)} \leq b .
$$

There exists a Lyapunov pair $(h, k) \in \mathbf{L y}(\boldsymbol{f}, 2 c)$ on $B_{n}$ such that

$$
\|k\|_{\mathbb{L}^{q}\left([0, T] \times B_{n}\right)} \leq \ell \quad \text { and } \quad|\boldsymbol{f}(t, x, \boldsymbol{y}, \boldsymbol{z})| \leq M\left(|z|^{2}+k(t, x)\right),
$$

for all $(t, x) \in[0, T] \times B_{n},|\boldsymbol{y}| \leq c$, and $z \in \mathbb{R}^{N \times d}$. The $\alpha, b, c, \ell, M$ and $q$ are also added to the list of universal constants. Throughout this section, the dependence on $b_{0}$ is only through $\alpha, b, c, \ell, M$ and $q$.

4.1. A "testing" lemma. As we already mentioned, for each initial condition, the $\operatorname{SDE}$ (2.1) admits a unique strong solution $X^{t, x}=\left(X_{u}^{t, x}\right)_{u \in[t, T]}$. For notational convenience in several proofs below, we allow $X$ to start from negative time, that is, $t \leq 0$. Therefore, we extend $b$ and $\sigma$ via

$$
b(t, x)=b(0, x) \quad \text { and } \quad \sigma(t, x)=\sigma(0, x) \quad \text { for } t \leq 0 .
$$

These extended coefficients still satisfy conditions (1)-(3) after (2.1), ensuring the existence of the unique strong solution, which is still denoted by $X^{t, x}=$ $\left(X_{u}^{t, x}\right)_{u \in[t, T]}$. Its infinitesimal generator is given by

$$
\mathcal{L}=\sum_{i=1}^{d} b_{i}(\cdot, \cdot) D_{i}+\frac{1}{2} \sum_{i, j=1}^{d} a(\cdot, \cdot) D_{i j} .
$$

Parametrized by $(t, x)$, the laws of these solutions constitute a Markov family $\left(\mathbb{P}^{t, x}\right)_{(t, x) \in(-\infty, T] \times \mathbb{R}^{d}}$ of probability measures on $C\left([0, T] \rightarrow \mathbb{R}^{d}\right)$. (Even through the canonical process may start from negative time, we only focus on its trajectory on $[0, T]$.) In a minimal notational overload, we use $X$ for the coordinate map on $C\left([0, T] \rightarrow \mathbb{R}^{d}\right)$ and set throughout

$$
\boldsymbol{Y}_{u}=\boldsymbol{v}\left(u, X_{u}\right) \quad \text { and } \quad \boldsymbol{Z}_{u}=\boldsymbol{w}\left(u, X_{u}\right) \quad \text { for } u \in[t \vee 0, T] .
$$


A $C^{1,2}$-function $\varphi:[0, T] \times \mathbb{R}^{d} \rightarrow[0,1]$ is said to be testable if its support is contained in $[0, T] \times B_{n}$ and we have $\Gamma_{\varphi}<\infty$, where

$$
\Gamma_{\varphi}:=\sup _{(t, x): \varphi(t, x)>0}\left(\left|\frac{\partial}{\partial t} \varphi\right|+|D \varphi b|+\frac{1}{2}\left|D^{2} \varphi: a\right|+\frac{1}{\varphi}|D \varphi|^{2}\right) .
$$

LEMMA 4.1. With $c$ as in (4.1), there exists a universal constant $C>0$ such that, for each $\boldsymbol{c} \in \mathbb{R}^{N}$ with $|\boldsymbol{c}| \leq c$, all $t^{\prime} \in[t \vee 0, T], x \in \mathbb{R}^{d}$, and any testable $\varphi$, we have

$$
\begin{aligned}
\mathbb{E}^{t, x}\left[\int_{t^{\prime}}^{T} \mathbf{1}_{\left\{\varphi\left(u, X_{u}\right)=1\right\}}\left|\boldsymbol{Z}_{u}\right|^{2} d u\right] \\
\quad \leq \Gamma_{\varphi} \Gamma^{t, x}\left[\int_{t^{\prime}}^{T} \mathbf{1}_{\left\{\varphi\left(u, X_{u}\right) \in(0,1)\right\}}\left|\boldsymbol{Y}_{u}-\boldsymbol{c}\right|^{2} d u\right]+ \\
\quad+\mathbb{E}^{t, x}\left[\int_{t^{\prime}}^{T} \mathbf{1}_{\left\{\varphi\left(u, X_{u}\right)>0\right\}} k\left(u, X_{u}\right) d u\right]+\mathbb{E}^{t, x}\left[\mathbf{1}_{\left\{\varphi\left(T, X_{T}\right)>0\right\}}\left|\boldsymbol{Y}_{T}-\boldsymbol{c}\right|^{2}\right],
\end{aligned}
$$

where the expectation $\mathbb{E}^{t, x}$ is with respect to $\mathbb{P}^{t, x}$.

PROOF. We overload the notation by writing $\varphi$ for both the process $\varphi(\cdot, X)$ and the function $\varphi$; similarly, having fixed $\boldsymbol{c} \in \mathbb{R}^{N}$ with $|\boldsymbol{c}| \leq c$, we write $h^{c}$ both for the function $h(\cdot-\boldsymbol{c})$ and the process $h(\boldsymbol{Y}-\boldsymbol{c})$. We define the product process $F=\varphi h^{c}=\varphi(\cdot, X) h(\boldsymbol{Y}-\boldsymbol{c})$ and write down the semimartingale decompositions (under any $\mathbb{P}^{t, x}$ ):

$$
d \varphi=\left(\frac{\partial}{\partial u} \varphi+\mathcal{L} \varphi\right) d u+D \varphi \sigma d W
$$

and

$$
d h^{c}=\left(\frac{1}{2} D^{2} h^{c}:\langle\boldsymbol{Z}, \boldsymbol{Z}\rangle_{a}-D h^{c} \boldsymbol{f}\right) d u+D h^{c} \boldsymbol{Z} \sigma d W .
$$

Reminding the reader that $d F \stackrel{m}{=} \alpha$ means that $F-\int_{0}^{\cdot} \alpha_{s} d s$ is a local martingale, we conclude that

$$
d F \stackrel{m}{=} \varphi\left(\frac{1}{2} D^{2} h^{c}:\langle\boldsymbol{Z}, \boldsymbol{Z}\rangle_{a}-D h^{c} \boldsymbol{f}\right)+h^{c}\left(\frac{\partial}{\partial u} \varphi+\mathcal{L} \varphi\right)+\left\langle D h^{c} \boldsymbol{Z}, D \varphi\right\rangle_{a} .
$$

The $C^{2}$-regularity of the function $h$ and the fact that $h(\mathbf{0})=D h(\mathbf{0})=\mathbf{0}$ imply that there exists a constant $C>0$ which depends only on $h$ and $c$ such that

(4.7) $h^{c}(\boldsymbol{y}) \leq C|\boldsymbol{y}-\boldsymbol{c}|^{2} \quad$ and $\quad\left|D h^{c}(\boldsymbol{y})\right|^{2} \leq C|\boldsymbol{y}-\boldsymbol{c}|^{2} \quad$ for all $\boldsymbol{y}$ with $|\boldsymbol{y}| \leq c$.

The fact that $(h, k) \in \mathbf{L y}(\boldsymbol{f}, 2 c)$ coupled with the boundedness of $a$ and the fact that $|\boldsymbol{Y}-\boldsymbol{c}| \leq 2 c$, imply that the right-hand side of (4.6) above is bounded from 
below by

$$
\begin{gathered}
\varphi\left(|\boldsymbol{Z}|^{2}-k\right)-C|\boldsymbol{Y}-\boldsymbol{c}|^{2}\left|\frac{\partial}{\partial t} \varphi+\mathcal{L} \varphi\right|-\sum_{i l}\left(\frac{1}{2} \varphi\left(Z_{i l}\right)^{2}+C \frac{1}{2 \varphi}\left(D_{i} h^{c} D_{l} \varphi\right)^{2}\right) \mathbf{1}_{\varphi>0} \\
\geq \frac{1}{2} \varphi|\boldsymbol{Z}|^{2}-\varphi k-C|\boldsymbol{Y}-\boldsymbol{c}|^{2}\left(\left|\frac{\partial}{\partial u} \varphi+\mathcal{L} \varphi\right|+\frac{1}{\varphi}|D \varphi|^{2}\right) \mathbf{1}_{\varphi>0 .}
\end{gathered}
$$

It remains take the expectation and use boundedness of $F$ (implied by the boundedness of $\varphi$ and $\boldsymbol{Y}$ on $[0, T] \times B_{n}$, as well as continuity of $h^{c}$ ) and its positivity.

4.2. First consequences of the regularity of transition densities. It follows from the conditions imposed on $\sigma$ and $b$ (see [52], Theorem 3.2.1, page 71) that the Markov family $\left(\mathbb{P}^{t, x}\right)$ admits a family of transition densities

$$
p\left(t, x ; t^{\prime}, x^{\prime}\right), \quad t^{\prime} \in[t, T], x^{\prime} \in \mathbb{R}^{d} .
$$

Moreover, they satisfy the following fundamental estimate (known as the Aronson's estimate): there exist constants $\underline{\sigma}, \bar{\sigma}>0$, as well as $\underline{C}, \bar{C}>0$, depending only on the $\mathbb{L}^{\infty}$ - and ellipticity bounds on $b$ and $\sigma$, such that, for all $0 \leq t<t^{\prime} \leq T$ and all $x, x^{\prime} \in \mathbb{R}^{d}$, we have

$$
\begin{aligned}
\frac{\underline{C}}{\left(t-t^{\prime}\right)^{d / 2}} e^{-\frac{r}{2 \underline{\sigma}^{2}}} & \leq p\left(t, x ; t^{\prime}, x^{\prime}\right) \\
& \leq \frac{\bar{C}}{\left(t-t^{\prime}\right)^{d / 2}} e^{-\frac{r}{2 \bar{\sigma}^{2}}} \quad \text { where } r=\frac{\left|x^{\prime}-x\right|^{2}}{t^{\prime}-t} .
\end{aligned}
$$

REMARK 4.2. Under our assumptions [cf. conditions (1)-(3) after (2.1)], the upper bound in (4.8) can be obtained by the parametrix method (see [34], equation (6.12), page 24). The lower bound was first obtained in the paraboloid $\left|x^{\prime}-x\right|^{2} \leq \operatorname{const}\left(t^{\prime}-t\right)$ by [38], equation (4.75), then extended globally by a standard chaining argument. When $b$ and $\sigma$ are only measurable and $\mathcal{L}$ is in the divergence form, (4.8) was obtained by [1], Theorem 1.

The first consequence of the estimates (4.8) is the following uniform boundedness result.

LEMma 4.3. There exists a universal constant $C>0$ such that

$$
\mathbb{E}^{t, x}\left[\int_{t}^{T}\left(\left|\boldsymbol{Z}_{u}\right|^{2}+k\left(u, X_{u}\right)\right) \mathbf{1}_{\left\{X_{u} \in B_{n}\right\}} d u\right] \leq C,
$$

for all $t, x \in[0, T] \times \mathbb{R}^{d}$. 
PROOF. Let $\chi$ be a testable function, such that, for all $t \in[0, T], \chi(t, x)=1$ for $x \in B_{n-1}$ and $\chi(t, x)=0$ for $x \in B_{n}^{c}$. According to Lemma 4.1 and the boundedness of $\boldsymbol{v}$ on $[0, T] \times B_{n}$, there exists a universal constant $C=C(\chi)$ such that, uniformly over $(t, x) \in[0, T] \times \mathbb{R}^{d}$ we have

$$
\mathbb{E}^{t, x}\left[\int_{t}^{T}\left|\boldsymbol{Z}_{u}\right|^{2} \mathbf{1}_{\left\{X_{u} \in B_{n-1}\right\}} d u\right] \leq_{C} 1+\mathbb{E}^{t, x}\left[\int_{t}^{T} k\left(u, X_{u}\right) \mathbf{1}_{\left\{X_{u} \in B_{n}\right\}} d u\right] .
$$

By Hölder's inequality with $1 / q+1 / q^{\prime}=1$, we have

$$
\begin{aligned}
\mathbb{E}^{t, x}[ & \left.\int_{t}^{T} k\left(u, X_{u}\right) \mathbf{1}_{\left\{X_{u} \in B_{n}\right\}} d u\right] \\
& =\int_{t}^{T} \int_{B_{n}} k(u, \xi) p(t, x, u ; \xi) d u d \xi \\
& \leq\|k\|_{\mathbb{L}^{q}\left([0, T] \times B_{n}\right)}\left(\int_{t}^{T} \int_{B_{n}} p(t, x, u ; \xi)^{q^{\prime}} d u d \xi\right)^{1 / q^{\prime}} .
\end{aligned}
$$

The proof is completed once we use the upper bound in (4.8) and the fact that $q^{\prime}<1+2 / d$ to obtain

$$
\begin{aligned}
& \int_{t}^{T} \int_{B_{n}} p(t, x ; u, \xi)^{q^{\prime}} d u d \xi \\
& \quad \leq C \int_{t}^{T}(u-t)^{-\frac{d}{2}\left(q^{\prime}-1\right)}\left(\int_{B_{n}}(u-t)^{-\frac{d}{2}} e^{-\frac{q^{\prime}|\xi-x|^{2}}{2 \bar{\sigma}^{2}(u-t)}} d \xi\right) d u \\
& \quad \leq C \int_{t}^{T}(u-t)^{-\frac{d}{2}\left(q^{\prime}-1\right)} d u \\
& \quad \leq T^{1-\frac{d}{2(q-1)}} .
\end{aligned}
$$

The uniform bound of Lemma 4.3 helps provide the following fundamental relation between $\boldsymbol{w}$ and $\boldsymbol{v}$.

LEMMA 4.4. $\boldsymbol{w}$ is the weak (spatial) Jacobian Dv of $\boldsymbol{v}$ on $(0, T) \times \mathbb{R}^{d}$.

Proof. Given $\epsilon \in(0, T / 2)$ and the testable function $\chi$ from the proof of Lemma 4.3, we define

$$
\hat{\boldsymbol{v}}(t, x)=\boldsymbol{v}(t, x) \chi(t, x) \quad \text { for } t, x \in[0, T] \times \mathbb{R}^{d},
$$

and let the sequence $\left\{\hat{\boldsymbol{v}}^{(l)}\right\}$ (with $l \geq 1 / \epsilon$ ) of approximations to $\hat{\boldsymbol{v}}$ be given by

$$
\hat{\boldsymbol{v}}^{(l)}(t, x)=l \int_{t}^{t+1 / l} \mathbb{E}^{t, x}\left[\hat{\boldsymbol{v}}\left(u, X_{u}\right)\right] d u \quad \text { for } t, x \in[0, T-\epsilon] \times \mathbb{R}^{d} .
$$

The functions $\left(\hat{\boldsymbol{v}}^{(l)}\right)$ are uniformly bounded (by $c$, in fact), and, thanks to smoothness of the transition densities of $X$, each $\hat{\boldsymbol{v}}^{(l)}$ is $C^{1,2}$-differentiable. Moreover, as 
one readily checks, we have

$$
\left(\frac{d}{d t}+\mathcal{L}\right) \hat{\boldsymbol{v}}^{(l)}(t, x)=l\left(\mathbb{E}^{t, x}\left[\hat{\boldsymbol{v}}\left(t+1 / l, X_{t+1 / l}\right)\right]-\hat{\boldsymbol{v}}(t, x)\right) .
$$

Having fixed a pair $(t, x) \in[0, T-\epsilon] \times \mathbb{R}^{d}$, we apply Itô's formula to $\hat{\boldsymbol{v}}\left(\cdot, X^{t, x}\right)$, use the boundedness of $v$ on $[0, T] \times B_{n}$ in the second inequality below, and recall the second inequality in (4.2) in the last inequality, to obtain

$$
\begin{aligned}
\left|\left(\frac{d}{d t}+\mathcal{L}\right) \hat{\boldsymbol{v}}^{(l)}(t, x)\right| \leq_{C} l \mathbb{E}^{t, x}\left[\int_{t}^{t+1 / l}\left|\boldsymbol{v}\left(u, X_{u}\right)\right|\left(\frac{d}{d t}+\mathcal{L}\right) \chi\left(u, X_{u}\right) \mid d u\right] \\
+l \mathbb{E}^{t, x}\left[\int_{t}^{t+1 / l} \chi\left(u, X_{u}\right)\left|\boldsymbol{f}\left(u, X_{u}, \boldsymbol{Y}_{u}, \boldsymbol{Z}_{u}\right)\right| d u\right] \\
\quad+l \mathbb{E}^{t, x}\left[\int_{t}^{t+1 / l}\left|D \chi\left(u, X_{u}\right)\right|\left|\boldsymbol{Z}_{u}\right| d u\right] \\
\leq_{C} 1+l \mathbb{E}^{t, x}\left[\int_{t}^{t+1 / l} \chi\left(u, X_{u}\right)\left|\boldsymbol{f}\left(u, X_{u}, \boldsymbol{Y}_{u}, \boldsymbol{Z}_{u}\right)\right| d u\right] \\
\quad+\mathbb{E}^{t, x}\left[\int_{t}^{t+1 / l} \mathbf{1}_{\left\{\chi\left(u, X_{u}\right)>0\right\}}\left|\boldsymbol{Z}_{u}\right|^{2} d u\right] \\
\leq_{C} 1+l \mathbb{E}^{t, x}\left[\int_{t}^{t+1 / l}\left(k\left(u, X_{u}\right)+\left|\boldsymbol{Z}_{u}\right|^{2}\right) \mathbf{1}_{\left\{X_{u} \in B_{n}\right\}} d u\right],
\end{aligned}
$$

for a universal constant $C=C(\chi)$. The Markov property of the family $\left(\mathbb{P}^{t, x}\right)$ now implies that, with $\boldsymbol{Z}_{s}^{\prime}=\boldsymbol{w}\left(s, X_{s}^{\prime}\right)$, where $X^{\prime}$ denotes the coordinate process inside the $\mathbb{P}^{u, X_{u}}$-expectation, we have

$$
\begin{aligned}
\mathbb{E}^{t, x} & {\left[\int_{t}^{T-\epsilon}\left|\left(\frac{d}{d t}+\mathcal{L}\right) \hat{\boldsymbol{v}}^{(l)}\left(u, X_{u}\right)\right| d u\right] } \\
& \leq C 1+\mathbb{E}^{t, x}\left[\int_{t}^{T-\epsilon} \mathbb{E}^{u, X_{u}}\left[l \int_{u}^{u+1 / l}\left(\left|\boldsymbol{Z}_{s}^{\prime}\right|^{2}+k\left(s, X_{s}^{\prime}\right) \mathbf{1}_{\left\{X_{s}^{\prime} \in B_{n}\right\}}\right) d s\right] d u\right] \\
& =1+\mathbb{E}^{t, x}\left[\int_{t}^{T-\epsilon} l \int_{u}^{u+1 / l}\left(\left|\boldsymbol{Z}_{s}\right|^{2}+k\left(s, X_{s}\right)\right) \mathbf{1}_{\left\{X_{s} \in B_{n}\right\}} d s d u\right] \\
& \leq C \\
& 1+\mathbb{E}^{t, x}\left[\int_{t}^{T-\epsilon}\left(\left|\boldsymbol{Z}_{s}\right|^{2}+k\left(s, X_{s}\right)\right) \mathbf{1}_{\left\{X_{s} \in B_{n}\right\}} d s\right] \leq C \quad \text { for all } \ell,
\end{aligned}
$$

where the last inequality follows from Lemma 4.3; the constant $C$ obtained above is also uniform for all $t, x \in[0, T-\epsilon] \times \mathbb{R}^{d}$.

Continuity of $v$ implies that $\hat{v}$ is also continuous, and hence, uniformly continuous on compacts. For any $\tilde{\epsilon}>0$, there exists $\delta=\delta(\tilde{\epsilon}, n) \in(0,1)$ such that

$$
\left|\hat{\boldsymbol{v}}(t, x)-\hat{\boldsymbol{v}}\left(t^{\prime}, x^{\prime}\right)\right| \leq \tilde{\epsilon} \quad \text { if } \quad\left|(t, x)-\left(t^{\prime}, x^{\prime}\right)\right| \leq \delta \text { and }\left|x-b_{0}\right| \leq n+1 .
$$

The difference above vanishes if $\left|(t, x)-\left(t^{\prime}, x^{\prime}\right)\right| \leq \delta$ and $\left|x-b_{0}\right|>n+1$, since, in that case, $x, x^{\prime} \notin \operatorname{supp} \chi$. Therefore, using the boundedness of $\hat{v}$ and the upper 
bound in (4.8), for $(t, x) \in[0, T-\epsilon] \times \mathbb{R}^{d}$ we have

$$
\begin{aligned}
\left|\hat{\boldsymbol{v}}^{(l)}(t, x)-\hat{\boldsymbol{v}}(t, x)\right| & \\
\leq & \iint_{[t, t+1 / l] \times \mathbb{R}^{d}} \mathbf{1}_{\{|\xi-x|>\delta\}}|\hat{\boldsymbol{v}}(u, \xi)-\hat{\boldsymbol{v}}(t, x)| l p(t, x ; u, \xi) d u d \xi \\
& +\iint_{[t, t+1 / l] \times \mathbb{R}^{d}} \mathbf{1}_{\{|\xi-x| \leq \delta,|x| \leq n+1\}}|\hat{\boldsymbol{v}}(u, \xi)-\hat{\boldsymbol{v}}(t, x)| \operatorname{lp}(t, x ; u, \xi) d u d \xi \\
\leq & l \int_{0}^{1 / l} \int_{|\xi| \geq \delta} u^{-d / 2} e^{-\frac{|\xi|^{2}}{2 \bar{\sigma}^{2} u}} d \xi d u+\tilde{\epsilon} \\
\leq & l \int_{0}^{1 / l}\left(1-\Phi\left(\delta / \sqrt{\bar{\sigma}^{2} u}\right)\right) d u+\tilde{\epsilon},
\end{aligned}
$$

where $\Phi(\cdot)$ is the distribution function for standard normal. Note that $\lim _{u \downarrow 0} \Phi\left(\delta / \sqrt{\bar{\sigma}^{2} u}\right)=1$. The last expression is less than $2 \tilde{\epsilon}$, for sufficiently large $l$, uniformly for $t, x \in[0, T-\epsilon] \times \mathbb{R}^{d}$. Since the choice of $\tilde{\epsilon}$ is arbitrary, the previous estimates implies the uniform convergence of $\left\{\hat{\boldsymbol{v}}^{(l)}\right\}$ to $\hat{\boldsymbol{v}}$ on $[0, T-\epsilon] \times \mathbb{R}^{d}$.

Setting $\hat{\boldsymbol{Y}}^{(l)}=\hat{\boldsymbol{v}}^{(l)}\left(\cdot, X^{0, x}\right)$ and $\hat{\boldsymbol{Y}}=\hat{\boldsymbol{v}}\left(\cdot, X^{0, x}\right)$, we use the uniform convergence of $\left\{\hat{\boldsymbol{v}}^{(l)}\right\}$ to obtain $\hat{\boldsymbol{Y}}^{(l)} \rightarrow \hat{\boldsymbol{Y}}$, uniformly. Applying Itô's formula to $\left|\hat{\boldsymbol{Y}}-\hat{\boldsymbol{Y}}^{(l)}\right|^{2}$ and using (4.9), we obtain

$$
\mathbb{E}^{0, x}\left[\left\langle\hat{\boldsymbol{Y}}^{(l)}-\hat{\boldsymbol{Y}}\right\rangle_{T-\epsilon}\right] \leq C\left\|\sup _{u \in[0, T-\epsilon]}\left|\hat{\boldsymbol{Y}}_{u}^{(l)}-\hat{\boldsymbol{Y}}_{u}\right|\right\|_{\mathbb{L}^{\infty}},
$$

which converges to 0 . This means that

$$
\lim _{l} \int_{0}^{T-\epsilon} \int_{B_{n}} g_{l}^{2}(u, \xi) p(0, x ; u, \xi) d u d \xi=0,
$$

where

$$
g_{l}=\left|\left(\chi \boldsymbol{w}+\boldsymbol{v} D \chi-D \hat{\boldsymbol{v}}^{(l)}\right) \sigma\right| .
$$

By the lower bound in (4.8), the density $p(0, x ; \cdot, \cdot)$ is bounded away from 0 on $[\epsilon, T-\epsilon] \times B_{n}$ and $\sigma$ is uniformly elliptic. Therefore, $D \hat{\boldsymbol{v}}^{(l)} \rightarrow \chi \boldsymbol{w}+\boldsymbol{v} D \chi$ in $\mathbb{L}^{2}\left([\epsilon, T-\epsilon] \times B_{n}\right)$, as $l \rightarrow \infty$. Coupled with the fact that $\hat{\boldsymbol{v}}^{(l)} \rightarrow \hat{\boldsymbol{v}}$ also in $\mathbb{L}^{2}\left([\epsilon, T-\epsilon] \times B_{n}\right)$, this implies that $\chi \boldsymbol{w}+\boldsymbol{v} D \chi$ is the weak Jacobian of $\boldsymbol{v} \chi$. The statement follows by noting that for each compact $K \subseteq \mathbb{R}^{d}, \chi \boldsymbol{w}+\boldsymbol{v} D \chi=\boldsymbol{w}$ and $\boldsymbol{v} \chi=\boldsymbol{v}$ on $[0, T] \times K$, for large enough $n$, and the choice of $\epsilon$ is arbitrary.

4.3. Uniform local estimates. We now choose and fix $R \in(0,1 / 4]$ and a pair $\left(t_{0}, x_{0}\right) \in[0, T] \times B_{n-1}$. It is important to note that none of the constants in the sequence of lemmas in the next two subsections depends on the choice of $\left(t_{0}, x_{0}\right)$ and $R$. 
We will use the point $\left(t_{0}, x_{0}\right)$ (or only $\left.x_{0}\right)$ as the origin throughout the proof and dilate the coordinate system around it with the change of variables $(t, x) \rightarrow(\tau, \xi)$, given by

$$
x=x_{0}+R \xi \quad \text { and } \quad t=t_{0}+R^{2} \tau .
$$

Most balls, cylinders, etc. in the sequel will be centered around $\left(t_{0}, x_{0}\right)$ (or $x_{0}$ ) and their dimensions (radius, etc.) will have much nicer expressions in the $(\tau, \xi)$ coordinates, so we introduce the following notation:

$$
\begin{aligned}
\beta(\rho) & =\left\{x \in \mathbb{R}^{d}:\left|x-x_{0}\right| \leq \rho R\right\} \quad \text { and } \\
\gamma(\theta ; \rho) & =\left\{(t, x) \in[0, T] \times \mathbb{R}^{d}: t_{0} \leq t \leq t_{0}+\theta R^{2},\left|x-x_{0}\right| \leq \rho R\right\},
\end{aligned}
$$

for the ball $\beta$ and the parabolic cylinder $\gamma$.

Typically, a function $\tilde{\varphi}: \mathbb{R} \times \mathbb{R}^{d} \rightarrow \mathbb{R}$ will be defined in $(\tau, \xi)$-coordinates, and then its counterpart

$$
\varphi(t, x)=\tilde{\varphi}\left(\frac{t-t_{0}}{R^{2}}, \frac{x-x_{0}}{R}\right),
$$

restricted to $[0, T] \times \mathbb{R}^{d}$, is used in computations. A similar notation will be used for functions of $x$ only or for subsets of $[0, T] \times \mathbb{R}^{d}$ or $\mathbb{R}^{d}$ (identified with their indicators). In the same spirit, we set $\tilde{T}=\left(T-t_{0}\right) /\left(R^{2}\right)$.

Within this proof, $\int$ denotes the integral over $R^{d}$, while $\iint$ stands for the integral over $\left[t_{0}, T\right] \times \mathbb{R}^{d}$. If the domain of integration is notationally further restricted, as for example, in $\iint_{D}$, the integral is taken over $\left(\left[t_{0}, T\right] \times \mathbb{R}^{d}\right) \cap D$ (or $\mathbb{R}^{d} \cap D$ in the spatial case). Similarly, in order to avoid repeated explicit minimization with $T$, we assume that temporal variables cannot take values above $T$, so that, for example, the interval $\left[t_{0}, t_{0}+4 R^{2}\right]$ coincides with $\left[t_{0}, T\right]$, when $t_{0}+4 R^{2}>T$. Meanwhile, as we mentioned before, $t$ in $\mathbb{P}^{t, x}$ is allowed to be negative.

We continue with some consequences of (4.8) which will be used in the sequel. Given the origin $\left(t_{0}, x_{0}\right)$ and the radius $R$ fixed above, we introduce the following shortcut:

$$
p_{\varepsilon}(t, x)=p\left(t_{0}-\varepsilon R^{2}, x_{0} ; t, x\right) \quad \text { for } \varepsilon \geq 0,
$$

for the transition density, and state several useful estimates where the functions $\Delta_{\sigma}, \delta_{\sigma}$ are given by

$$
\Delta_{\sigma}(\varepsilon)=\exp \left(-\frac{1}{2 \sigma^{2}} \varepsilon^{-1}\right) \quad \text { and } \quad \delta_{\sigma}(\varepsilon)=\varepsilon^{-d / 2} \Delta_{\sigma}(\varepsilon)
$$

and extended to $\varepsilon=0, \varepsilon=+\infty$ by continuity. We also define the positive (universal) constant $\varepsilon_{0}$ by

$$
\varepsilon_{0}=\inf \left\{\varepsilon>0: \delta_{\bar{\sigma}}(\varepsilon)=1 / 2\right\} \wedge \min \left(1,\left(\bar{\sigma}^{2} d\right)^{-1}\right) .
$$

Note that $\delta_{\bar{\sigma}}(\epsilon)$ is increasing on $\left[0,\left(\bar{\sigma}^{2} d\right)^{-1}\right]$ and decreasing on $\left[\left(\bar{\sigma}^{2} d\right)^{-1}, \infty\right)$ with $\delta_{\bar{\sigma}}(0)=0$. Therefore, the definition of $\epsilon_{0}$ implies $\delta_{\bar{\sigma}}(\epsilon) \leq \delta_{\bar{\sigma}}\left(\epsilon_{0}\right) \leq 1 / 2$ for $\epsilon \in\left[0, \epsilon_{0}\right]$. The role of $\epsilon_{0}$ will be clear in Proposition 4.16 below. 
LEMMA 4.5. There exists a universal constant $C>0$ such that, for all $(t, x) \in$ $\gamma(4,4)$ and $\varepsilon \in\left(0, \varepsilon_{0} / 2\right]$, we have

(4.14) $p_{\varepsilon}(t, x) \leq_{C} R^{-d} \quad$ when $(t, x) \notin \gamma(1 / 4 ; 1 / 2)$,

(4.15) $p_{\varepsilon}(t, x) \geq_{C} R^{-d} \delta_{\underline{\sigma} / 4}\left(\varepsilon_{0} / 2+\varepsilon\right) \quad$ when $t \geq t_{0}+\varepsilon_{0} R^{2} / 2$,

(4.16) $p_{\varepsilon}(t, x) \leq_{C} R^{-d} \delta_{\bar{\sigma}}\left(\varepsilon_{0} / 2+\varepsilon\right) \quad$ when $t<t_{0}+\varepsilon_{0} R^{2} / 2$ and $x \notin \beta(1)$.

Proof. We set $\bar{\tau}=\varepsilon+\left(t-t_{0}\right) / R^{2}>0, \xi=\left(x-x_{0}\right) / R$ and $r=\bar{\tau} /|\xi|^{2} \in$ $(0, \infty]$, and note that the bounds in the density estimates (4.8) can be represented in two forms (with the outer one holding only for $\xi \neq 0$ ):

$$
|\xi|^{-d} \delta_{\underline{\sigma}}(r)=\bar{\tau}^{-\frac{d}{2}} \Delta_{\underline{\sigma}}(r) \leq_{C} R^{d} p_{\varepsilon}(t, x) \leq_{C} \bar{\tau}^{-\frac{d}{2}} \Delta_{\bar{\sigma}}(r)=|\xi|^{-d} \delta_{\bar{\sigma}}(r),
$$

which will be used throughout the proof.

- Inequality (4.14): Both $\delta_{\sigma}$ and $\Delta_{\sigma}$ are bounded by a constant $C$ on $[0, \infty]$, so, by the right-hand side of (4.17), we have

$$
R^{d} p_{\varepsilon}(t, x) \leq C \max (|\xi|, \sqrt{\bar{\tau}})^{-d}
$$

which, in turn, implies (4.14) since $\max (|\xi|, \sqrt{\bar{\tau}}) \geq 1 / 2$ on $\gamma(1 / 4 ; 1 / 2)^{c}$.

- Inequality (4.15): Under the conditions of (4.15), we have $r \geq \bar{\tau} / 16$, and so, by monotonicity of $\Delta_{\sigma}$ and (4.17), we have

$$
R^{d} p_{\varepsilon}(t, x) \geq_{C} \bar{\tau}^{-d / 2} \Delta_{\underline{\sigma}}(r) \geq_{C} \delta_{\underline{\sigma}}(\bar{\tau} / 16) \geq_{C} \delta_{\underline{\sigma} / 4}(\bar{\tau}) .
$$

The function $\delta_{\sigma / 4}$ attains it maximum at $16\left(\sigma^{2} d\right)^{-1}$, and is nondecreasing to the left of it and nonincreasing and positive to the right. Since $\varepsilon_{0} / 2+\varepsilon \leq \bar{\tau}$, in the case that $\bar{\tau} \leq 16\left(\underline{\sigma}^{2} d\right)^{-1}$, we have $\delta_{\underline{\sigma} / 4}(\bar{\tau}) \geq \delta_{\underline{\sigma} / 4}\left(\varepsilon_{0} / 2+\varepsilon\right)$. On the other hand, if $\bar{\tau} \in\left(16\left(\underline{\sigma}^{2} d\right)^{-1}, 4 \frac{1}{2}\right]$, we have

$$
\delta_{\underline{\sigma} / 4}\left(\varepsilon_{0} / 2+\varepsilon\right) \leq \delta_{\underline{\sigma} / 4}\left(16\left(\underline{\sigma}^{2} d\right)^{-1}\right) \leq{ }_{C} \delta_{\underline{\sigma} / 4}\left(4 \frac{1}{2}\right) \leq \delta_{\underline{\sigma} / 4}(\bar{\tau}) .
$$

Both alternatives lead to (4.15).

- Inequality (4.16): The conditions of (4.16) translate into $\bar{\tau} \leq \varepsilon_{0} / 2+\varepsilon \leq$ $\left(\bar{\sigma}^{2} d\right)^{-1}$, as well as $r \leq \bar{\tau}$ (since $|\xi| \geq 1$ ). The function $\delta_{\bar{\sigma}}$ is nondecreasing on $\left[0,\left(\bar{\sigma}^{2} d\right)^{-1}\right]$, so the (4.17) implies

$$
R^{d} p_{\varepsilon}(t, x) \leq{ }_{C} \delta_{\bar{\sigma}}(r) \leq \delta_{\bar{\sigma}}\left(\varepsilon_{0} / 2+\varepsilon\right)
$$

An operational form of Lemma 4.1, stated in Proposition 4.7 below, employs a particular testing function $\varphi$, obtained via (4.10) from a function $\tilde{\varphi}: \mathbb{R} \times \mathbb{R}^{d} \rightarrow$ $[0,1]$ in the class $C^{1,2}\left(\mathbb{R} \times \mathbb{R}^{d}\right)$ satisfying

$$
\begin{array}{ll}
\tilde{\varphi}(\tau, \xi)=1 & \text { when } \tau \leq 1 \text { and }|\xi| \leq 1, \\
\tilde{\varphi}(\tau, \xi)=0 & \text { when } \tau \geq 4 \text { or }|\xi| \geq 2
\end{array}
$$


and $\tilde{\varphi}(\tau, \xi) \in(0,1)$ otherwise. By making sure that the $\tilde{\varphi}$ decreases fast enough (e.g., quadratically) toward its 0-level set, one can also guarantee the boundedness of $|D \tilde{\varphi}|^{2} / \tilde{\varphi}$. For the corresponding function $\varphi$, expressed in the original coordinates [as defined in (4.10)], we easily check that, relative to the set $\left[t_{0}, T\right] \times \mathbb{R}^{d}$,

$$
\{\varphi=1\}=\gamma(1 ; 1) \quad \text { and } \quad\{\varphi=0\}=\overline{\gamma(4 ; 2)^{c}},
$$

and that the quantity $\Gamma_{\varphi}$ is independent of the choice of $\left(t_{0}, x_{0}\right)$. Due to $R \leq 1 / 4$, we have $|D \varphi b| \leq_{C} \frac{1}{R^{2}}|D \tilde{\varphi} b|$. Therefore, $\Gamma_{\varphi}$ satisfies

$$
\Gamma_{\varphi} \leq_{C} \frac{1}{R^{2}} \Gamma_{\tilde{\varphi}} .
$$

Finally, the support of $\varphi(t, \cdot)$ is a subset of $B_{n}$. This follows from $x_{0} \in B_{n-1}$, $\operatorname{supp} \tilde{\varphi}(\tau, \cdot)=B_{2}$, and $R \leq 1 / 4$.

LEMMA 4.6. For $p_{\epsilon}$ defined in (4.11), there exists a universal constant $C>0$ such that, with $\frac{1}{q^{\prime}}=1-\frac{1}{q}$ we have

$$
\iint_{\gamma(4 ; 2)} k p_{\epsilon} \leq_{C}(2 R \wedge \sqrt{T})^{2-\frac{2+d}{q}} .
$$

ProOF. By (4.8), with $t_{\epsilon}=t_{0}-\varepsilon R^{2}$, we have

$$
\begin{aligned}
\iint_{\gamma(4 ; 2)} p_{\varepsilon}^{q^{\prime}} & \leq C \int_{\left[t_{0}, t_{0}+4 R^{2}\right]}\left(t-t_{\epsilon}\right)^{-\frac{d}{2}\left(q^{\prime}-1\right)}\left(\int_{\beta(2)}\left(t-t_{\epsilon}\right)^{-\frac{d}{2}} e^{-\frac{q^{\prime}\left|x-x_{0}\right|^{2}}{2 \bar{\sigma}^{2}\left(t-t_{\epsilon}\right)}} d x\right) d t \\
& \leq \int_{C} \int_{\left[t_{0}, t_{0}+4 R^{2}\right]}\left(t-t_{\epsilon}\right)^{-\frac{d}{2}\left(q^{\prime}-1\right)} d t \leq \int_{\left[t_{0}, t_{0}+4 R^{2}\right]}\left(t-t_{0}\right)^{-\frac{d}{2}\left(q^{\prime}-1\right)} d t \\
& \leq_{C}(2 R \wedge \sqrt{T})^{2-\frac{d}{q-1}} .
\end{aligned}
$$

The previous inequality, combined with Hölder's inequality in the form $\iint k p_{\epsilon} \leq$ $\|k\|_{\mathbb{L}^{q}}\left(\iint p_{\epsilon}^{q^{\prime}}\right)^{1 / q^{\prime}}$, establishes the statement.

Reminding the reader that the constant $c$ is defined in (4.1), we state the following result which is a combination of Lemma 4.1, applied with the testing function $\varphi$ introduced above, and Lemmas 4.4, 4.6, together with (4.18).

PROPOSITION 4.7. There exists a universal constant $C>0$ such that for all $\varepsilon \geq 0$ and all $\boldsymbol{c}$ with $|\boldsymbol{c}| \leq c$, we have

$$
\begin{aligned}
\iint_{\gamma(1 ; 1)}|D \boldsymbol{v}|^{2} p_{\varepsilon} \leq C & R^{-2} \iint_{\gamma(4 ; 2) \backslash \gamma(1 ; 1)}|\boldsymbol{v}-\boldsymbol{c}|^{2} p_{\varepsilon} \\
& +\mathbf{1}_{\{\tilde{T} \leq 4\}} \int_{\beta(2)}|\boldsymbol{g}-\boldsymbol{c}|^{2} p_{\varepsilon}(T, \cdot)+(2 R \wedge \sqrt{T})^{2-\frac{2+d}{q}}
\end{aligned}
$$


4.4. A weighted Poincaré inequality and Struwe's lemma. Next, we state a weighted Poincaré inequality in Lemma 4.8 below. Let $\tilde{D}$ be a Lipschitz domain (nonempty open connected set) in $\mathbb{R}^{d}$, and let $D$ be its translate/dilate as described around (4.10). Similarly, let $\tilde{\chi}$ be a weight function, that is, such that $\tilde{\chi} \in \mathbb{L}^{\infty}(\tilde{D})$ and $\int_{\tilde{D}} \tilde{\chi}>0$, and let $\chi$ be its translated/dilated version. Given a function $u \in$ $\mathbb{L}^{1}(D)$, we define its $\chi$-average

$$
\bar{u}_{D}^{\chi}=\frac{1}{\int_{D} \chi} \int_{D} u \chi .
$$

The special case $\chi=1$ is denoted simply by $\bar{u}_{D}$. For a vector-valued function $\boldsymbol{u}$, the same notation is used, but with averaging is applied component-wise. When the domain $D$ is omitted, it is assumed that $D=\operatorname{Int} \operatorname{supp} \chi$.

In keeping with the notational philosophy of the proof, $\tilde{D}$ and $\tilde{\chi}$ are thought of as prototypes, and $D$ and $\chi$ as the family of their homothetic copies, indexed by $t_{0}, x_{0}$, and $R$. As above, the main message behind our results below is that estimates can be made independently (or explicitly dependently) of those indices. Here, $\|\cdot\|$ denotes the $\mathbb{L}^{2}$-norm on $D$ and $H^{1}(D)$ the Sobolev space $W^{1,2}$ on $D$.

LEMMA 4.8. Given $\tilde{D}$ and $\tilde{\chi}$ as above, there exists a universal constant $C=$ $C(\tilde{D}, \tilde{\chi})$ such that for all $u \in H^{1}(D)$ we have

$$
\left\|u-\bar{u}_{D}^{\chi}\right\|^{2} \leq_{C} R^{2}\|D u\|^{2} .
$$

Proof. For $w \in \mathbb{L}^{2}(\tilde{D})$, by the Cauchy-Schwarz inequality, we have

$$
\begin{aligned}
\left\|w-\frac{1}{|\tilde{D}|} \int_{\tilde{D}} w\right\|\left\|\tilde{\chi}-\frac{1}{|\tilde{D}|} \int_{\tilde{D}} \tilde{\chi}\right\| & \geq\left|\int_{\tilde{D}} w \tilde{\chi}-\frac{1}{|\tilde{D}|} \int_{\tilde{D}} w \int_{\tilde{D}} \tilde{\chi}\right| \\
& =\left|\int_{\tilde{D}} \tilde{\chi}\right|\left|\bar{w}_{\tilde{D}}^{\tilde{\chi}}-\bar{w}_{\tilde{D}}\right| .
\end{aligned}
$$

If, additionally, $w \in H^{1}(\tilde{D})$, then combining the previous inequality and Poincaré's inequality, we have

$$
\left\|w-\bar{w}_{\tilde{D}}^{\tilde{\chi}}\right\|^{2} \leq 2\left(\bar{w}_{\tilde{D}}-\bar{w}_{\tilde{D}}^{\tilde{\chi}}\right)^{2}+2\left\|w-\bar{w}_{\tilde{D}}\right\|^{2} \leq C\left\|w-\bar{w}_{\tilde{D}}\right\|^{2} \leq C\|\nabla w\|^{2},
$$

with $C$ depending only on $\tilde{D}$ and $\tilde{\chi}$. It remains to set $w(\xi)=u\left(x_{0}+R \xi\right)$.

Next, let us pick a (weight function) $\tilde{\psi}: \mathbb{R}^{d} \rightarrow[0,1]$ such that

$$
\tilde{\psi}(\xi)=0 \quad \text { for }|\xi| \leq \frac{1}{2} \text { or }|\xi| \geq 4, \quad \tilde{\psi}(\xi)=1 \quad \text { for } 1 \leq|\xi| \leq 2
$$

and $\tilde{\psi}(\xi) \in(0,1)$, otherwise, and consider its version $\psi$ in the $x$-coordinates. 
LEMMA 4.9. When $d \geq 2$, there exists a universal constant $C=C(\tilde{\psi})$ such that, for any $u \in H_{\mathrm{loc}}^{1}\left(\mathbb{R}^{d}\right)$ we have

$$
\int_{\beta(2) \backslash \beta(1)}\left|u-\bar{u}^{\psi}\right|^{2} \leq_{C} R^{2} \int_{\beta(4) \backslash \beta(1 / 2)}|D u|^{2} .
$$

The same inequality holds when the domain of the left integral is replaced by $\beta(2)$ and that of right one is replaced by $\beta(4)$, but $d$ is allowed to be 1 in this case.

Proof. With $\tilde{D}$ such that $D=\beta(4) \backslash \beta(1 / 2)$, we observe that supp $\psi \subseteq D$ and that $\beta(2) \backslash \beta(1) \subseteq D$. Therefore, applying Lemma 4.8, we have

$$
\int_{\beta(2) \backslash \beta(1)}\left|u-\bar{u}^{\psi}\right|^{2} \leq \int_{D}\left|u-\bar{u}_{D}^{\psi}\right|^{2} \leq_{C} R^{2} \int_{D}|D u|^{2} .
$$

The proof is the same when $\beta(2) \backslash \beta(1)$ is replaced by $\beta(2)$ and $D=\beta(4)$.

REMARK 4.10. When $d=1$, the set $\beta(4) \backslash \beta(1 / 2)=\left[-4,-\frac{1}{2}\right] \cup\left[\frac{1}{2}, 4\right]$ is not connected and, in fact, the statement of Lemma 4.9 does not hold. To see that, it is enough to consider $u=1$ on $\left[-4,-\frac{1}{2}\right]$ and $u=2$ on $\left[4, \frac{1}{2}\right]$. Then $\int_{\beta(4) \backslash \beta(1 / 2)}|D u|^{2}=0$, but $1<\bar{u}^{\psi}<2$ implies $\int_{\beta(2) \backslash \beta(1)}\left|u-\bar{u}^{\psi}\right|^{2}>0$.

On the other hand, let us argue that we can assume, without loss of generality, that $d \geq 2$ in Theorem 2.5. Indeed, suppose that we have established Theorem 2.5 for $d \geq 2$, but we are facing a situation where $d=1$. In this case, we simply embed our one-dimensional problem into a two-dimensional one. More precisely, we define the new state process, perhaps on an enlarged probability space, as $\hat{X}=$ $(X, B)$, where $B$ is a Brownian motion independent of $W$. The so-obtained coefficients $\hat{b}=(b, 0)$ and $\hat{\sigma}=\operatorname{diag}(\sigma, 1)$ satisfy all the necessary assumptions. Furthermore, the functions $\hat{\boldsymbol{v}}^{m}\left(t, x, x^{\prime}\right)=\boldsymbol{v}^{m}(t, x)$ and $\hat{\boldsymbol{w}}^{m}\left(t, x, x^{\prime}\right)=\left(\boldsymbol{w}^{m}(t, x), \mathbf{0}\right)$ is a Markovian solution to the system (2.5) on the enlarged probability space. The similarly defined $\hat{\boldsymbol{f}}^{m}$ and $\hat{\boldsymbol{g}}^{m}$ satisfy the assumptions of Theorem 2.5. In particular, for the Lyapunov pair in condition (4), inequality (2.4) (with $f$ and $k_{n}$ replaced by $\boldsymbol{f}^{m}$ and $k_{n}^{m}$, resp.) is satisfied for all $\hat{z}=(\boldsymbol{z}, \mathbf{0}) \in \mathbb{R}^{N \times 2}$. Therefore, Theorem 2.5 implies that $\left\{\hat{\boldsymbol{v}}^{m}\right\}$ is uniformly locally Hölderian in its first two variables. As a result, Theorem 2.8 produces a locally Hölderian solution $(\hat{\boldsymbol{v}}, \hat{\boldsymbol{w}})$ on the extended space. It remains to use locally uniform convergence and Lemma 4.4 to conclude that $\hat{v}$ does not depend on the additional coordinate $x^{\prime}$ and that the second column of $\hat{\boldsymbol{w}}$ vanishes. Therefore, $\boldsymbol{Y}=\hat{\boldsymbol{v}}(\cdot, X, 0)$ and $\boldsymbol{Z}=\hat{\boldsymbol{w}}^{1}(\cdot, X, 0)$ are adapted to the original filtration and solves the original system.

We consider $\tilde{\psi}$ as a global variable for the remainder of the proof. Consequently, the dependence of universal constants on it will be suppressed in sequel. The following lemma generalizes an important result of Struwe (see [53], Lemma 4, page 134). 
LEMMA 4.11. There exists a universal constant $C$ such that

$$
\left|\overline{\boldsymbol{v}\left(t_{2}, \cdot\right)} \psi-\overline{\boldsymbol{v}\left(t_{1}, \cdot\right)} \psi\right|^{2} \leq_{C} R^{-d} \iint_{\gamma\left(\theta_{2}, 4\right) \backslash\left(\gamma\left(\theta_{1}, 4\right) \cup \gamma\left(\theta_{2}, 1 / 2\right)\right)}|D \boldsymbol{v}|^{2}+R^{2-\frac{2+d}{q}},
$$

for all $0 \leq \theta_{1} \leq \theta_{2} \leq 4$, where $t_{i}=t_{0}+\theta_{i} R^{2}, i=1,2$.

PROOF. We fix $\varepsilon \in(0,1]$, and, reminding the reader that $p_{\varepsilon}=p\left(t_{0}-\varepsilon R^{2}\right.$, $\left.x_{0}, \cdot, \cdot\right)$, set $\eta=\psi / p_{\varepsilon}$. Itô's formula and Lemma 4.4, applied to the product $F_{t}=$ $\eta\left(t, X_{t}\right) v^{i}\left(t, X_{t}\right)\left(\right.$ with $\left.X=X^{t_{0}-\varepsilon R^{2}, x_{0}}\right)$ yields

$$
d\left(\eta v^{i}\right) \stackrel{m}{=}-\eta f^{i}+v^{i}\left(\frac{\partial}{\partial t} \eta+\mathcal{L} \eta\right)+\left\langle D v^{i}, D \eta\right\rangle_{a}
$$

Therefore, with $L=\mathbb{E}^{\varepsilon}\left[F_{t_{2}}-F_{t_{1}}\right]=\left(\overline{v^{i}\left(t_{2}, \cdot\right)} \psi \overline{v^{i}\left(t_{1}, \cdot\right)} \psi\right) \int \psi$ and the understanding that all space-time integrals $\iint$ in the rest of the proof are over $\gamma\left(\theta_{2}, 4\right) \backslash \gamma\left(\theta_{1}, 4\right)$ we have

$$
L=-\iint f^{i} \psi+\iint\left(p_{\varepsilon} v^{i}\left(\frac{\partial}{\partial t} \eta+\mathcal{L} \eta\right)+p_{\epsilon}\left\langle D v^{i}, D \eta\right\rangle_{a}\right) .
$$

Since $\sigma$ is bounded and globally Lipschitz, so is $a$, and the infinitesimal generator $\mathcal{L}$ can be written in a divergence form

$$
\mathcal{L}=\sum_{k} \tilde{b}_{k} D_{k}+\frac{1}{2} \sum_{j, k} D_{j}\left(a_{j k} D_{k}\right)
$$

where $\tilde{b}_{k}=b_{k}-\frac{1}{2} \sum_{j} D_{j}\left(a_{j k}\right)$ is bounded and $D_{j}\left(a_{j k}\right)$ is the weak derivative of $a_{j k}$. Another consequence of the (regularity and ellipticity) assumptions imposed on $\sigma$ is the fact that the transition density $p_{\varepsilon}$ is smooth for $t>t_{0}$ and satisfies the forward Kolmogorov equation

$$
\frac{\partial}{\partial t} p_{\varepsilon}=\mathcal{L}^{*} p_{\varepsilon}=-\sum_{k} D_{k}\left(\tilde{b}_{k} p_{\epsilon}\right)+\frac{1}{2} \sum_{j, k} D_{k}\left(a_{j k} D_{k} p_{\epsilon}\right) .
$$

Furthermore, since $\psi$ does not depend on $t$, we have $p_{\varepsilon} \frac{\partial}{\partial t} \eta+\eta \frac{\partial}{\partial t} p_{\varepsilon}=0$, and so,

$$
\iint p_{\varepsilon} v^{i} \frac{\partial}{\partial t} \eta=-\iint \eta v^{i} \frac{\partial}{\partial t} p_{\varepsilon}=-\iint \eta v^{i} \mathcal{L}^{*} p_{\varepsilon} .
$$

Using the divergence form of $\mathcal{L}^{*}$ in (4.23) and the fact that $\eta(t, \cdot)$ is supported in $\beta(4)$, we conclude that

$$
-\int_{\beta(4)} \eta v^{i} \mathcal{L}^{*} p_{\varepsilon}=-\int_{\beta(4)} D\left(\eta v^{i}\right) \tilde{b} p_{\epsilon}+\frac{1}{2} \int_{\beta(4)}\left\langle D\left(\eta v^{i}\right), D p_{\varepsilon}\right\rangle_{a} .
$$

Similarly,

$$
\int_{\beta(4)} p_{\varepsilon} v^{i} \mathcal{L} \eta=\int_{\beta(4)} D \eta \tilde{b} v^{i} p_{\epsilon}-\frac{1}{2} \int_{\beta(4)}\left\langle D\left(p_{\varepsilon} v^{i}\right), D \eta\right\rangle_{a} .
$$


Finally, we integrate both (4.25) and (4.26) over $t \in\left[t_{1}, t_{2}\right]$, and combine them with (4.24), to conclude that

$$
\begin{aligned}
\iint\left(p_{\varepsilon} v^{i}\left(\frac{\partial}{\partial t} \eta+\mathcal{L} \eta\right)+p_{\varepsilon}\left\langle D v^{i}, D \eta\right\rangle_{a}\right) \\
=\iint\left(\frac{1}{2}\left\langle D\left(\eta v^{i}\right), D p_{\varepsilon}\right\rangle_{a}-\frac{1}{2}\left\langle D\left(p_{\varepsilon} v^{i}\right), D \eta\right\rangle_{a}\right. \\
\left.\quad+p_{\varepsilon}\left\langle D v^{i}, D \eta\right\rangle_{a}-D\left(\eta v^{i}\right) \tilde{b} p_{\epsilon}+D \eta \tilde{b} v^{i} p_{\epsilon}\right) \\
=\iint\left(\frac{1}{2}\left\langle D v^{i}, D \psi\right\rangle_{a}-\psi D v^{i} \tilde{b}\right) .
\end{aligned}
$$

Next, we multiply both sides of (4.22) by $-L$ and use (4.27) together with the uniform ellipticity of $\sigma$ and the fact that $\left|f^{i}\right| \leq C\left(|D v|^{2}+k\right)$ to obtain

$$
L^{2} \leq C\left(|L| \iint|\psi||D v|^{2}+|L| \iint|\psi| k+\iint(|L D \psi|+|L \psi \tilde{b}|)\left|D v^{i}\right|\right) .
$$

Hölder's inequality, applied to the third term on the right-hand side above, and use the fact that $|L| \leq C\|v\|_{\mathbb{L}^{\infty}} R^{d}$ for the first term yield

$$
\begin{aligned}
L^{2} \leq & C R^{d}\left(\iint \mathbf{1}_{\psi>0}|D \boldsymbol{v}|^{2}+\iint \mathbf{1}_{\psi>0} k\right)+\frac{1}{2} L^{2} \\
& +\frac{1}{2} \iint(|D \psi|+\psi|\tilde{b}|)^{2} \iint \mathbf{1}_{\psi>0}\left|D v^{i}\right|^{2} .
\end{aligned}
$$

To complete the proof, we use the first inequality in (4.2), apply Hölder's inequality to the integral $\iint \mathbf{1}_{\psi>0} k$, and use the boundedness of $\tilde{b}$ and $R \leq 1 / 4$ to obtain $\iint \psi^{2}|\tilde{b}|^{2} \leq_{C} R^{d}$ and

$$
\int|D \psi|^{2}=R^{d-2} \int|D \tilde{\psi}|
$$

so that

$$
\iint|D \psi|^{2} \leq\left(\theta_{2}-\theta_{1}\right) R^{d} \int|D \tilde{\psi}|^{2} \leq_{C} R^{d}
$$

Coming back to (4.20), we will estimate different terms on the right-hand side using Lemmas 4.9 and 4.11, together with a specific choice of $\boldsymbol{c}$.

LEMMA 4.12. There exists universal constant $C$ such that, for all $\varepsilon \in$ $\left(0, \varepsilon_{0} / 2\right]$ we have

$$
\begin{aligned}
& \sup _{\gamma(4 ; 4) \backslash \gamma(1,1)} R^{d} \mid \overline{\boldsymbol{v}(t, \cdot)} \psi \\
& \quad \leq\left. c\right|^{2} p_{\varepsilon} \\
& \quad \leq C \int_{\gamma(4 ; 4) \backslash \gamma(4 ; 1 / 2)}|D \boldsymbol{v}|^{2}\left(\frac{p_{\varepsilon}}{\delta_{\underline{\sigma} / 4}\left(\varepsilon+\varepsilon_{0} / 2\right)}+\frac{\delta_{\bar{\sigma}}\left(\varepsilon+\varepsilon_{0} / 2\right)}{R^{d}}\right)+R^{2-\frac{2+d}{q}}, \\
& \text { where } \boldsymbol{c}=\frac{\boldsymbol{v}\left(t_{0}+\left(\frac{1}{2} \varepsilon_{0} \wedge \tilde{T}\right) R^{2}, \cdot\right)}{\psi} \psi
\end{aligned}
$$


Proof. We fix $(t, x) \in \gamma(4 ; 4) \backslash \gamma(1,1)$, set $\tilde{t}=\left(t-t_{0}\right) / R^{2}$ and define $\theta_{1}=$ $\min \left(\tilde{t},\left(\varepsilon_{0} / 2\right) \wedge \tilde{T}\right)$, and $\theta_{2}=\max \left(\tilde{t},\left(\varepsilon_{0} / 2\right) \wedge \tilde{T}\right)$, so that $0 \leq \theta_{1} \leq \theta_{2} \leq 4$, and Lemma 4.11 can be applied. We distinguish the following two cases:

Case 1: $\varepsilon_{0} / 2 \leq \tilde{t}$. In this case, $\theta_{1}=\epsilon_{0} / 2$ and the estimate (4.15) of Lemma 4.5 applies. Lemma 4.11 , with (4.14) applied to the $p_{\epsilon}$ outside the integral in the second inequality, yields

$$
\begin{aligned}
& R^{d}\left|\overline{\boldsymbol{v}(t, \cdot)}^{\psi}-\boldsymbol{c}\right|^{2} p_{\varepsilon} \leq C p_{\varepsilon} \iint_{\gamma\left(\theta_{2} ; 4\right) \backslash\left(\gamma\left(\theta_{1} ; 4\right) \cup \gamma(4 ; 1 / 2)\right)}|D \boldsymbol{v}|^{2} \frac{R^{d} p_{\varepsilon}}{\delta_{\underline{\sigma} / 4}\left(\varepsilon+\varepsilon_{0} / 2\right)} \\
& +p_{\varepsilon} R^{2+d-\frac{2+d}{q}} \\
& \leq_{C} R^{d} p_{\varepsilon} \frac{1}{\delta_{\underline{\sigma} / 4}\left(\varepsilon+\varepsilon_{0} / 2\right)} \iint_{\gamma(4,4) \backslash \gamma(4,1 / 2)}|D v|^{2} p_{\varepsilon} \\
& +p_{\epsilon} R^{2+d-\frac{2+d}{q}} \\
& \leq_{C} \frac{1}{\delta_{\underline{\sigma} / 4}\left(\varepsilon+\varepsilon_{0} / 2\right)} \iint_{\gamma(4,4) \backslash \gamma(4,1 / 2)}|D \boldsymbol{v}|^{2} p_{\varepsilon}+R^{2-\frac{2+d}{q}} .
\end{aligned}
$$

Case 2: $\varepsilon_{0} / 2>\tilde{t}$. Since $\varepsilon_{0} \leq 1$, we have $\tilde{t} \leq 1 / 2$. Therefore, $(t, x) \in \gamma(1 / 2,4) \backslash$ $\gamma(1 / 2,1)$, and so $x \notin \beta(1)$. Now that its conditions are met, inequality (4.16) of Lemma 4.5 , together with the fact that $\delta_{\bar{\sigma}}$ is bounded from above and Lemma 4.11, implies that

$$
R^{d}\left|\overline{\boldsymbol{v}(t, \cdot)}^{\psi}-\boldsymbol{c}\right| p_{\varepsilon} \leq_{C} \delta_{\bar{\sigma}}\left(\varepsilon+\varepsilon_{0} / 2\right) R^{-d} \iint_{\gamma(4,4) \backslash \gamma(4,1 / 2)}|D \boldsymbol{v}|^{2}+R^{2-\frac{2+d}{q}} .
$$

LEMMA 4.13. There exists universal constant $C$ such that, for all $\varepsilon \in$ $\left(0, \varepsilon_{0} / 2\right]$, and all $t \in\left[t_{0}, t_{0}+4 R^{2}\right]$, we have

$\int_{D}\left|\boldsymbol{v}(t, \cdot)-\overline{\boldsymbol{v}(t, \cdot)}{ }^{\psi}\right|^{2} p_{\varepsilon} \leq_{C} R^{2} \int_{D^{\prime}}|D \boldsymbol{v}(t, \cdot)|^{2}\left(\frac{p_{\varepsilon}}{\delta_{\underline{\sigma} / 4}\left(\varepsilon+\varepsilon_{0} / 2\right)}+\frac{\delta_{\bar{\sigma}}\left(\varepsilon+\varepsilon_{0} / 2\right)}{R^{d}}\right)$, where either $\left(D, D^{\prime}\right)=(\beta(2) \backslash \beta(1), \beta(4) \backslash \beta(1 / 2))$, or $\left(D, D^{\prime}\right)=(\beta(2), \beta(4))$.

PROOF. We omit the details, since the same strategy as in the proof of Lemma 4.12, namely separating the cases $\tilde{t} \leq \varepsilon_{0} / 2$ and $\tilde{t} \geq \varepsilon_{0} / 2$, where $\tilde{t}=$ $\left(t-t_{0}\right) / R^{2}$, and using the corresponding estimates from Lemma 4.5 , but this time together with Lemma 4.9, can be applied.

LEMMA 4.14. Set $\alpha^{\prime}=\min \left(\alpha, 1-\frac{1}{q}\left(1+\frac{d}{2}\right)\right)>0$. There exists a universal constant $C$ such that, for each $\varepsilon \in\left(0, \varepsilon_{0} / 2\right]$ we have

$$
\begin{aligned}
& \iint_{\gamma(1 ; 1 / 2)}|D \boldsymbol{v}|^{2} p_{\varepsilon} \\
& \quad \leq R^{2 \alpha^{\prime}}+\iint_{\gamma(4,4) \backslash \gamma(1,1 / 2)}|D v|^{2}\left(\frac{p_{\varepsilon}}{\delta_{\underline{\sigma} / 4}\left(\varepsilon+\varepsilon_{0} / 2\right)}+\frac{\delta_{\bar{\sigma}}\left(\varepsilon+\varepsilon_{0} / 2\right)}{R^{d}}\right) .
\end{aligned}
$$


PROOF. With $c={\overline{\left.v\left(t_{0}+\frac{1}{2} \varepsilon_{0} \wedge \tilde{T}\right) R^{2}, \cdot\right)}}^{\psi}$, as in Lemma 4.12, we start from the inequality:

$$
|\boldsymbol{v}(t, x)-\boldsymbol{c}|^{2} p_{\varepsilon} \leq 2\left|\boldsymbol{v}(t, x)-\overline{\boldsymbol{v}(t, \cdot)}{ }^{\psi}\right|^{2} p_{\varepsilon}+2|\overline{\boldsymbol{v}(t, \cdot)} \psi+\boldsymbol{c}|^{2} p_{\varepsilon} .
$$

With $\tilde{t}=\left(t-t_{0}\right) / R^{2}$, we integrate (4.29) over $D(t)=\beta(2) \backslash \beta(1)$ when $\tilde{t} \in[0,1]$ and over $D(t)=\beta(2)$ when $\tilde{t} \in(1,4]$. Thanks to Lemmas 4.12 and 4.13 we get, for $t \in\left[t_{0}, t_{0}+4 R^{2}\right]$,

$$
\begin{aligned}
& R^{-2} \int_{D(t)}|\boldsymbol{v}(t, \cdot)-\boldsymbol{c}|^{2} p_{\varepsilon} \\
& \quad \leq C \int_{D^{\prime}(t)}|D \boldsymbol{v}(t, \cdot)|^{2}\left(\frac{p_{\varepsilon}(t, \cdot)}{\delta_{\underline{\sigma} / 4}\left(\varepsilon+\varepsilon_{0} / 2\right)}+\frac{\delta_{\bar{\sigma}}\left(\varepsilon+\varepsilon_{0} / 2\right)}{R^{d}}\right) \\
& \quad+R^{-2} \iint_{\gamma(4 ; 4) \backslash \gamma(1 ; 1 / 2)}|D \boldsymbol{v}|^{2}\left(\frac{p_{\varepsilon}}{\delta_{\underline{\sigma} / 4}\left(\varepsilon+\varepsilon_{0} / 2\right)}+\frac{\delta_{\bar{\sigma}}\left(\varepsilon+\varepsilon_{0} / 2\right)}{R^{d}}\right) \\
& \quad+R^{-\frac{2+d}{q}}
\end{aligned}
$$

where $D^{\prime}(t)=\beta(4) \backslash \beta(1 / 2)$ for $\tilde{t} \in[0,1]$ and $D^{\prime}(t)=B(4)$, for $\tilde{t} \in(1,4]$. Then we integrate (4.30) over $t \in\left[t_{0}, t_{0}+4 R^{2}\right]$ to obtain

$$
\begin{aligned}
& R^{-2} \iint_{\gamma(4,2) \backslash \gamma(1,1)}|\boldsymbol{v}-\boldsymbol{c}|^{2} p_{\varepsilon} \\
& \leq C \iint_{\gamma(4 ; 4) \backslash \gamma(1 ; 1 / 2)}|D \boldsymbol{v}|^{2}\left(\frac{p_{\varepsilon}}{\delta_{\underline{\sigma} / 4}\left(\varepsilon+\varepsilon_{0} / 2\right)}+\frac{\delta_{\bar{\sigma}}\left(\varepsilon+\varepsilon_{0} / 2\right)}{R^{d}}\right) \\
& \quad+R^{2-\frac{2+d}{q}} .
\end{aligned}
$$

Consider, now, the case when $\tilde{T} \leq 4$, that, $t_{0} \geq T-4 R^{2}$. Since $g$ is $\alpha$-Hölder and supp $\psi \subseteq \beta(4)$, we have $\sup _{\beta(4)}\left|\boldsymbol{g}-\overline{\boldsymbol{g}}^{\psi}\right| \leq \max _{x, x^{\prime} \in B(4)}\left|\boldsymbol{g}(x)-\boldsymbol{g}\left(x^{\prime}\right)\right| \leq_{C}$ $R^{\alpha}$. This inequality and Lemma 4.11 combined, together with $R \leq 1 / 4$ together with (4.15) in Lemma 4.5 applied to the last inequality, imply that

$$
\begin{aligned}
\int_{\beta(2)}|\boldsymbol{g}-\boldsymbol{c}|^{2} p_{\varepsilon}(T, \cdot) \leq & \int_{\beta(2)}\left|\boldsymbol{g}-\overline{\boldsymbol{g}}^{\psi}\right|^{2} p_{\epsilon}(T, \cdot)+2\left|\overline{\boldsymbol{g}}^{\psi}-\boldsymbol{c}\right|^{2} \\
\leq & R^{2 \alpha}+\left|\overline{\boldsymbol{v}(T, \cdot)} \psi-\overline{\boldsymbol{v}\left(t_{0}+\varepsilon_{0} R^{2} / 2, \cdot\right)} \psi\right|^{2} \\
\leq & R^{2 \alpha}+R^{-d} \iint_{\left[t_{0}+\varepsilon_{0} R^{2} / 2, T\right] \times(B(4) \backslash B(1 / 2))}|D \boldsymbol{v}|^{2} \\
& +R^{2-\frac{d+2}{q}} \\
\leq C & R^{2 \alpha^{\prime}}+\frac{1}{\delta_{\underline{\sigma} / 4}\left(\varepsilon+\varepsilon_{0} / 2\right)} \iint_{\gamma(4,4) \backslash \gamma(4,1 / 2)}|D \boldsymbol{v}|^{2} p_{\varepsilon} .
\end{aligned}
$$


Finally, we combine the estimates (4.31) and (4.32) with (4.20) (shrinking and extending the domains of integration appropriately) and use $R \leq 1 / 4$ to obtain (4.28).

4.5. Hole-filling. The following technique is so called "hole-filling," which was first applied to parabolic systems by [53]. In the previous subsections, $\left(t_{0}, x_{0}\right)$ and $R$ are fixed. Now they will be varied in $[0, T] \times B_{n-1}$ and $(0,1 / 4]$. However, the center of the ball $B_{n-1}$ is still fixed at $b_{0}$. It is important to note that none of constants $C$ below depends on $\left(t_{0}, x_{0}\right)$ and $R$.

LEMMA 4.15. There exists an universal constant $C$ such that

$$
\begin{aligned}
& \sup _{\varepsilon \in\left(0, \varepsilon_{0} / 4\right]} \iint_{\gamma(1 / 4 ; 1 / 2)}|D \boldsymbol{v}|^{2} p_{\varepsilon} \\
& \quad \leq \kappa\left(\varepsilon_{0}\right) \sup _{\varepsilon^{\prime} \in\left(0,16 \varepsilon_{0}\right]} \iint_{\gamma(16 ; 4)}|D \boldsymbol{v}|^{2} p_{\varepsilon^{\prime}}+C R^{2 \alpha^{\prime}},
\end{aligned}
$$

where $\alpha^{\prime}=\min \left(\alpha, 1-\frac{1}{q}\left(1+\frac{d}{2}\right)\right)>0$, and $\kappa(\varepsilon)=\left(1+\frac{1}{2} \delta_{\underline{\sigma} / 4}(\varepsilon)\right) /\left(1+\delta_{\underline{\sigma} / 4}(\varepsilon)\right)$.

PROOF. Inequality (4.15) of Lemma 4.5 yields $p_{\varepsilon_{0}} \geq_{C} R^{-d}$ on $\gamma(4 ; 4)$. Given $\varepsilon \in\left(0, \varepsilon_{0} / 2\right]$, this inequality, combined with (4.28), yields

$$
\begin{aligned}
& \iint_{\gamma(1 ; 1 / 2)}|D \boldsymbol{v}|^{2} p_{\varepsilon} \leq_{C} R^{2 \alpha^{\prime}}+\frac{1}{\delta_{\underline{\sigma} / 4}\left(\varepsilon+\varepsilon_{0} / 2\right)} \iint_{\gamma(4,4) \backslash \gamma(1,1 / 2)}|D \boldsymbol{v}|^{2} p_{\varepsilon} \\
& +\delta_{\bar{\sigma}}\left(\varepsilon+\varepsilon_{0} / 2\right) \iint_{\gamma(4 ; 4)}|D v|^{2} p_{\varepsilon_{0}} \text {. }
\end{aligned}
$$

Let $C_{0}$ denote the constant $C$ from (4.34); we assume, without loss of generality, that $C_{0} \geq 1$. Adding $\frac{C_{0}}{\delta_{\sigma / 4}\left(\varepsilon+\varepsilon_{0} / 2\right)} \iint_{\gamma(1 ; 1 / 2)}|D \boldsymbol{v}|^{2} p_{\varepsilon}$ to both sides of (4.34) and dividing throughout by $1+\frac{C_{0}}{\delta_{\underline{\sigma} / 4}\left(\varepsilon+\varepsilon_{0} / 2\right)}$ yields

$$
\begin{aligned}
& \iint_{\gamma(1 ; 1 / 2)}|D \boldsymbol{v}|^{2} p_{\varepsilon} \\
& \quad \leq C_{0} R^{2 \alpha^{\prime}}+\kappa^{\prime}\left(\varepsilon+\varepsilon_{0} / 2\right)\left(\iint_{\gamma(4,4)}|D \boldsymbol{v}|^{2} p_{\varepsilon}+\iint_{\gamma(4 ; 4)}|D \boldsymbol{v}|^{2} p_{\varepsilon_{0}}\right),
\end{aligned}
$$

where $\kappa^{\prime}(\varepsilon)=\left(1+\delta_{\underline{\sigma} / 4} \delta_{\bar{\sigma}}\right) /\left(C_{0}+\delta_{\underline{\sigma} / 4}\right)$. Our choice of the constant $\varepsilon_{0}$ implies that $\kappa^{\prime}(\varepsilon) \leq \kappa(\varepsilon)$, for $\varepsilon \leq \varepsilon_{0}$. Moreover, $\kappa$ is strictly decreasing on $\left[0, \varepsilon_{0}\right]$, so $\kappa\left(\varepsilon_{0} / 2\right) \geq$ $\kappa\left(\varepsilon+\varepsilon_{0} / 2\right)$, for $\varepsilon \in\left(0, \varepsilon_{0} / 2\right]$. Therefore, extending domains on the right-hand side and shrink domains on the left-hand side, we obtain

$$
\begin{aligned}
\iint_{\gamma(1 / 4 ; 1 / 2)}|D \boldsymbol{v}|^{2} p_{\varepsilon} & \leq \iint_{\gamma(1 ; 1 / 2)}|D \boldsymbol{v}|^{2} p_{\varepsilon} \\
& \leq C_{0} R^{2 \alpha^{\prime}}+\kappa\left(\varepsilon_{0} / 2\right) \sup _{\varepsilon^{\prime} \in\left(0,16 \varepsilon_{0}\right]} \iint_{\gamma(16 ; 4)}|D \boldsymbol{v}|^{2} p_{\varepsilon^{\prime}} .
\end{aligned}
$$

Maximizing over $\varepsilon \in\left(0, \varepsilon_{0} / 4\right]$ on the left-hand side completes the argument. 
Proposition 4.16. There exists universal constants $C, \alpha_{0}>0$ such that

$$
\sup _{R \in(0,1 / 4]} R^{-d-2 \alpha_{0}} \iint_{\gamma(1 ; 1)}|D \boldsymbol{v}|^{2} \leq C .
$$

PROOF. In this proof, we need to vary $R$ and do not consider it fixed, while we still keep $\left(t_{0}, x_{0}\right)$ fixed. Hence, we include explicit dependence on $R$ in the notation as in, for example, $\gamma_{R}(1,1)$. Given $\alpha_{0} \in\left(0, \alpha^{\prime}\right]$, with $\alpha^{\prime}$ as in Lemma 4.15, we define

$$
\lambda_{\alpha_{0}}(R)=\sup _{\varepsilon \in\left(0, \varepsilon_{0}\right]} R^{-2 \alpha_{0}} \iint_{\gamma_{R}(1,1)}|D \boldsymbol{v}|^{2} p\left(t_{0}-\varepsilon R^{2}, x_{0}, \cdot, \cdot\right) .
$$

Lemma 4.15 implies that there exists a universal constant $C_{0}>0$ such that

$$
\lambda_{\alpha_{0}}\left(\frac{1}{2} R\right) \leq C_{0}+v\left(\alpha_{0}\right) \lambda_{\alpha_{0}}(4 R) \quad \text { for } R \in(0,1 / 4],
$$

where, with $\kappa<1$ as in Lemma 4.15, we have $v\left(\alpha_{0}\right)=8^{2 \alpha_{0}} \kappa\left(\varepsilon_{0}\right)$. Choosing $0<$ $\alpha_{0} \leq \alpha^{\prime}$ small enough so that $v_{0}=v\left(\alpha_{0}\right)<1$, we obtain

$$
\lambda_{\alpha_{0}}\left(\frac{1}{2} R\right) \leq C_{0}+v_{0} \lambda_{\alpha_{0}}(4 R) \quad \text { for } R \in(0,1 / 4] .
$$

On the other hand, Proposition 4.7 together with the boundedness of $v$ imply that $\lambda_{\alpha_{0}}(\cdot)$ is bounded on compact segments of $(0, \infty)$. This and (4.37) combined yield

$$
\sup _{R \leq 1 / 4} \lambda_{\alpha_{0}}(R) \leq C_{1},
$$

for some universal constant $C_{1}$. The statement then follows from specializing the supremum in the definition of $\varphi$ to $\varepsilon=\varepsilon_{0}$ and estimating $p_{\varepsilon_{0}}$ using (4.14) of Lemma 4.5.

The following result completes the proof of Theorem 2.5.

COROLlary 4.17 (Uniform $C^{\alpha}$-bounds). There exist universal constants $C$ and $\alpha_{0}>0$ such that

$$
[\boldsymbol{v}]_{\alpha_{0} ; B_{n-1}} \leq C .
$$

Proof. Keeping $\left(t_{0}, x_{0}\right) \in[0, T] \times B_{n-1}$ and $R \leq 1 / 4$ fixed, we set

$$
\boldsymbol{c}=\frac{1}{R^{2}} \int_{t_{0}}^{t_{0}+R^{2}} \overline{\boldsymbol{v}(t, \cdot)} \psi d t
$$

so that

$$
\int_{\beta(1)}|\boldsymbol{v}(t, \cdot)-\boldsymbol{c}|^{2} \leq_{C} \int_{\beta(1)}\left|\boldsymbol{v}(t, \cdot)-\overline{\boldsymbol{v}(t, \cdot)}{ }^{\psi}\right|^{2}+R^{d}\left|\overline{\boldsymbol{v}(t, \cdot)}{ }^{\psi}-\boldsymbol{c}\right|^{2} .
$$


Applying Lemmas 4.9 and 4.11 to the two terms on the right-hand side respectively, we obtain

$$
\begin{aligned}
& \left.\int_{\beta(1)}|\boldsymbol{v}(t, \cdot)-\overline{\boldsymbol{v}(t, \cdot)} \psi|\right|^{2} \leq_{C} R^{2} \int_{\beta(4)}|D \boldsymbol{v}(t, \cdot)|^{2} \quad \text { and } \\
& R^{d}\left|\overline{\boldsymbol{v}(t, \cdot)}{ }^{\psi}-\boldsymbol{c}\right|^{2} \leq_{C} \iint_{\gamma(1 ; 4)}|D \boldsymbol{v}|^{2}+R^{d+2-\frac{2+d}{q}},
\end{aligned}
$$

so that, an integration of (4.39) over $\left[t_{0}, t_{0}+R^{2}\right]$ yields

$$
\iint_{\gamma(1 ; 1)}|\boldsymbol{v}-\boldsymbol{c}|^{2} \leq_{C} R^{2} \iint_{\gamma(1 ; 4)}|D \boldsymbol{v}|^{2}+R^{2+d+\left(2-\frac{2+d}{q}\right)} .
$$

Dividing both sides by $R^{d+2+2 \alpha_{0}}$, where $\alpha_{0}$ is from Proposition 4.16, and using the same proposition on the right-hand side, we obtain a universal constant $C$ such that $\iint_{\gamma(1 ; 1)}|\boldsymbol{v}-\boldsymbol{c}|^{2} \leq_{C} R^{d+2+2 \alpha_{0}}$ for all $R \leq 1 / 4$. Finally, $\boldsymbol{c}=\overline{\boldsymbol{v}}_{\gamma(1 ; 1)}$ minimizes the integral $\iint_{\gamma(1 ; 1)}|\boldsymbol{v}-\boldsymbol{c}|^{2}$, and thus, we have

$$
\sup _{R \in(0,1 / 4]} R^{-d-2-2 \alpha_{0}} \iint_{\gamma(1 ; 1)}\left|\boldsymbol{v}-\overline{\boldsymbol{v}}_{\gamma(1 ; 1)}\right|^{2} \leq C .
$$

The constant $C$ of (4.40) above does not depend on $\left(t_{0}, x_{0}\right) \in[0, T] \times B_{n-1}$, so $\boldsymbol{v}$ belongs to the ball of radius $\sqrt{C}$ in the Campanato space $\hat{C}^{\alpha_{0}}\left([0, T] \times B_{n-1}\right)$, where

$$
\begin{aligned}
\hat{C}^{\alpha_{0}}\left([0, T] \times B_{n-1}\right) & \\
:= & \left\{\boldsymbol{v} \in \mathbb{L}^{2}: \sup _{\left(t_{0}, x_{0}\right) \in[0, T] \times B_{n-1}, R \in(0,1 / 4]} R^{-d-2-2 \alpha_{0}}\right. \\
& \left.\times \iint_{\gamma_{t_{0}, x_{0}, R}(1 ; 1)}\left|\boldsymbol{v}-\overline{\boldsymbol{v}}_{\gamma_{t_{0}, x_{0}, R}(1 ; 1)}\right|^{2}<\infty\right\} .
\end{aligned}
$$

The (topological) equivalence of the Campanato space $\hat{C}^{\alpha_{0}}\left([0, T] \times B_{n-1}\right)$ with the natural metric, and the Hölder spaces $C^{\alpha_{0}}\left([0, T] \times B_{n-1}\right)$ (see, e.g., [47], Chapter IV, Section 2, page 49) implies that $[\boldsymbol{v}]_{\alpha_{0} ; B_{n-1}}$ admits a universal bound.

\section{Additional proofs.}

5.1. Proof of Theorem 2.8. Thanks to our notational convention at the beginning of Section 4 , the index $m$ was suppressed in the statement of Corollary 4.17. The dependence on $k_{n}^{m}$ is through its $\mathbb{L}^{q}$-norm on $[0, T] \times B_{n}\left(b_{0}\right)$, which is assumed to be bounded uniformly in $m$. With the conditions (1)-(4) of Theorem 2.5 holding uniformly in $m$, we have a universal constant $C$ such that $\left[\boldsymbol{v}_{m}\right]_{\alpha_{0} ; B_{n}\left(b_{0}\right)} \leq C$, for all $m$. Combining this uniform Hölder estimate and the uniform bound in condition (2) of Theorem 2.5, we apply the Arzelá-Ascoli theorem 
on $[0, T] \times B_{n}\left(b_{0}\right)$ to extract a subsequence of $\left\{\boldsymbol{v}^{m}\right\}$, which converges uniformly. A diagonal procedure then produces another subsequence-still denoted by $\left\{\boldsymbol{v}^{m}\right\}$, as well as a continuous function $\boldsymbol{v}:[0, T] \times \mathbb{R}^{d} \rightarrow \mathbb{R}^{N}$ such that $\boldsymbol{v}^{m} \rightarrow \boldsymbol{v}$, locally uniformly. Thanks to the preservation of Hölder continuity under uniform convergence, the function $\boldsymbol{v}$ belongs to the local Hölder space $C_{\mathrm{loc}, b_{0}}^{\left\{\alpha_{n}\right\}}$, for some sequence $\left\{\alpha_{n}^{\prime}\right\}$ in $(0,1]$.

Having picked and fixed $n \in \mathbb{N}$ and the initial condition $(t, x) \in[0, T] \times \mathbb{R}^{d}$, we set $X=X^{t, x}$ and define the exit time

$$
\tau_{n}=\inf \left\{u \geq t: X_{u} \notin B_{n}\left(b_{0}\right)\right\},
$$

as well as the following two sequences of processes:

$$
\boldsymbol{Y}_{u}^{(m)}=\boldsymbol{v}^{m}\left(u, X_{u}^{\tau_{n}}\right) \quad \text { and } \quad \boldsymbol{Z}_{u}^{(m)}=\boldsymbol{w}^{m}\left(u, X_{u}\right) \mathbf{1}_{\left\{u<\tau_{n}\right\}}, \quad u \in[t, T] .
$$

Since $\left(\boldsymbol{v}^{m}, \boldsymbol{w}^{m}\right)$ is a Markovian solution to the system (2.5), the process $\boldsymbol{Y}^{m}$ is a semimartingale whose finite-variation part is given by

$$
-\int_{t} \boldsymbol{f}^{m}\left(u, X_{u}^{\tau_{n}}, \boldsymbol{Y}_{u}^{(m)}, \boldsymbol{Z}_{u}^{(m)}\right) \mathbf{1}_{\left\{u \leq \tau_{n}\right\}} d u .
$$

Condition (3) in Theorem 2.5 and Lemma 4.3 imply that these, finite-variation, parts admit a uniform bound in total variation, that is,

$$
\begin{aligned}
\mathbb{E}^{t, x} & \int_{t}^{T}\left[\left|\boldsymbol{f}^{m}\left(u, X_{u}^{\tau_{n}}, \boldsymbol{Y}_{u}^{(m)}, \boldsymbol{Z}_{u}^{(m)}\right)\right| \mathbf{1}_{\left\{u \leq \tau_{n}\right\}} d u\right] \\
& \leq C(n) \mathbb{E}^{t, x}\left[\int_{t}^{T}\left(\left|\boldsymbol{Z}_{u}^{(m)}\right|^{2}+k_{n}\left(u, X_{u}\right)\right) \mathbf{1}_{\left\{X_{u} \in B_{n}\left(b_{0}\right)\right\}} d u\right] \leq C(n) .
\end{aligned}
$$

Moreover, the uniform convergence of $\boldsymbol{v}^{m}$ on $B_{n}\left(b_{0}\right)$ implies that the convergence $\boldsymbol{Y}^{(m)} \rightarrow \boldsymbol{Y}^{n}=\boldsymbol{v}\left(\cdot, X^{\tau_{n}}\right)$ is also uniform.

Uniform ellipticity of $\sigma$ and Itô's formula applied to $\left|\boldsymbol{Y}^{(m)}-\boldsymbol{Y}^{\left(m^{\prime}\right)}\right|^{2}$ yield

$$
\begin{aligned}
\mathbb{E}^{t, x}\left[\int_{t}^{T}\left|\boldsymbol{Z}_{u}^{(m)}-\boldsymbol{Z}_{u}^{\left(m^{\prime}\right)}\right|^{2} d u\right] \\
\quad \leq C\left\|\boldsymbol{Y}_{\tau_{n}}^{(m)}-\boldsymbol{Y}_{\tau_{n}}^{\left(m^{\prime}\right)}\right\|_{\mathbb{L}^{\infty}}^{2} \\
\quad+\left\|\sup _{u \in\left[0, \tau_{n}\right]}\left|\boldsymbol{Y}_{u}^{(m)}-\boldsymbol{Y}_{u}^{\left(m^{\prime}\right)}\right|\right\|_{\mathbb{L}^{\infty}} \mathbb{E}^{t, x}\left[\int_{t}^{T}\left|\boldsymbol{f}_{u}^{m}\right|+\left|\boldsymbol{f}_{u}^{m^{\prime}}\right| d u\right],
\end{aligned}
$$

where $\boldsymbol{f}_{u}^{m}=\boldsymbol{f}^{m}\left(u, X_{u}^{\tau_{n}}, \boldsymbol{Y}_{u}^{(m)}, \boldsymbol{Z}_{u}^{(m)}\right) \mathbf{1}_{\left\{u \leq \tau_{n}\right\}}$ for all $m, m^{\prime} \in \mathbb{N}$. The uniform bound in (5.1) implies now that the sequence $\left\{\boldsymbol{Z}^{(m)}\right\}$ is Cauchy in $\mathbb{L}^{2}$ uniformly for $(t, x) \in[0, T] \times B_{n}\left(b_{0}\right)$, with limit $\boldsymbol{Z}^{n}$. A subsequence, still labeled $\left\{\boldsymbol{Z}^{(m)}\right\}$, converges Leb $\otimes \mathbb{P}$-a.e. toward the same limit,

$$
\boldsymbol{w}^{m}\left(u, X_{u}\right) \mathbf{1}_{\left\{u \leq \tau_{n}\right\}} \rightarrow \boldsymbol{Z}_{u}^{n},
$$


uniformly for $(t, x) \in[0, T] \times B_{n}\left(b_{0}\right)$. So it follows that

$$
\boldsymbol{Z}_{u}^{n}=\boldsymbol{Z}_{u} \mathbf{1}_{\left\{u \leq \tau_{n}\right\}}, \quad \text { Leb } \otimes \mathbb{P} \text {-a.s. }
$$

where

$$
\boldsymbol{Z}_{u}=\boldsymbol{w}\left(u, X_{u}\right) \quad \text { and } \quad \boldsymbol{w}\left(t^{\prime}, x^{\prime}\right)=\liminf _{m} \boldsymbol{w}^{m}\left(t^{\prime}, x^{\prime}\right) \quad \text { componentwise. }
$$

Therefore, by Itô's isometry, for almost all $t^{\prime} \geq t$ we have

$$
\int_{t}^{\tau_{n} \wedge t^{\prime}} Z_{u}^{(m)} \sigma\left(u, X_{u}\right) d W_{u} \rightarrow \int_{t}^{\tau_{n} \wedge t^{\prime}} Z_{u} \sigma\left(u, X_{u}\right) d W_{u}, \quad \text { a.s. }
$$

Next, we show that

$$
\int_{t}^{\tau_{n} \wedge t^{\prime}} \boldsymbol{f}^{m}\left(u, X_{u}, \boldsymbol{Y}_{u}^{(m)}, \boldsymbol{Z}_{u}^{(m)}\right) d u \rightarrow \int_{t}^{\tau_{n} \wedge t^{\prime}} \boldsymbol{f}\left(u, X_{u}, \boldsymbol{Y}_{u}, \boldsymbol{Z}_{u}\right) d u
$$

as $m \rightarrow \infty$, for almost all $t^{\prime} \geq t$. For that, we first observe that, thanks to the assumptions placed on the convergence $\boldsymbol{f}^{m} \rightarrow \boldsymbol{f}$, we have

$$
\boldsymbol{f}^{m}\left(u, X_{u}, \boldsymbol{Y}_{u}^{(m)}, \boldsymbol{Z}_{u}^{(m)}\right) \mathbf{1}_{\left\{u \leq \tau_{n}\right\}} \rightarrow \boldsymbol{f}\left(u, X_{u}, \boldsymbol{Y}_{u}, \boldsymbol{Z}_{u}\right) \mathbf{1}_{\left\{u \leq \tau_{n}\right\}}, \quad \lambda \otimes \mathbb{P} \text {-a.e. }
$$

This is, however, enough to ensure the $\lambda \otimes \mathbb{P}$-convergence, which in turn, implies (5.3). Indeed, we have

$$
\begin{gathered}
\left|\boldsymbol{f}^{m}\left(u, X_{u}, \boldsymbol{Y}_{u}^{(m)}, \boldsymbol{Z}_{u}^{(m)}\right)-\boldsymbol{f}\left(u, X_{u}, \boldsymbol{Y}_{u} \boldsymbol{Z}_{u}\right)\right| \mathbf{1}_{\left\{u \leq \tau_{n}\right\}} \\
\quad \leq_{C}\left(\left|\boldsymbol{Z}^{m}\right|^{2}+|\boldsymbol{Z}|^{2}+k_{n}\left(t, X_{u}\right)\right) \mathbf{1}_{\left\{u \leq \tau_{n}\right\}},
\end{gathered}
$$

with the right-hand side Leb $\otimes \mathbb{P}$-uniformly integrable, thanks to the $\mathbb{L}^{2}($ Leb $\otimes \mathbb{P})$ convergence of $\boldsymbol{Z}^{(m)}$.

It is straightforward now to let $n \rightarrow \infty$ and conclude that the pair $(\boldsymbol{v}, \boldsymbol{w})$ is a Markovian solution to (2.7). To show that $\boldsymbol{w}=D \boldsymbol{v}$ in the weak sense, we simply note that the proof of Lemma 4.4 applies verbatim.

5.2. Proof of Theorem 2.9. We start with a uniform bmo estimate, which will also be used in the proof of uniqueness. For a Borel function $w:[0, T] \times \mathbb{R}^{d} \rightarrow$ $\mathbb{R}^{N \times d}$ and a constant $\delta>0$, we define its bmo $(\delta)$-norm by

$$
\|\boldsymbol{w}\|_{\mathrm{bmo}(\delta)}^{2}=\sup _{t \in[\delta, T]} \sup _{t-\delta \leq \tau \leq t}\left\|\mathbb{E}_{\tau}\left[\int_{\tau}^{t}\left|\boldsymbol{w}\left(u, X_{u}\right)\right|^{2} d u\right]\right\|_{\mathbb{L}^{\infty}},
$$

where $\tau$ is any stopping time taking value in $[t-\delta, t]$. We say that $\boldsymbol{w} \in \mathrm{uBMO}$ loc if $\lim _{\delta \searrow 0}\|\boldsymbol{w}\|_{\mathrm{bmo}(\delta)}=0$; this, stronger, notion of bmo-regularity will play a role in the uniqueness proof below. We start with a well-known estimate whose proof we include for the reader's convenience. 
LEMMA 5.1. For any $t \in[\delta, T]$ and any stopping time $\tau$ taking value in $[t-\delta, t]$, and $\alpha \in(0,1]$, we have

$$
\mathbb{E}_{\tau}\left[\left|X_{t}-X_{\tau}\right|^{\alpha}\right] \leq C \delta^{\alpha / 2}
$$

where $C$ depends only on $\alpha, d,\|b\|_{\mathbb{L}^{\infty}}$ and $\|\sigma\|_{\mathbb{L}^{\infty}}$.

PROOF. Using the Burkholder-Davis-Gundy inequality, we obtain

$\mathbb{E}_{\tau}\left[\left|X_{t}-X_{\tau}\right|\right] \leq C \mathbb{E}_{\tau}\left[\left|\int_{\tau}^{t} b\left(u, X_{u}\right) d u\right|\right]+\mathbb{E}_{\tau}\left[\left(\int_{\tau}^{t}\left|\sigma\left(u, X_{u}\right)\right|^{2} d u\right)^{\frac{1}{2}}\right] \leq C \delta^{1 / 2}$.

The inequality (5.4) now follows from the fact that $\mathbb{E}_{\tau}\left[\left|X_{t}-X_{\tau}\right|^{\alpha}\right] \leq$ $\mathbb{E}_{\tau}\left[\left|X_{t}-X_{\tau}\right|\right]^{\alpha}$.

Proposition 5.2. Suppose that, for some $c>0$, there exists $(h, k) \in$ $\mathbf{L y}(\boldsymbol{f}, c)$ with $k \in \mathbb{L}^{\infty}$. Then $\boldsymbol{w} \in u B M O_{\mathrm{loc}}$ for any locally Hölderian solution $(\boldsymbol{v}, \boldsymbol{w})$ to (2.2) with $\boldsymbol{v} \in C_{\mathrm{loc}}^{\left\{\alpha_{n}\right\}}$ and $\|\boldsymbol{v}\|_{\mathbb{L}^{\infty}} \leq c$.

Proof. Given $(h, k) \in \mathbf{L y}(\boldsymbol{f}, c)$ and $t-\delta \leq \tau \leq t$, we apply Itô's formula to $h\left(\boldsymbol{Y}_{u}\right)$, where $\boldsymbol{Y}_{u}=\boldsymbol{v}\left(u, X_{u}\right)$. With the boundedness of $\boldsymbol{v}$ and a localization argument guaranteeing that the expectations of the local-martingale parts vanish, we obtain

$$
\mathbb{E}_{\tau}\left[h\left(\boldsymbol{v}\left(t, X_{t}\right)\right)-h\left(\boldsymbol{v}\left(\tau, X_{\tau}\right)\right)\right] \geq \mathbb{E}_{\tau}\left[\int_{\tau}^{t}\left|\boldsymbol{w}\left(u, X_{u}\right)\right|^{2} d u\right]-M \delta,
$$

where $M$ is an upper bound for $k$.

To derive a $\mathrm{uBMO}_{\text {loc }}$-estimate, let $L$ be the Lipschitz constant of the function $h$ on $B_{c}$. Since $v \in C_{\mathrm{loc}}^{\left\{\alpha_{n}\right\}}$, for any given $n$, there exists a constant $C_{n}$ such that

$$
\left|\boldsymbol{v}(t, x)-\boldsymbol{v}\left(t^{\prime}, x^{\prime}\right)\right| \leq C_{n} \max \left\{\left|x-x^{\prime}\right|^{\alpha_{n}},\left|t^{\prime}-t\right|^{\alpha_{n} / 2}\right\}
$$

for any $t, t^{\prime} \in[0, T]$ and $\left|x-x^{\prime}\right| \leq n$. The Markov inequality coupled with Lemma 5.1 then imply that

$$
\begin{aligned}
\mathbb{E}_{\tau}[ & \left.h\left(\boldsymbol{v}\left(t, X_{t}\right)\right)-h\left(\boldsymbol{v}\left(t, X_{\tau}\right)\right)\right] \\
& \leq L \mathbb{E}_{\tau}\left[\left|\boldsymbol{v}\left(t, X_{t}\right)-\boldsymbol{v}\left(t, X_{\tau}\right)\right|\right] \\
& \leq \mathbb{E}_{\tau}\left[\left|\boldsymbol{v}\left(t, X_{t}\right)-\boldsymbol{v}\left(t, X_{\tau}\right)\right| \mathbf{1}_{\left|X_{t}-X_{\tau}\right| \leq n}\right]+2\|\boldsymbol{v}\|_{\mathbb{L}^{\infty}} \mathbb{P}_{\tau}\left[\left|X_{t}-X_{\tau}\right|>n\right] \\
& \leq \mathbb{E}_{\tau}\left[\max \left(\left|X_{t}-X_{\tau}\right|^{\alpha_{n}},(t-\tau)^{\alpha_{n} / 2}\right)\right]+(t-\tau)^{1 / 2} \leq_{C} \delta^{\alpha_{n} / 2} .
\end{aligned}
$$

The statement then follows from combining above displayed estimates.

The uniqueness part of the proof is based on a result ([32], Proposition 2.1) of Frei, which in turn extends ([56], Proposition 1) from BSDE whose generator does not depend on $\boldsymbol{y}$ and the terminal condition is small in $\mathbb{L}^{\infty}$-norm, to those whose 
terminal condition is small in the BMO-norm (see, also, [43], Theorem A.1, for a similar result). We now work with $\boldsymbol{f}$ which does not depend on $\boldsymbol{y}$ and derive a consequence of Proposition 5.2 above.

COROllary 5.3. Let $\boldsymbol{F}:[0, T] \times \mathbb{R}^{d} \rightarrow \mathbb{R}^{N}$ be continuous and bounded, and let $\boldsymbol{g} \in C_{\mathrm{loc}}^{\left\{\alpha_{n}\right\}} \cap \mathbb{L}^{\infty}$. The linear system

$$
d \boldsymbol{Y}_{t}=-\boldsymbol{F}\left(t, X_{t}\right) d t+\boldsymbol{Z}_{t} \sigma_{t} d W_{t}, \quad \boldsymbol{Y}_{T}=\boldsymbol{g}\left(X_{T}\right),
$$

admits a solution $(\boldsymbol{v}, \boldsymbol{w})$, which is unique in the class of bounded solutions. Furthermore, $\boldsymbol{v} \in C_{\mathrm{loc}}^{\left\{\alpha_{n}\right\}}$ and $\boldsymbol{w} \in u B M O_{\mathrm{loc}}$.

PROOF. Let $\left\{\boldsymbol{f}^{m}, \boldsymbol{g}^{m}\right\}$ be a sequence of smooth approximations obtained by mollification of the functions $\boldsymbol{F}$ and $\boldsymbol{g}$, respectively. This sequence of approximation does not depend on $(\boldsymbol{y}, \boldsymbol{z})$ and can be constructed so that $\left\|\boldsymbol{f}^{m}\right\|_{\mathbb{L}^{\infty}}+$ $\left\|\boldsymbol{g}^{m}\right\|_{\mathbb{L}^{\infty}} \leq 1+\|\boldsymbol{F}\|_{\mathbb{L}^{\infty}}+\|\boldsymbol{g}\|_{\mathbb{L}^{\infty}}$ for all $m$, with $\left\{\boldsymbol{g}^{m}\right\}$ bounded in $C_{\text {loc }}^{\left\{\alpha_{n}\right\}}$ (cf. Proposition 5.4 below).

Thanks to their boundedness and independence of $z$, these functions are easily seen to satisfy the conditions of Theorem 2.5. In fact, they admit a common $c$-Lyapunov pair for any $c$-indeed, it is enough to choose a quadratic $h$ and largeenough constant $k$. Thanks to the Lipschitz continuity of its coefficients, the equation

$$
d \boldsymbol{Y}_{t}=-\boldsymbol{f}^{m}\left(t, X_{t}\right) d t+\boldsymbol{Z}_{t} \sigma_{t} d W_{t}, \quad \boldsymbol{Y}_{T}=\boldsymbol{g}^{m}\left(X_{T}\right),
$$

admits a continuous Markovian solution $\left(\boldsymbol{v}^{m}, \boldsymbol{w}^{m}\right)$ for each $m$, with $\left\{\boldsymbol{v}^{m}\right\}$ uniformly bounded. Therefore, by Theorem 2.8, there exists a locally Hölderian solution to (5.5), that is, a Markovian solution $(\boldsymbol{v}, \boldsymbol{w})$ with $\boldsymbol{v} \in C_{\mathrm{loc}, b_{0}}^{\left\{\alpha_{n}^{\prime}\right\}}$, for some $b_{0} \in \mathbb{R}^{d}$. Moreover, since a (global) Lyapunov pair $(h, k)$ exists with $k$ bounded, and the Hölder norm of $\boldsymbol{g}$ does not depend on $b_{0}$, the last statement of Theorem 2.5 implies that the Hölder norm of $\boldsymbol{v}$ does not depend on $b_{0}$, that is, $\boldsymbol{v} \in C_{\mathrm{loc}}^{\left\{\alpha_{n}\right\}}$.

It is straightforward to see that this solution is unique in the class of all bounded solutions. Moreover, thanks to the existence of a Lyapunov pair mentioned above, the conditions of Proposition 5.2 are satisfied, and so, $w \in \mathrm{uBMO}_{\mathrm{loc}}$.

To complete the proof of Theorem 2.9, we pick that we pick two bounded- $v$ continuous solutions $(\boldsymbol{v}, \boldsymbol{w})$ and $\left(\boldsymbol{v}^{\prime}, \boldsymbol{w}^{\prime}\right)$. By Remark 2.6 part (2), both of them are locally Hölderian. Since a (global) Lyapunov pair $(h, k)$ exists with $k$ bounded, and the Hölder norm of $\boldsymbol{g}$ does not depend on $b_{0}$, the last statement of Theorem 2.5 implies that the Hölder norms of $\boldsymbol{v}$ and $\boldsymbol{v}^{\prime}$ do not depend on $b_{0}$, that is, $\boldsymbol{v}, \boldsymbol{v}^{\prime} \in C_{\mathrm{loc}}^{\left\{\alpha_{n}\right\}}$. (We can assume, without loss of generality, that they both belong to some $C_{\text {loc }}^{\left\{\alpha_{n}\right\}}$, with the same exponent sequence $\left\{\alpha_{n}\right\}$.) We define $t_{0} \in[0, T]$ by

$$
t_{0}=\inf \left\{t \in[0, T]: \boldsymbol{v}(u, x)=\boldsymbol{v}^{\prime}(u, x) \text { for all } x \in \mathbb{R}^{d}, u \in[t, T]\right\} .
$$


Let us assume-contrary to the conclusion of the theorem-that $t_{0}>0$. When restricted to $\left[0, t_{0}\right]$, both $(\boldsymbol{v}, \boldsymbol{w})$ and $\left(\boldsymbol{v}^{\prime}, \boldsymbol{w}^{\prime}\right)$ are bounded Markovian solutions to (2.2) with the terminal condition $\boldsymbol{g}=\boldsymbol{v}\left(t_{0}, \cdot\right)=\boldsymbol{v}^{\prime}\left(t_{0}, \cdot\right)$. They differ, however, on each interval of the form $\left[t_{0}-\delta, t_{0}\right], \delta>0$.

Let be $\left(\boldsymbol{v}_{f}, \boldsymbol{w}_{f}\right)$, be the unique bounded solution to the auxiliary equation (5.5) on $\left[0, t_{0}\right]$ with $\boldsymbol{F}=\boldsymbol{f}(\cdot, \cdot, 0)$ and $\boldsymbol{g}=\boldsymbol{v}\left(t_{0}, \cdot\right)$. The conditions of Corollary 5.3 above are satisfied, so we have

$$
\lim _{\delta \searrow 0} \sup _{t_{0}-\delta \leq \tau \leq t_{0}} \mathbb{E}_{\tau}\left\|\left[\int_{\tau}^{t_{0}}\left|\boldsymbol{w}_{f}\left(u, X_{u}\right)\right|^{2} d u\right]\right\|_{\mathbb{L}^{\infty}}=0 .
$$

At this point, everything is ready for the application of the aforementioned local uniqueness result of Frei, which we summarize for the reader's convenience: when the quantity

$$
\sup _{t_{0}-\delta \leq \tau \leq t_{0}}\left\|\mathbb{E}_{\tau}\left[\int_{\tau}^{t_{0}}\left|\boldsymbol{w}_{f}\left(u, X_{u}\right)\right|^{2} d u\right]\right\|_{\mathbb{L}^{\infty}},
$$

which is the BMO norm of the terminal condition $\boldsymbol{g}$ on $\left[t_{0}-\delta, t_{0}\right]$, is small, solution $(\boldsymbol{v}, \boldsymbol{w})$ to $(2.2)$ is unique in a class $\mathcal{C}$ of $(\hat{\boldsymbol{v}}, \hat{\boldsymbol{w}})$ with the bmo-norm of $\hat{\boldsymbol{w}}$ on $\left[t_{0}-\delta, t_{0}\right]$, that is, the quantity

$$
\sup _{t_{0}-\delta \leq \tau \leq t_{0}}\left\|\mathbb{E}_{\tau}\left[\int_{\tau}^{t_{0}}\left|\hat{\boldsymbol{w}}\left(u, X_{u}\right) \sigma\left(u, X_{u}\right)\right|^{2} d u\right]\right\|_{\mathbb{L}^{\infty}},
$$

is sufficiently small.

Thanks to (5.7), Frei's result applies when $t \in\left[0, t_{0}\right)$ is chosen close enough to $t_{0}$. By making it even closer, if necessary, we can use Proposition 5.2 to make sure that both of our solutions $(\boldsymbol{v}, \boldsymbol{w})$ and $\left(\boldsymbol{v}^{\prime}, \boldsymbol{w}^{\prime}\right)$ belong to the class $\mathcal{C}$. Therefore, thanks to the fact that $X_{u}$ has a full support under $\mathbb{P}^{t, x}$ for $t<u \leq t_{0}$, we conclude that $\boldsymbol{v}(u, \cdot)=\boldsymbol{v}^{\prime}(u, \cdot)$ for each $t<u \leq t_{0}$ - a contradiction with our definition of $t_{0}$. To show that $\boldsymbol{w}=\boldsymbol{w}^{\prime}$, a.e., we simply appeal to Lemma 4.4 above.

5.3. Proof of Theorem 2.14. Our proof of Theorem 2.14 proceeds in two steps. In the first step, we construct a sequence of Lipschitz approximations to the generator $\boldsymbol{f}$ and the terminal condition $\boldsymbol{g}$, making sure there is enough uniformity for the construction of a uniform Lyapunov pair. Next, we observe that those approximation satisfy the condition $(\mathrm{AB})$ or $(\mathrm{wAB})$, producing a uniform, a priori bound in $\mathbb{L}^{\infty}$. Lastly, we apply the approximation Theorem 2.5 .

5.3.1. Lipschitz approximations. We start by outlining a Lipschitzapproximation procedure that will be used in the sequel. We extend slightly the notation for the class of functions satisfying the condition (BF) from Definition 2.10, by including a general, but small, quadratic term; its significance is explained in 
Remark 2.15 and the additional term corresponds to the "error" in (2.11). If a function $f:[0, T] \times \mathbb{R}^{d} \times \times \mathbb{R}^{N} \times \mathbb{R}^{N \times d} \rightarrow \mathbb{R}^{N}$ admits the following decomposition:

$$
\begin{aligned}
\boldsymbol{f}(t, x, \boldsymbol{y}, \boldsymbol{z})= & \operatorname{diag}(\boldsymbol{z} \mathbf{l}(t, x, \boldsymbol{y}, \boldsymbol{z}))+\mathbf{q}(t, x, \boldsymbol{y}, \boldsymbol{z}) \\
& +\mathbf{s}(t, x, \boldsymbol{y}, \boldsymbol{z})+\mathbf{e}(t, x, \boldsymbol{y}, \boldsymbol{z})+\mathbf{k}(t, x),
\end{aligned}
$$

where I, q, s, k satisfy the conditions of Definition 2.10 , and for each $n \in \mathbb{N}$ we have

$$
\begin{aligned}
& |\mathbf{e}(t, x, \boldsymbol{y}, \boldsymbol{z})| \leq \epsilon_{n}\left(1+|z|^{2}\right) \\
& \quad \text { for some } \epsilon_{n}>0 \text { and all }(t, x, z) \in[0, T] \times B_{n}\left(b_{0}\right) \times \mathbb{R}^{N} \times \mathbb{R}^{N \times d},
\end{aligned}
$$

then we say that $\boldsymbol{f}$ satisfies the approximate condition $(B F)$, and write $\boldsymbol{f} \in$ $\mathbf{B F}_{e}\left(\left\{C_{n}\right\},\left\{\kappa_{n}\right\},\left\{q_{n}\right\},\left\{\varepsilon_{n}\right\}\right)$.

As Proposition 5.4 below shows, a pleasant feature of the condition (BF) (and its approximate version) is that it allows for approximation by more regular functions, in a uniform way. More precisely, $f$ can be approximated by a sequence of regular functions $\left\{\boldsymbol{f}^{m}\right\}$, such that, even though the functions $\mathbf{I}, \mathbf{q}, \mathbf{s}, \mathbf{k}$ and $\mathbf{e}$ in the decomposition of $\boldsymbol{f}^{m}$ may depend on $m$, the constant sequences $\left(\left\{C_{n}\right\},\left\{\kappa_{n}\right\},\left\{q_{n}\right\},\left\{\varepsilon_{n}\right\}\right)$ do not. This uniformity is essential to construct a sequence of universal Lyapunov functions $\left\{h_{n}\right\}$ for the approximation sequence $\left\{f^{m}\right\}$.

PROPOSITION 5.4 [Approximations preserving the approximate condition $(\mathrm{BF})]$.

1. For each $\boldsymbol{g} \in C_{\mathrm{loc}}^{\left\{\alpha_{n}\right\}}$, then there exists a sequence $\left\{\boldsymbol{g}^{m}\right\}$, bounded in $C_{\mathrm{loc}}^{\left\{\alpha_{n}\right\}}$, such that each $\boldsymbol{g}^{m}$ is Lipschitz (globally in all arguments) and $\boldsymbol{g}^{m} \rightarrow \boldsymbol{g}$ everywhere.

2. There exists a constant $M$, which depends only on $d$ and $N$ such that for each $\boldsymbol{f} \in \mathbf{B F}_{e}\left(\left\{C_{n}\right\},\left\{\kappa_{n}\right\},\left\{q_{n}\right\},\left\{\varepsilon_{n}\right\}\right)$, there exists a sequence $\left\{\boldsymbol{f}^{m}\right\}$ and a subquadratic sequence $\left\{\kappa_{n}^{\prime}\right\}$ (as in Definition 2.10) such that:

(a) $\boldsymbol{f}^{m} \in \mathbf{B F}_{e}\left(M C_{n}, \kappa_{n}^{\prime}, q_{n}, M \varepsilon_{n}\right)$ and is globally Lipschitz in all of its arguments,

(b) $\boldsymbol{f}^{m} \rightarrow \boldsymbol{f}$ pointwise, locally uniformly in $\boldsymbol{z}$, and

(c) $\left\|\boldsymbol{f}^{m}(\cdot, \cdot, 0)\right\|_{\mathbb{L}^{q_{n}}\left([0, T] \times B_{n}\left(b_{0}\right)\right)}$ is bounded uniformly in $m$, for each $n \in \mathbb{N}$.

PROOF. The idea is to mollify using smooth kernels with a compact support and linearize the tails of the quadratic parts. In this spirit, we define the $C^{\infty}\left(\mathbb{R}^{d}\right)$ function

$$
\eta(x)=C e^{\frac{1}{|x|^{2}-1}} \mathbf{1}_{|x|<1},
$$

with the $C$ is chosen so that $\int \eta(x) d x=1$. We use the same notation $\eta$ (and the same formula) for its $C^{\infty}(\mathbb{R})$ and $C^{\infty}\left(\mathbb{R}^{N \times d}\right)$ versions. 
For each $m \in \mathbb{N}$, we set $\eta^{m}(x)=m^{d} \eta(m x)$ and $\eta^{m}(t, x, z)=m^{1+d+N \times d} \times$ $\eta(m t) \eta(m x) \eta(m z)$, and use the standard notation for mollification, namely,

$$
(g * \eta)(x)=\int \boldsymbol{g}(x-\bar{x}) \eta(\bar{x}) d \bar{x}
$$

as well as for its $\eta(t, x, z)$-version. We refer the reader to [30], Appendix C.4, Theorem 6, for standard properties of mollification.

We also define the partial-truncation function $\Pi^{m}(\boldsymbol{w})=\frac{|\boldsymbol{w}| \wedge m}{|\boldsymbol{w}|} \boldsymbol{w}$, with $\Pi^{m}(0)=0$; clearly, $\Pi^{m}$ is Lipschitz and $\left|\Pi^{m}(\boldsymbol{w})\right|=|\boldsymbol{w}| \wedge m$. Most of the approximations in this proof will be of the form

$$
\boldsymbol{g}^{m}(x)=\left(\boldsymbol{g} * \eta^{m}\right)\left(\Pi^{m}(x)\right)
$$

in the $\mathbb{R}^{d}$ case and

$$
\boldsymbol{f}^{m}(t, x, z)=\left(\boldsymbol{f} * \eta^{m}\right)\left(t, \Pi^{m}(x), \Pi^{m}(\boldsymbol{z})\right)
$$

in the $\mathbb{R}^{1+d+N \times d}$-case after extending the domain of $\boldsymbol{f}$ via $\boldsymbol{f}(t, x, \boldsymbol{z})=\boldsymbol{f}(0, x, \boldsymbol{z})$ for $t<0$, and $f(t, x, z)=f(T, x, z)$ when $t>T$. The same, superscript- $m$, notation will be used without explicit mention, when this operation is applied to other functions below.

(1) For $\boldsymbol{g} \in C_{\mathrm{loc}, b_{0}}^{\left\{\alpha_{n}\right\}}$, one easily checks that each $\boldsymbol{g}^{m}$ is Lipschitz, bounded, the sequence $\left(\boldsymbol{g}^{m}\right)$ is bounded in $C_{\mathrm{loc}, b_{0}}^{\left\{\alpha_{n}\right\}}$, and the convergence $\boldsymbol{g}^{m} \rightarrow \boldsymbol{g}$ follows from the standard properties of mollification.

(2) Given a function $\boldsymbol{f}$ satisfying the assumptions in part (2), since the convolution $\boldsymbol{f} * \eta^{m}$ is smooth, and $\Pi^{m}$ is Lipschitz and bounded, each approximation $\boldsymbol{f}^{m}$ is globally Lipschitz in all of its variables. Furthermore, when $\boldsymbol{z}^{m} \rightarrow \boldsymbol{z}$, we have $\Pi^{m}\left(z^{m}\right)=z^{m}$ for sufficiently large $m$ and, so, using the properties that mollifications of a continuous function converge locally uniformly, we have $f^{m} \rightarrow f$ locally uniformly in $z$.

To verify the condition $\mathbf{B F} \mathbf{F}_{e}$ for $\boldsymbol{f}^{m}$, we fix $n \in \mathbb{N}$, and start with the quadratictriangular component $\mathbf{q}$. Thanks to the fact that $\eta$ is of compact support, all components $\mathbf{q}^{m, i}$ of the approximation $\boldsymbol{q}^{m}$ have the following property:

$$
\begin{aligned}
\left|\mathbf{q}^{m, i}(t, x, z)\right| & \leq C_{n}\left(2+\sum_{j=1}^{i}\left|z^{j}\right|^{2}\right) \\
& \leq 2 C_{n}\left(1+\sum_{j=1}^{i}\left|z^{j}\right|^{2}\right) \quad \text { on }[0, T] \times B_{n} \times \mathbb{R}^{N \times d} .
\end{aligned}
$$

Therefore, $\mathbf{q}^{m}$ is quadratic-triangular as well, with $C_{n}^{\prime}=2 C_{n}$. A similar argument can be applied to e. For k, it follows from [30], Appendix C.4, Theorem 6(iv), that $\mathbf{k}^{m}$ converges to $\mathbf{k}$ in $\mathbb{L}^{q_{n}}\left([0, T] \times B_{n}\right)$ for each $n$; in particular, the sequence 
$\left\|\mathbf{k}^{m}\right\|_{\mathbb{L}^{q_{n}}\left([0, T] \times B_{n}\right)}$, which differs from $\left\|\boldsymbol{f}^{m}(\cdot, \cdot, 0)\right\|_{\mathbb{L}^{q_{n}}\left([0, T] \times B_{n}\right)}$ only by a constant, is bounded in $m$.

For $\mathbf{I}$, a direct approximation of $\mathbf{d}(t, x, z)=\operatorname{diag}(z \mathbf{I}(t, x, z))$ does not produce the function in the same class; it needs an adjustment by a subquadratic term. To see that, we note that

$$
\mathbf{d}^{m}(t, x, \boldsymbol{z})=\operatorname{diag}\left(\boldsymbol{z} \mathbf{I}^{m}(t, x, \boldsymbol{z})\right)-\mathbf{L}^{m}(t, x, \boldsymbol{z}),
$$

where $\mathbf{d}^{m}(t, x, \boldsymbol{z})=\left(\mathbf{d} * \eta^{m}\right)\left(t, \Pi^{m}(x), \Pi^{m}(\boldsymbol{z})\right)$ and

$$
\begin{aligned}
\left(\mathbf{L}^{i}\right)^{m}(t, x, \boldsymbol{z}) & =\sum_{j=1}^{d}\left(\mathbf{I}_{j i} * \hat{\eta}_{i j}^{m}\right)\left(t, \Pi^{m}(x), \Pi^{m}(\boldsymbol{z})\right) \quad \text { and } \\
\left(\hat{\eta}^{m}\right)_{i j}(t, x, \boldsymbol{z}) & =z_{i j} \eta^{m}(t, x, \boldsymbol{z}) .
\end{aligned}
$$

The function $\mathrm{I}^{m}$ grows at most linearly, with the constant $C_{n}^{\prime}$ bounded from above by $C_{n}$ multiplied by a constant which depends only on $d$ and $N$. On the other hand, the components of $\mathbf{L}^{m}$ are mollifications of linearly-growing functions by kernels $\hat{\eta}_{i j}^{m}$, all of which are dominated by $\eta^{m}$ in absolute value. Therefore, the functions $\mathbf{L}^{m}$ are of subquadratic growth, uniformly in $m$.

The subquadratic growth of $\mathbf{s}$ ensures the same property for $\mathbf{s}^{m}$, uniformly in $m$, perhaps with a different growth bound $\kappa_{n}^{\prime}$. Therefore, each $\boldsymbol{f}^{m}$ admits a decomposition as in (5.8) into the functions $\mathbf{d}^{m}+\mathbf{L}^{m}, \boldsymbol{q}^{m}, \boldsymbol{s}^{m}-\mathbf{L}^{m}, \mathbf{e}^{m}$ and $\mathbf{k}^{m}$, which have all the required properties.

5.3.2. Existence of Lyapunov pairs. Proposition 5.5 below confirms Proposition 2.11 and Remark 2.15 part (1). Its proof is partially based on a construction in [7], Proposition 3.1, page 174.

Proposition 5.5. Let $\left\{\boldsymbol{f}^{m}\right\}$ be a sequence in $\mathbf{B F}_{e}\left(\left\{C_{n}\right\},\left\{\kappa_{n}\right\},\left\{q_{n}\right\},\left\{\varepsilon_{n}\right\}\right)$ with $\left\|\boldsymbol{f}^{m}(\cdot, \cdot, 0)\right\|_{\mathbb{L}^{q_{n}}\left([0, T] \times B_{n}\left(b_{0}\right)\right)}$ bounded, for each $n \in \mathbb{N}$. Then, for each sequence $\left\{c_{n}\right\}$ of positive numbers, there exists a sequence $\left\{\bar{\varepsilon}_{n}\right\}$ in $(0, \infty)$ such that if $\varepsilon_{n} \leq \bar{\varepsilon}_{n}$ for all $n$, there exist families $\left\{h_{n}\right\}$ and $\left\{k_{n}^{m}\right\}$ such that, for each $m,\left(\left\{h_{n}\right\},\left\{k_{n}^{m}\right\}\right)$ is a local $\left\{c_{n}\right\}$-Lyapunov pair for $\boldsymbol{f}^{m}$. In particular, when $\left\{C_{n}\right\},\left\{\kappa_{n}\right\},\left\{q_{n}\right\}$ and $\left\{\varepsilon_{n}\right\}$ are constants in $n$, there exists a c-Lyapunov pair for $\boldsymbol{f}$, for any $c>0$.

ProOF. We restrict the spatial domain to $B_{n}\left(b_{0}\right)$ and suppress the subscript $n$ throughout the proof. When $\left\{C_{n}\right\},\left\{\kappa_{n}\right\},\left\{q_{n}\right\}$ and $\left\{\varepsilon_{n}\right\}$ are constants in $n$, the spatial domain is $\mathbb{R}^{d}$. Since $\left\{\boldsymbol{f}^{m}\right\}$ satisfies the approximate condition (BF) with uniform growth sequences $\left\{C_{n}\right\},\left\{\kappa_{n}\right\},\left\{q_{n}\right\}$ and $\left\{\varepsilon_{n}\right\}$, we suppress the superscript $m$ as well. For $\boldsymbol{y}=\left(y_{1}, \ldots, y_{N}\right) \in \mathbb{R}^{N}$ and $k=1, \ldots, N$, we define

$$
G_{k}(\boldsymbol{y})=\cosh \left(\alpha_{k} y_{k}\right) \quad \text { and } \quad S_{k}(\boldsymbol{y})=\sinh \left(\alpha_{k} y_{k}\right),
$$


with $\alpha_{1}, \ldots, \alpha_{N}>0$ to be determined later. Recursively, we set

$$
H_{N+1}=0 \quad \text { and } \quad H_{k}=\exp \left(G_{k}+H_{k+1}\right) \quad \text { for } k=1, \ldots, N,
$$

as well as

$$
P_{0}=1, \quad P_{k}=\prod_{i=1}^{k} H_{i}, \quad k=1, \ldots, N,
$$

noting that $1 \leq P_{1} \leq P_{2} \leq \cdots \leq P_{N}$. The (linear combinations) of functions $G_{k}$ and $S_{k}$ play the role of $\beta$, and $H_{k}$ the role $X_{v}$, in the notation of [7], equation (3.6), page 174 . With $A_{i}=\alpha_{i} S_{i} P_{i}$, we compute

$$
D_{i} H_{k}=A_{i} P_{k-1}^{-1} \mathbf{1}_{\{i \geq k\}},
$$

where $D_{i}$ stands for $\frac{\partial}{\partial y^{i}}$, so that

$$
D_{j} P_{i}=P_{i} \sum_{k=1}^{i}\left(H_{k}\right)^{-1} D_{j} H_{k}=P_{i} A_{j} \sum_{k=1}^{i \wedge j}\left(P_{k}\right)^{-1} \text {. }
$$

Setting $h=H_{1}$ and $\tilde{A}_{i}=\alpha_{i}^{2} G_{i} P_{i}$, for $1 \leq i, j \leq N$, we obtain

$$
D_{i} h=A_{i} \quad \text { and } \quad D_{i j} h=\tilde{A}_{i} \mathbf{1}_{\{i=j\}}+A_{i} A_{j} \sum_{k=1}^{i \wedge j} P_{k}^{-1} .
$$

To prove (2.4), we pick an $N \times d$-matrix $z$ and set $\zeta^{i}=z^{i} \sigma$ where $z^{i}$ is the $i$ th row of $z$, so that $\frac{1}{\Lambda}\left|z^{i}\right|^{2} \leq\left|\zeta^{i}\right|^{2} \leq \Lambda\left|z^{i}\right|^{2}$. Thanks to (5.10), we obtain

$$
D^{2} h:\langle z, z\rangle_{a}=\sum_{i j} D_{i j} h \zeta^{i}\left(\zeta^{j}\right)^{\top}=\sum_{i=1}^{N} \tilde{A}_{i}\left|\zeta^{i}\right|^{2}+\sum_{i=1}^{N} P_{i}^{-1}\left|\boldsymbol{\eta}_{i}(\zeta)\right|^{2},
$$

where $\eta^{i}(\zeta)=\sum_{j=i}^{N} A_{j} \zeta^{j}$.

To deal with the $D h \boldsymbol{f}$-part in (2.4), we consider various constituents in (2.8) separately. We reuse the letter $C$ for any constant-possibly differing from place to place-which depends only on the sequences $\left\{C_{n}\right\}$ and $\left(\kappa_{n}\right)$ from statement, or the universal constants.

- The Quadratic-Linear part: Let $\lambda^{i}$ denote the $i$ th row of the matrix $\mathbf{I}^{\top} \sigma^{-1}$, so that

$$
\operatorname{diag}(z \mathbf{I})_{i}=(z \mathbf{I})_{i i}=\zeta^{i}\left(\lambda^{i}\right)^{\top} .
$$

If we extend the definition of $\boldsymbol{\eta}=\boldsymbol{\eta}(\zeta)$ and $\lambda$ by setting $\eta^{n+1}=\lambda^{0}=\mathbf{0}$, "summation by parts" implies that

$$
\sum_{i=1}^{N} A_{i} \zeta^{i}\left(\lambda^{i}\right)^{\top}=\sum_{i=1}^{N}\left(\eta^{i}-\eta^{i+1}\right)\left(\lambda^{i}\right)^{\top}=\sum_{i=1}^{N} \eta^{i}\left(\lambda^{i}-\lambda^{i-1}\right)^{\top} .
$$


The fact that $|\mathbf{I}| \leq C(1+|z|)$ and Young's inequality yield

$$
\begin{aligned}
\operatorname{Dh} \operatorname{diag}(\boldsymbol{z l}) & =\sum_{i=1}^{N} \boldsymbol{\eta}^{i}\left(\lambda^{i}-\lambda^{i-1}\right)^{\top} \leq \sum_{i=1}^{N} C\left|\boldsymbol{\eta}^{i}\right|(1+|\zeta|) \\
& \leq \sum_{i=1}^{N} C P_{i}\left(1+|\zeta|^{2}\right)+\frac{1}{2} \sum_{i=1}^{N} P_{i}^{-1}\left|\eta^{i}(\zeta)\right|^{2} .
\end{aligned}
$$

- The Quadratic-Triangular part:

$$
D h \mathbf{q}=\sum_{i=1}^{N} A_{i} \mathbf{q}^{i} \leq C \sum_{j=1}^{N}\left(1+\left|\zeta^{j}\right|^{2}\right) \sum_{i=j}^{N}\left|A_{i}\right| \leq C \sum_{j=1}^{N}\left(1+\left|\zeta^{j}\right|^{2}\right) \sum_{i=j}^{N}\left|A_{i}\right| .
$$

- Choosing constants $\alpha_{1}, \ldots, \alpha_{N}$ : The inequality $\sum_{j=1}^{N} P_{i} \leq N \sum_{j=i}^{N} P_{j}$ is valid for each $i$, so

$$
\begin{aligned}
\frac{1}{2} D^{2} h & :\langle\boldsymbol{z}, \boldsymbol{z}\rangle_{a}-\operatorname{Dh}(\operatorname{diag}(\boldsymbol{z} \mathbf{l})+\mathbf{q}) \\
& \geq \sum_{i=1}^{N}\left(\frac{1}{2} \tilde{A}_{i}-C \sum_{j=i}^{N}\left(P_{j}+\left|A_{j}\right|\right)\right)\left|\zeta^{i}\right|^{2}-C \sum_{j=i}^{N}\left(P_{j}+\left|A_{j}\right|\right) \\
& \geq \frac{1}{\Lambda} \sum_{i=1}^{N}\left(\frac{1}{2} \tilde{A}_{i}-C \sum_{j=i}^{N}\left(P_{j}+\left|A_{j}\right|\right)\right)\left|z^{i}\right|^{2}-C \sum_{j=i}^{N}\left(P_{j}+\left|A_{j}\right|\right) .
\end{aligned}
$$

The choice $\alpha_{N} \geq 2 C+1$, together with $G_{N} \geq 1$ and $\left|S_{N}\right| \leq G_{N}$ yields $\frac{1}{2} \tilde{A}_{N}>$ $C\left(P_{N}+\left|A_{N}\right|\right)$. For $i \leq N-1$, we have

$$
\frac{1}{2} \tilde{A}_{i}-C \sum_{j=i}^{N}\left(P_{j}+\left|A_{j}\right|\right) \geq\left[\frac{1}{2} \alpha_{i}^{2}-C\left(1+\alpha_{i}\right)\right] G_{i} P_{i}-\sum_{j=i+1}^{N}\left(P_{j}+\left|A_{j}\right|\right) .
$$

Next, we observe the fact that $\sum_{j=i+1}^{N}\left(P_{j}+\left|A_{j}\right|\right)$ depends only on $\alpha_{i+1}, \ldots, \alpha_{N}$, and is bounded on the set $[-c, c]^{N}$ as a function of $y^{i+1}, \ldots, y^{N}$. Therefore, we can choose a sufficiently large $\alpha_{i}$ so that the left-hand side of the previous inequality is positive, and continue this process recursively down to $i=1$. This way, we obtain a constant $C_{0}$, depending on $C, c$ and the universal constants, so that

$$
\frac{1}{2} D^{2} h:\langle z, z\rangle_{a}-D h(\operatorname{diag}(z \mathbf{I})+\mathbf{q}) \geq C_{0}|z|^{2}-C_{0} .
$$

- The Subquadratic part: With $\alpha_{1}, \ldots, \alpha_{N}$ now fixed, $\sum_{i}\left|A_{i}\right|$ is bounded on $[-c, c]^{N}$, as a function of $\boldsymbol{y}$. Therefore, $\varepsilon_{0}=C_{0} / \sup _{[-c, c]^{N}}\left(\sum_{i}\left|A_{i}\right|\right)>0$. With $\varepsilon$ as in (2.11) assumed to be smaller than $\varepsilon_{0}$, we pick $\varepsilon^{\prime}<\varepsilon_{0}-\varepsilon$ and set

$$
\kappa^{*}=\sup _{\iota \geq 0}\left(\kappa(\iota)-\varepsilon^{\prime} \iota\right) .
$$


The sublinear growth of $\kappa$ ensures that $\kappa^{*}$ is well-defined in $[0, \infty)$ and

$$
D h \mathbf{s}=\sum_{i=1}^{n} A_{i} s^{i} \leq \sum_{i=1}^{N}\left|A_{i}\right| \kappa\left(|z|^{2}\right) \leq \frac{C_{0}}{\varepsilon_{0}}\left(\kappa^{*}+\varepsilon^{\prime}|z|^{2}\right)=\frac{C_{0} \varepsilon^{\prime}}{\varepsilon_{0}}|z|^{2}+\frac{C_{0}}{\varepsilon_{0}} \kappa^{*} .
$$

Lastly, we combine all of the above estimates to obtain

$$
\begin{aligned}
\frac{1}{2} D^{2} h:\langle\boldsymbol{z}, \boldsymbol{z}\rangle_{a}-D h \boldsymbol{f} & \geq \frac{1}{2} D^{2} h:\langle\boldsymbol{z}, \boldsymbol{z}\rangle_{a}-D h(\operatorname{diag}(\boldsymbol{z} \mathbf{l})+\mathbf{q}+\mathbf{s}+\mathbf{k})-\varepsilon|D h||\boldsymbol{z}|^{2} \\
& \geq\left(C_{0} \frac{\varepsilon_{0}-\varepsilon^{\prime}}{\varepsilon_{0}}-\varepsilon|D h|\right)|z|^{2}-C_{0}-C_{0} \varepsilon_{0} \kappa^{*}-D h \mathbf{k} .
\end{aligned}
$$

Since $\varepsilon|D h| \leq \varepsilon C_{0} / \varepsilon_{0}$, it suffices to define $k=C_{0}+C_{0} \varepsilon_{0} \kappa^{*}+C_{1}|\mathbf{k}|$, for some $C_{1} \geq|D h|$, and, if necessary, scale both $h$ and $\varepsilon_{0}$ (yielding $\bar{\varepsilon}$ ) to make the coefficient in front of $|z|^{2}$ equal to 1 .

5.3.3. Conclusion of the Proof of Theorem 2.14. Let $g$ and $\boldsymbol{f}$ be two functions which satisfy the conditions of Theorem 2.14, namely $g$ is in $C_{\text {loc, } b_{0}}^{\left\{\alpha_{n}\right\}}$ and of subquadratic growth, $\boldsymbol{f}$ satisfies the conditions (AB) and the approximate condition (BF). We start by picking a sequence $\left(\boldsymbol{f}^{m}, \boldsymbol{g}^{m}\right)$ of Lipschitz approximations constructed in Proposition 5.4. Thanks to the Lipschitz property of all ingredients, each approximate system (2.5) admits a continuous Markovian solution $\left(\boldsymbol{v}^{m}, \boldsymbol{w}^{m}\right)$ (see, e.g., [27], Theorem 4.1 and Corollary 4.1). Moreover, the Lipschitz approximations $\boldsymbol{f}^{m}$ satisfy the condition (AB), of Definition 2.12, possibly with the same positively-spanning set $\boldsymbol{a}_{1}, \ldots, \boldsymbol{a}_{K}$, and a possibly different, but $m$-independent, $\mathbb{L}^{1}$-function $l$. The existence of the latter-as the supremum of a family of finer and finer mollifications of an integrable function-is guaranteed by the HardyLittlewood maximal theorem. Similarly, the approximations $\boldsymbol{g}^{m}$ of the terminal condition $\boldsymbol{g}$ satisfy the inequality $\left|\boldsymbol{g}^{m}(x)\right| \leq \zeta(x)$ uniformly in $m$, for some smooth function $\zeta$ with $\lim _{|x| \rightarrow \infty} \zeta(x) /|x|^{2}=0$.

Let us first show that each $\boldsymbol{v}^{m}$ is bounded. Let $\boldsymbol{a}_{1}, \ldots, \boldsymbol{a}_{K}$ be a positive spanning set from condition (AB). Given $k \in\{1, \ldots, K\}$, we consider the following quadratic BSDE:

$$
d \bar{Y}_{t}^{k, m}=-\left[\ell(t)+\frac{1}{2}\left|\overline{\boldsymbol{Z}}_{t}^{k, m}\right|^{2}\right] d t+\overline{\boldsymbol{Z}}_{t}^{k, m} d W_{t}, \quad \bar{Y}_{T}^{k, m}=\boldsymbol{a}_{k}^{\top} \mathbf{1}_{N} \boldsymbol{g}^{m}\left(X_{T}\right),
$$

where $\mathbf{1}_{N}$ denotes the vector of $1 \mathrm{~s}$ in $\mathbb{R}^{N}$. Since $\boldsymbol{g}^{m}$ is bounded by its construction in Proposition 5.4, the previous BSDE admits a bounded solution $\left(\bar{Y}^{k, m}, \overline{\boldsymbol{Z}}^{k, m}\right)$. Recall that $\left(\boldsymbol{v}^{m}, \boldsymbol{w}^{m}\right)$ is a Markovian solution to a Lipschitz BSDE. The comparison theorem for Lipschitz BSDEs (see, e.g., [27], Theorem 2.2, whose proof only needs one generator to be Lipschitz) implies that $\boldsymbol{a}_{k}^{\top} \boldsymbol{v}^{m}(\cdot, X) \leq \bar{Y}^{k, m}$, hence $\boldsymbol{a}_{k}^{\top} \boldsymbol{v}^{m}(\cdot, X)$ is bounded from above. It remains to use the following fact: a sequence $\left\{\boldsymbol{v}^{m}\right\}$ in $\mathbb{R}^{N}$ for which the sequence $\left\{\boldsymbol{a}^{\top} \boldsymbol{v}^{m}\right\}$ is bounded from above 
for each $\boldsymbol{a}$ in some positive spanning set of $\mathbb{R}^{N}$, is itself bounded in $\mathbb{R}^{N}$. Indeed, since the $i$ th canonical base $\mathfrak{e}_{i}$ for $\mathbb{R}^{N}$ can be positively spanned by $\mathfrak{e}_{i}=$ $\lambda_{1}^{i} a_{1}+\cdots+\lambda_{K}^{i} a_{K}$, we have that the $i$ th component of $\boldsymbol{v}^{m}$ has the decomposition $\mathfrak{e}_{i}^{\top} \boldsymbol{v}^{m}=\lambda_{1}^{i} a_{1}^{\top} \boldsymbol{v}^{m}+\cdots+\lambda_{K}^{i} a_{K}^{\top} \boldsymbol{v}^{m}$, which is bounded from above. Similarly, the argument applied to $-\mathfrak{e}_{i}$ also shows that the $i$ th component of $\boldsymbol{v}^{m}$ is bounded from below.

We now show $\left\{\boldsymbol{v}^{m}\right\}$ is bounded uniformly in compacts of $[0, T] \times \mathbb{R}^{N}$. Given $k \in\{1, \ldots, K\}$, we define

$$
e_{k}^{m}(t, x)=\exp \left(\boldsymbol{a}_{k}^{\top} \boldsymbol{v}^{m}(t, x)-\int_{t}^{T} l(s) d s\right) .
$$

A direct computation yields that for each $(t, x)$, the drift term in the $\mathbb{P}^{t, x}$ semimartingale decomposition of the process $e_{k}^{m}(\cdot, X)$ is given as the integral of

$$
e_{k}^{m}\left(s, X_{s}\right)\left(-\boldsymbol{a}_{k}^{\top} \boldsymbol{f}^{m}\left(s, X_{s}, \boldsymbol{Z}_{s}^{m}\right)+l(s)+\frac{1}{2}\left|\boldsymbol{a}_{k}^{\top} \boldsymbol{Z}_{s}^{m}\right|^{2}\right) .
$$

Therefore, by the condition $(\mathrm{AB})$, the process $e_{k}^{m}(\cdot, X)$ is a nonnegative local submartingale. Thanks to the boundedness of $\boldsymbol{v}^{m}$, it is, in fact, a uniformly bounded submartigale and we can use the Markov property to conclude that

$$
e_{k}^{m}(t, x) \leq \bar{e}(t, x) \quad \text { where } \bar{e}_{k}(t, x):=\mathbb{E}^{t, x}\left[\exp \left(\boldsymbol{a}_{k}^{\top} \mathbf{1}_{N} \zeta\left(X_{T}\right)\right)\right]
$$

Smoothness and the subquadratic growth of $\zeta$ imply that the function $\bar{e}_{k}$ is smooth (see [34], Theorem 12, page 25). We then conclude that the sequence $\left\{e_{k}^{m}\right\}$-and, therefore, also, $\left\{\boldsymbol{a}_{k}^{\top} \boldsymbol{v}^{m}\right\}$-is bounded from above on compact subsets of $[0, T] \times$ $\mathbb{R}^{d}$, uniformly in $m$. Since $\left\{\boldsymbol{a}_{k}\right\}$ positively span $\mathbb{R}^{N},\left\{\boldsymbol{v}^{m}\right\}$ is uniformly bounded on the same compact subset as well.

When $g$ is bounded, but only the weaker version (wAB) of the condition (AB) is satisfied, we have from the construction of $\boldsymbol{f}^{m}$ that

$$
\begin{aligned}
\boldsymbol{a}_{k}^{\top} \boldsymbol{f}^{m}(t, x, \boldsymbol{y}, \boldsymbol{z}) \leq & l(t)+\frac{1}{2}\left|\boldsymbol{a}_{k}^{\top} \boldsymbol{z}\right|^{2} \\
& +\boldsymbol{a}_{k}^{\top} \boldsymbol{z} \boldsymbol{L}_{k}^{m}(t, x, \boldsymbol{z})-\boldsymbol{a}_{k}^{\top}\left(\boldsymbol{L}_{k} * \hat{\eta}^{m}\right)\left(t, \Pi^{m}(x), \Pi^{m}(\boldsymbol{z})\right),
\end{aligned}
$$

for any $m \in \mathbb{N},(t, x) \in[0, T] \times \mathbb{R}^{d}$, and $\boldsymbol{z} \in \mathbb{R}^{N \times d}$. Here, $\boldsymbol{L}_{k}^{m}$ is defined similarly as in (5.9), $\hat{\eta}^{m}(t, x, z)=\boldsymbol{z} \eta^{m}(t, x, z)$. Since the supp $\eta^{m} \subseteq B_{1 / m}$ and $\boldsymbol{L}_{k}$ has at most linear growth, there exists a constant $C$ such that

$$
\left|\boldsymbol{a}_{k}^{\top}\left(L_{k} * \hat{\eta}^{m}\right)\left(t, \Pi^{m}(x), \Pi^{m}(z)\right)\right| \leq_{C} \frac{1}{m}(1+m \wedge|z|) \leq C \quad \text { for all } m .
$$

Combining the previous two estimates, we have

$$
\boldsymbol{a}_{k}^{\top} \boldsymbol{f}^{m}(t, x, \boldsymbol{y}, \boldsymbol{z}) \leq l(t)+\frac{1}{2}\left|\boldsymbol{a}_{k}^{\top} \boldsymbol{z}\right|^{2}+\boldsymbol{a}_{k}^{\top} \boldsymbol{z} \boldsymbol{L}_{k}^{m}(t, x, \boldsymbol{z}),
$$

for a different $l \in \mathbb{L}^{1}[0, T]$. 
Argue as before that the drift of the process $e_{k}^{m}(\cdot, X)$ is given as the integral of

$$
e_{k}^{m}\left(u, X_{u}\right)\left(p_{k}^{m}\left(u, X_{u}\right)-\boldsymbol{a}_{k}^{\top} \boldsymbol{Z}_{u}^{m} \boldsymbol{L}_{k}^{m}\left(u, X_{u}, \boldsymbol{Z}_{u}^{m}\right)\right),
$$

for some functions $p_{k}^{m} \geq 0$, while its martingale-part admits the $d W$-integrand of the form

$$
e_{k}^{m}\left(u, X_{u}\right) \boldsymbol{a}_{k}^{\top} \boldsymbol{Z}_{u}^{m}
$$

Boundedness of $e_{k}^{m}$ and $\boldsymbol{L}_{k}^{m}$ allows us to conclude that $e_{k}^{m}(\cdot, X)$ is a submartingale under an equivalent measure (given as the Girsanov transformation with drift $-\boldsymbol{L}_{k}^{m}$ ), with the terminal value bounded uniformly in $m$. The rest is as before, and leads to a similar conclusion, except that now the boundedness is uniform in $(t, x)$.

Moreover, using only the function $l$ and the positive spanning set $\boldsymbol{a}_{1}, \ldots, \boldsymbol{a}_{K}$ of condition (wAB), we well as the $\mathbb{L}^{\infty}$-bounds on $\boldsymbol{g}$, one can produce an a priori bound $c$ on $\left\{\boldsymbol{v}^{m}\right\}$. This way we obtain a sequence-namely $\left\{c_{n}\right\}$ with each $c_{n}=c$-independently of the other constants $\left\{C_{n}\right\},\left\{q_{n}\right\}$ and $\left\{\kappa_{n}\right\}$ appearing in the approximate condition (BF). This way, we can avoid circularity in the definition the sequence $\left\{\bar{\varepsilon}_{n}\right\}$ of Proposition 5.5, that enforces the "smallness" condition on the "error" term in the (BF)-decomposition of $f$.

Whether $\boldsymbol{g}$ is bounded or unbounded, we have produced a sequence $\left\{c_{n}\right\}$ of a priori bounds that can be used together with the sequences $\left\{\boldsymbol{f}^{m}\right\}$ and $\left\{\boldsymbol{g}^{m}\right\}$ in Proposition 5.5 to construct local $\left\{c_{n}\right\}$-Lyapunov pairs $\left\{h_{n}, k_{n}^{m}\right\}$ for $\boldsymbol{f}^{m}$ with uniformly $\mathbb{L}^{q_{n}}$-bounded $k$-parts. By Theorem 2.8, this is enough to guarantee the existence of locally Hölderian solution $(\boldsymbol{v}, \boldsymbol{w})$.

To establish uniqueness, we note that the sequence $\left\{k_{n}^{m}\right\}$ from Proposition 5.5 will be constant (both in $n$ and $m$ ) under the condition (a) of Theorem 2.14. Moreover, due to the absence of dependence on $n$ in (b), a $c$-Lyapunov pair $(h, k)$ with constant $k$ can be constructed for any $c$. The fact that any bounded continuous solution is a priori bounded by the constant $c$ constructed above, together with the local Lipschitz condition in (c), is enough to apply the abstract uniqueness result Theorem 2.9, part (2).

\subsection{Proofs for examples.}

5.4.1. Proof of Theorem 3.1. Consider the following system of BSDE:

$$
d \tilde{\boldsymbol{Y}}_{t}=\tilde{\boldsymbol{f}}\left(t, X_{t}, \tilde{\boldsymbol{Z}}_{t}\right) d t+\tilde{\boldsymbol{Z}}_{t} \sigma\left(t, X_{t}\right) d W_{t}, \quad \tilde{Y}_{T}=\boldsymbol{g}\left(X_{T}\right),
$$

where

$$
\begin{aligned}
\tilde{\boldsymbol{f}}(t, x, \tilde{z}) & =\boldsymbol{f}(\tilde{\boldsymbol{z}} \sigma(t, x)) \quad \text { and } \\
\boldsymbol{f}(\boldsymbol{z}) & =-\frac{1}{2} \boldsymbol{v}^{2}+\frac{1}{2} \boldsymbol{A}[\boldsymbol{\mu}]^{2}-\boldsymbol{A}[\boldsymbol{\mu}] \boldsymbol{\mu} \quad \text { with } \boldsymbol{z}=(\boldsymbol{\mu}, \boldsymbol{v}) .
\end{aligned}
$$

We will use Theorem 2.14 to establish the existence of a Höldearian solution $(\tilde{\boldsymbol{v}}, \tilde{\boldsymbol{w}})$. Then $(\tilde{\boldsymbol{v}}, \tilde{\boldsymbol{w}} \sigma)$ is a Höldearian solution of (3.1). 
Let us first verify the condition (AB). Let $\sigma_{i}, i=1,2$, be the $i$ th column vector of $\sigma$. Denote $\tilde{\mu}=\tilde{z} \sigma_{1}$ and $\tilde{v}=\tilde{z} \sigma_{2}$. Then

$$
\tilde{f}=-\frac{1}{2} \tilde{v}^{2}+\frac{1}{2} A[\tilde{\mu}]^{2}-A[\tilde{\mu}] \tilde{\mu} .
$$

Let $\left(\mathfrak{e}_{1}, \ldots, \mathfrak{e}_{N}\right)$ be the standard Euclidean basis of $\mathbb{R}^{N}$, and $\boldsymbol{a}_{N+1}=\left(\alpha^{1}, \ldots, \alpha^{N}\right)$ where the sequence of constants $\left\{\alpha^{i}\right\}$ appears after (3.1). Then the set $\left(-\mathfrak{e}_{1}, \ldots\right.$, $\left.-\mathfrak{e}_{N}, \boldsymbol{a}_{N+1}\right)$ positively span $\mathbb{R}^{N}$. Moreover,

$$
\begin{aligned}
-\mathfrak{e}_{i}^{\top} \tilde{\boldsymbol{f}} & \leq \frac{1}{2}\left(\tilde{\mu}^{i}\right)^{2}+\frac{1}{2}\left(\tilde{v}^{i}\right)^{2} \quad \text { for } 1 \leq i \leq N \quad \text { and } \\
\boldsymbol{a}_{N+1}^{\top} \tilde{\boldsymbol{f}} & =-\frac{1}{2} \boldsymbol{A}\left[\tilde{\boldsymbol{v}}^{2}\right]-\frac{1}{2} \boldsymbol{A}[\tilde{\boldsymbol{\mu}}]^{2} \leq 0 .
\end{aligned}
$$

In order to verify the condition (BF), let us introduce an invertible linear transformation on $\mathbb{R}^{N}$ via

$$
\bar{Y}^{i}=\tilde{Y}^{i}-\tilde{Y}^{N}, \quad i=1, \ldots, N-1, \quad \bar{Y}^{N}=\tilde{Y}^{N},
$$

and $\overline{\boldsymbol{\mu}}, \overline{\boldsymbol{v}}$ and $\overline{\boldsymbol{g}}$ in a similar manner. A simple calculation reveals the dynamics of $\overline{\boldsymbol{Y}}$ as

$$
d \overline{\boldsymbol{Y}}_{t}=\overline{\boldsymbol{\mu}}_{t} d B_{t}+\overline{\boldsymbol{v}}_{t} d B_{t}^{\perp}-\overline{\boldsymbol{f}}\left(\overline{\boldsymbol{\mu}}_{t}, \overline{\boldsymbol{v}}_{t}\right) d t, \quad \overline{\boldsymbol{Y}}_{T}=\overline{\boldsymbol{g}}\left(B_{T}, W_{T}\right),
$$

where $\bar{f}$ is given by

$$
\begin{aligned}
& \bar{f}^{i}=-\frac{1}{2} \bar{v}^{i}\left(\bar{v}^{i}+2 \bar{v}^{N}\right)-\bar{\mu}^{i}\left(\sum_{j=1}^{N-1} \alpha^{j} \bar{\mu}^{j}+\bar{\mu}^{N}\right), \quad i=1, \ldots, N-1, \quad \text { and } \\
& \bar{f}^{N}=-\frac{1}{2}\left(\bar{v}^{N}\right)^{2}+\frac{1}{2}\left(\sum_{j=1}^{N-1} \alpha^{j} \bar{\mu}^{j}+\bar{\mu}^{N}\right)^{2}-\bar{\mu}^{N}\left(\sum_{j=1}^{N-1} \alpha^{j} \bar{\mu}^{j}+\bar{\mu}^{N}\right) .
\end{aligned}
$$

Using this explicit expression, one easily checks that $\bar{f}$ satisfies the condition (BF) of Definition 2.10. On the other hand, since $\tilde{f}$ already satisfies the condition (AB), after the linear transformation of $\mathbb{R}^{N}, \bar{f}$ satisfies (AB) as well (cf. Remark 2.13). Therefore, the existence and uniqueness of a bounded continuous solution to (5.12) [hence (5.11) and (3.1)] follows from Theorem 2.14. Finally, when the terminal condition is bounded, combining Theorem 2.9 part (1) and [40], Theorem 1.6(2) $\rightarrow$ (1), we confirm the existence of an equilibrium. Conversely, any equilibrium with continuous certainty equivalence functions corresponds to a continuous Markovian solution of (3.1), which is already proven to be unique. 
5.4.2. Proof of Proposition 3.4. We define the approximated driver $\boldsymbol{f}^{m}$ by

$$
\left(\boldsymbol{f}^{m}(\boldsymbol{y}, \boldsymbol{z})\right)^{k}=\frac{1}{2} \sum_{i, j} \Gamma_{i j}^{k}(\boldsymbol{y})\left(\Pi^{m}(\boldsymbol{z})^{\top} \Pi^{m}(\boldsymbol{z})\right)_{i j} \quad \text { for } \boldsymbol{y} \in \mathbb{R}^{N}, \boldsymbol{z} \in \mathbb{R}^{N \times d}
$$

where $\Pi^{m}(\boldsymbol{z})=\frac{|z| \wedge m}{|z|} \boldsymbol{z}$ for $m \in \mathbb{N}$. Also, we construct a sequence $\left(\boldsymbol{g}^{m}\right)$ of Lipschitz approximation of $\boldsymbol{g}$ as in Proposition 5.4. Mollification does not increase the $\mathbb{L}^{\infty}$-norm and the set sub-level set $M_{0}=\phi^{-1}((-\infty, 0])$ is convex; therefore, the images of all $\boldsymbol{g}^{m}$ remain inside $M_{0}$, that is, $\phi^{-1}\left(\boldsymbol{g}^{m}(x)\right) \leq 0$, for all $x$ and $m$.

The globally-Lipschitz structure of its ingredients implies that the approximated system

$$
d \boldsymbol{Y}_{t}^{m}=-\boldsymbol{f}^{m}\left(\boldsymbol{Y}_{t}^{m}, \boldsymbol{Z}_{t}^{m}\right) d t+\boldsymbol{Z}_{t}^{m} d W_{t}, \quad \boldsymbol{Y}_{T}^{m}=\boldsymbol{g}^{m}\left(W_{T}\right),
$$

admits a unique Hölderian solution $\left(\boldsymbol{v}^{m}, \boldsymbol{w}^{m}\right)$. To show that the sequence $\left\{\boldsymbol{v}^{m}\right\}$ is uniformly bounded, more precisely, that $\boldsymbol{v}^{m} \in M_{0}$ for all $m$, we define the stopping time $\tau=\inf \left\{s \geq t: \phi\left(\boldsymbol{Y}_{s}^{m}\right) \leq \varepsilon\right\} \wedge T$, for given $t \in[0, T]$ and $\varepsilon>0$. By Itô's formula, we obtain

$$
\begin{aligned}
& \mathbb{E}_{t}\left[\phi\left(\boldsymbol{Y}^{m}(\tau)\right)\right]-\phi\left(\boldsymbol{Y}_{t}^{m}\right) \\
&=\mathbb{E}_{t}\left[\int_{t}^{\tau} \frac{1}{2} D^{2} \phi\left(\boldsymbol{Y}_{s}^{m}\right):\left(\boldsymbol{Z}_{s}^{m}\right)^{\top} \boldsymbol{Z}_{s}^{m}\right. \\
&\left.\quad-\frac{1}{2} D \phi\left(\boldsymbol{Y}_{s}^{m}\right) \Gamma\left(\boldsymbol{Y}_{s}^{m}\right) \Pi^{m}\left(\boldsymbol{Z}_{s}^{m}\right)^{\top} \Pi^{m}\left(\boldsymbol{Z}_{s}^{m}\right) d s\right] \\
&=\frac{1}{2} \mathbb{E}_{t}\left[\int _ { t } ^ { \tau } \left(\operatorname{Hess} \phi\left(\Pi^{m}\left(\boldsymbol{Z}_{s}^{m}\right), \Pi^{m}\left(\boldsymbol{Z}_{s}^{m}\right)\right)\right.\right. \\
&\left.\left.+\frac{1}{2} \frac{\left|\boldsymbol{Z}_{s}\right|-\left|\boldsymbol{Z}_{s}\right| \wedge m}{\left|\boldsymbol{Z}_{s}\right|} D^{2} \phi\left(\boldsymbol{Y}_{s}^{m}\right)\left(\boldsymbol{Z}_{s}^{m}\right)^{\top} \boldsymbol{Z}_{s}^{m}\right) d s\right],
\end{aligned}
$$

where the local martingale term can be dealt with by stopping, using the fact that $D \phi$ is bounded on compacts and $Z^{m} \in H^{2}$. Double convexity of $\phi$ implies that both terms inside the expectation above are nonnegative, so that

$$
\phi\left(\boldsymbol{Y}_{t}^{m}\right) \leq \mathbb{E}_{t}\left[\phi\left(\boldsymbol{Y}_{\tau}^{m}\right)\right], \quad \text { a.s. }
$$

Since $\phi\left(\boldsymbol{Y}_{T}^{m}\right) \leq 0<\varepsilon$ the stopping time $\tau$ gets realized strictly before $T$. Therefore, the right-hand side above is bounded from above by $\varepsilon$, immediately implying that $\phi\left(\boldsymbol{Y}_{t}^{m}\right) \leq \varepsilon$, a.s., and establishing the claim about boundedness of $\boldsymbol{v}^{m}$.

Once the a priori boundedness of the approximating sequence $\left\{\boldsymbol{v}^{m}\right\}$ is established, we can use the strict geodesic convexity of $\phi$ on some neighborhood of $M_{0}$ to conclude that $\phi$ can be suitably redefined on a complement of a neighborhood of $M_{0}$ to serve, together with $k=0$, as a $c$-Lyapunov pair, for large enough $c$. Then the existence of a locally Hölderian solution to (3.2), with $\boldsymbol{Y}_{T}=\boldsymbol{g}\left(W_{T}\right)$, readily follows from Theorem 2.8. 
5.4.3. Proof of Proposition 3.6. Let us first $\operatorname{argue}$ that a solution $(\boldsymbol{Y}, \boldsymbol{Z})$ with $(\hat{\boldsymbol{\mu}}(\boldsymbol{Z}), \hat{\boldsymbol{v}}(\boldsymbol{Z})) \in$ bmo $^{2}$ to (3.5) corresponds to a Nash equilibrium. For a given $\boldsymbol{\mu} \in$ bmo, we consider the process

$$
\tilde{Y}_{t}^{1}=\mathbb{E}_{t}^{\mu, \hat{v}}\left[\int_{t}^{T}\left(h^{1}\left(X_{u}\right)+\frac{1}{2}\left|\boldsymbol{\mu}_{u}\right|^{2}+\theta \boldsymbol{\mu}_{u}^{\top} \hat{\boldsymbol{v}}_{u}\right) d u+g^{1}\left(X_{T}\right)\right] .
$$

Thanks to the fact that $h^{1}$ and $g^{1}$ are both bounded and $\boldsymbol{\mu}, \hat{\boldsymbol{v}} \in$ bmo, $\tilde{Y}^{1}$ is bounded. Since $X$ generates the same filtration as $W$, the martingale representation implies the existence of a process $\tilde{\boldsymbol{Z}}^{1} \in \mathcal{P}^{2}$ such that

$$
\tilde{Y}_{t}^{1}=g^{1}\left(X_{T}\right)+\int_{t}^{T}\left(h^{1}\left(X_{u}\right)+\frac{1}{2}\left|\boldsymbol{\mu}_{u}\right|^{2}+\theta \boldsymbol{\mu}_{u}^{\top} \hat{\boldsymbol{v}}_{u}\right) d u-\int_{t}^{T} \tilde{\boldsymbol{Z}}_{u}^{1} d W_{u}^{\mu, \hat{v}} .
$$

If we subtract the corresponding component $Y^{1}$ of the solution $\boldsymbol{Y}$ of (3.5) from it, we obtain

$$
\tilde{Y}_{0}^{1}-Y_{0}^{1}=\int_{0}^{T}\left(L^{1}\left(\boldsymbol{\mu}_{u}, \hat{\boldsymbol{v}}_{u}, \boldsymbol{Z}_{u}\right)-L^{1}\left(\hat{\boldsymbol{\mu}}_{u}, \hat{\boldsymbol{v}}_{u}, \boldsymbol{Z}_{u}\right)\right) d u-\int_{0}^{T}\left(\tilde{\boldsymbol{Z}}_{u}^{1}-\boldsymbol{Z}_{u}^{1}\right) d W_{u}^{\mu, \hat{v}} .
$$

Since $\hat{\boldsymbol{\mu}}$ is the minimizer of $L^{1}(\cdot, \hat{\boldsymbol{v}}, \boldsymbol{Z})$, and both $Y^{1}$ and $\tilde{Y}^{1}$ are bounded, a localization argument yields $\tilde{Y}_{0}^{1} \geq \hat{Y}_{0}^{1}$, confirming the first inequality in (3.3). A similar argument applies to the cost of the second player as well.

For the existence and uniqueness of $(\boldsymbol{Y}, \boldsymbol{Z})$, we verify all conditions in Theorem 2.14 and introduce an invertible linear transformation on $\mathbb{R}^{2}$ via $\tilde{y}^{1}=y^{1}-y^{2}$ and $\tilde{y}^{2}=y^{2}$. Define $\tilde{\boldsymbol{z}}, \tilde{\boldsymbol{g}}$ similarly, and consider the BSDE

$$
d \tilde{\boldsymbol{Y}}_{t}=-\tilde{\boldsymbol{f}}\left(X_{t}, \tilde{\boldsymbol{Z}}_{t}\right) d t+\tilde{\boldsymbol{Z}}_{t} d W_{t}, \quad \tilde{\boldsymbol{Y}}_{T}=\tilde{\boldsymbol{g}}\left(X_{T}\right),
$$

where

$$
\tilde{f}^{1}(x, \tilde{z})=\frac{2 \theta-1}{2(1+\theta)(1-\theta)} \tilde{\boldsymbol{z}}^{1} \cdot\left(\tilde{z}^{1}+2 \tilde{z}^{2}\right)+h^{1}(x)-h^{2}(x) \quad \text { and } \quad \tilde{f}^{2}=f^{2} .
$$

Using this explicit expression, one easily checks that $\tilde{f}$ satisfies the condition (BF).

Next, we show that $\boldsymbol{f}$ satisfies the condition (wAB), hence $\tilde{f}$ satisfies the same condition as well. To this end, calculation shows that

$$
\begin{aligned}
L^{1}(z)= & -\frac{\theta^{2}}{2(1+\theta)^{2}(1-\theta)^{2}}\left|z^{1}+z^{2}\right|^{2} \\
& -\frac{1-2 \theta}{2(1-\theta)^{2}(1+\theta)} z^{1} \cdot\left((1-\theta) z^{1}+2 z^{2}\right) \\
\leq & -\frac{1-2 \theta}{2(1-\theta)^{2}(1+\theta)} z^{1} \cdot\left((1-\theta) z^{1}+2 z^{2}\right) .
\end{aligned}
$$

A similar inequality holds for $L^{2}(\boldsymbol{z})$. Therefore, using the fact that $\boldsymbol{h}$ is bounded, we obtain functions $\boldsymbol{L}_{i}$ such that $\mathfrak{e}_{i}^{\top} \boldsymbol{f} \leq h^{i}+\mathfrak{e}_{i}^{\top} \boldsymbol{z} \boldsymbol{L}_{i}$ for $i=1,2$. 
When $\theta \leq 1 / 2$, using the first identity above, we obtain

$$
\begin{aligned}
L^{1}(z)+L^{2}(z) & =\frac{\theta^{2}+\theta-1}{(1+\theta)^{2}(1-\theta)^{2}}\left|z^{1}+z^{2}\right|^{2}+\frac{1-2 \theta}{2(1-\theta)^{2}}\left(\left|z^{1}\right|^{2}+\left|z^{2}\right|^{2}\right) \\
& \geq \frac{\theta^{2}+\theta-1}{(1+\theta)^{2}(1-\theta)^{2}}\left|z^{1}+z^{2}\right|^{2} .
\end{aligned}
$$

Hence, for $\boldsymbol{a}_{3}=(-1,-1)$, we have

$$
\boldsymbol{a}_{3}^{\top} \boldsymbol{f} \leq 2\left\|h^{1}+h^{2}\right\|_{\mathbb{L}^{\infty}}+\frac{\left|\theta^{2}+\theta-1\right|}{(1+\theta)^{2}(1-\theta)^{2}}\left|z^{1}+z^{2}\right|^{2} .
$$

As a result, $f$ satisfies the condition (wAB) with the positively spanning set $\left(\mathfrak{e}_{1}, \mathfrak{e}_{2}, \boldsymbol{a}_{3}\right)$.

When $\theta>1$, consider $\boldsymbol{a}_{3}=(-\theta, 1)$ and $\boldsymbol{a}_{4}=(1,-\theta)$. We have from (3.4) that

$$
\begin{aligned}
& \hat{\boldsymbol{\mu}}=\frac{1}{(1-\theta)(1+\theta)}\left(\theta z^{2}-z^{1}\right)=\frac{1}{(\theta-1)(1+\theta)} \boldsymbol{a}_{4}^{\top} \boldsymbol{z} \quad \text { and } \\
& \hat{\boldsymbol{v}}=\frac{1}{(1-\theta)(1+\theta)}\left(\theta z^{1}-z^{2}\right)=\frac{1}{(\theta-1)(1+\theta)} \boldsymbol{a}_{3}^{\top} z .
\end{aligned}
$$

On the other hand, for $\theta>1$, we have

$$
\begin{aligned}
\boldsymbol{a}_{3}^{\top} \boldsymbol{L} & =-\theta L^{1}+L^{2}=-\frac{\theta}{2}|\hat{\boldsymbol{\mu}}+\hat{\boldsymbol{v}}|^{2}+\left(\frac{1}{2}-\theta\right)|\hat{\boldsymbol{v}}|^{2}+(2 \theta-1) \hat{\boldsymbol{v}}^{\top}\left(\hat{\boldsymbol{\mu}}+\frac{\theta+2}{2} \hat{\boldsymbol{v}}\right) \\
& \leq \frac{2 \theta-1}{(\theta-1)^{2}(1+\theta)^{2}}\left(\boldsymbol{a}_{3}^{\top} \tilde{\boldsymbol{z}}\right)^{\top}\left(\frac{\theta+2}{2} \boldsymbol{a}_{3}+\boldsymbol{a}_{4}\right)^{\top} \boldsymbol{z} .
\end{aligned}
$$

A similar inequality holds for $\boldsymbol{a}_{4}^{\top} \boldsymbol{L}$. Combining the previous two estimates together with boundedness of $\boldsymbol{h}$, we confirm that $\boldsymbol{f}$ satisfies the condition (wAB) with the set of vectors $\left(\mathfrak{e}_{1}, \mathfrak{e}_{2}, \boldsymbol{a}_{3}, \boldsymbol{a}_{4}\right)$, which positively span $\mathbb{R}^{2}$ when $\theta>1$.

Finally, we conclude from Theorem 2.14 that the system (5.13) [hence (3.5)] admits a unique bounded continuous solution.

5.4.4. Proof of Proposition 3.7. It is clear that the generator of system (3.6) satisfies the condition (BF) and (wAB) when $\boldsymbol{g}$ is bounded [(AB) when $\boldsymbol{g}$ is unbounded]. Then the existence (and uniqueness for bounded $\boldsymbol{g}$ ) readily follows from Theorem 2.14. Given the bounded continuous solution, $Z=\boldsymbol{w}(\cdot, X) \in$ bmo, hence [26], Proposition 5.1, concludes that $(\hat{\boldsymbol{\mu}}, \hat{\boldsymbol{v}})$ is a Nash equilibrium with value $\left[\exp \left(v^{1}\right), \exp \left(v^{2}\right)\right]$.

Acknowledgment. The first author is grateful to Ying Hu for inspiring discussions. 


\section{REFERENCES}

[1] Aronson, D. G. (1967). Bounds for the fundamental solution of a parabolic equation. Bull. Amer. Math. Soc. 73 890-896. MR0217444

[2] Aubin, T. (1998). Some Nonlinear Problems in Riemannian Geometry. Springer Monographs in Mathematics. Springer, Berlin. MR1636569

[3] Bally, V. and Matoussi, A. (2001). Weak solutions for SPDEs and backward doubly stochastic differential equations. J. Theoret. Probab. 14 125-164. MR1822898

[4] Barles, G. and Lesigne, E. (1997). SDE, BSDE and PDE. In Backward Stochastic Differential Equations (Paris, 1995-1996). Pitman Res. Notes Math. Ser. 364 47-80. Longman, Harlow. MR1752675

[5] Barrieu, P. and El Karoui, N. (2013). Monotone stability of quadratic semimartingales with applications to unbounded general quadratic BSDEs. Ann. Probab. 41 1831-1863. MR3098060

[6] Bensoussan, A. and Frehse, J. (2000). Stochastic games for $N$ players. J. Optim. Theory Appl. 105 543-565. Special Issue in honor of Professor David G. Luenberger. MR1783877

[7] Bensoussan, A. and Frehse, J. (2002). Smooth solutions of systems of quasilinear parabolic equations. ESAIM Control Optim. Calc. Var. 8 169-193. MR1932949

[8] Bismut, J.-M. (1973). Conjugate convex functions in optimal stochastic control. J. Math. Anal. Appl. 44 384-404. MR0329726

[9] BLACHE, F. (2005). Backward stochastic differential equations on manifolds. Probab. Theory Related Fields 132 391-437. MR2197107

[10] Blache, F. (2006). Backward stochastic differential equations on manifolds. II. Probab. Theory Related Fields 136 234-262. MR2240788

[11] BRIAND, P. and EliE, R. (2013). A simple constructive approach to quadratic BSDEs with or without delay. Stochastic Process. Appl. 123 2921-2939. MR3062430

[12] BRIAnd, P. and HU, Y. (2006). BSDE with quadratic growth and unbounded terminal value. Probab. Theory Related Fields 136 604-618. MR2257138

[13] BRIAND, P. and Hu, Y. (2008). Quadratic BSDEs with convex generators and unbounded terminal conditions. Probab. Theory Related Fields 141 543-567. MR2391164

[14] Çetin, U. and Danilova, A. (2016). Markovian Nash equilibrium in financial markets with asymmetric information and related forward-backward systems. Ann. Appl. Probab. 26 1996-2029. MR3543888

[15] Chang, K.-C., Ding, W. Y. and Ye, R. (1992). Finite-time blow-up of the heat flow of harmonic maps from surfaces. J. Differential Geom. 36 507-515. MR1180392

[16] Cheridito, P., Horst, U., Kupper, M. and Pirvu, T. A. (2016). Equilibrium pricing in incomplete markets under translation invariant preferences. Math. Oper. Res. 41 174-195. MR3465747

[17] Cheridito, P. and NAM, K. (2014). BSDEs with terminal conditions that have bounded Malliavin derivative. J. Funct. Anal. 266 1257-1285. MR3146818

[18] Cheridito, P. and NAM, K. (2015). Multidimensional quadratic and subquadratic BSDEs with special structure. Stochastics 87 871-884. MR3390237

[19] Chitashvili, R. and Mania, M. (1997). On functions transforming a Wiener process into a semimartingale. Probab. Theory Related Fields 109 57-76. MR1469920

[20] ChOI, J. H. and LARSEN, K. (2015). Taylor approximation of incomplete Radner equilibrium models. Finance Stoch. 19 653-679. MR3369442

[21] DARLing, R. W. R. (1995). Constructing gamma-martingales with prescribed limit, using backwards SDE. Ann. Probab. 23 1234-1261. MR1349170

[22] Davis, C. (1954). Theory of positive linear dependence. Amer. J. Math. 76 733-746. MR0064011 
[23] Delarue, F. (2003). Estimates of the solutions of a system of quasi-linear PDEs. A probabilistic scheme. In Séminaire de Probabilités XXXVII. Lecture Notes in Math. 1832 290-332. Springer, Berlin. MR2053051

[24] Delbaen, F., Hu, Y. and BAO, X. (2011). Backward SDEs with superquadratic growth. Probab. Theory Related Fields 150 145-192. MR2800907

[25] Eells, J. JR. and SAMPSON, J. H. (1964). Harmonic mappings of Riemannian manifolds. Amer. J. Math. 86 109-160. MR0164306

[26] El-KARoui, N. and HAMADÈnE, S. (2003). BSDEs and risk-sensitive control, zero-sum and nonzero-sum game problems of stochastic functional differential equations. Stochastic Process. Appl. 107 145-169. MR1995925

[27] El Karoui, N., Peng, S. and QueneZ, M. C. (1997). Backward stochastic differential equations in finance. Math. Finance 7 1-71. MR1434407

[28] ÉMERY, M. (1989). Stochastic Calculus in Manifolds. Springer, Berlin. MR1030543

[29] EspinosA, G.-E. and TouZI, N. (2015). Optimal investment under relative performance concerns. Math. Finance 25 221-257. MR3321249

[30] Evans, L. C. (1998). Partial Differential Equations. Graduate Studies in Mathematics 19. Amer. Math. Soc., Providence, RI. MR1625845

[31] Frehse, J. (1988). Remarks on diagonal elliptic systems. In Partial Differential Equations and Calculus of Variations. Lecture Notes in Math. 1357 198-210. Springer, Berlin. MR0976236

[32] FREI, C. (2014). Splitting multidimensional BSDEs and finding local equilibria. Stochastic Process. Appl. 124 2654-2671. MR3200729

[33] FreI, C. and Dos ReIS, G. (2011). A financial market with interacting investors: Does an equilibrium exist? Math. Financ. Econ. 4 161-182. MR2796281

[34] Friedman, A. (1964). Partial Differential Equations of Parabolic Type. Prentice-Hall, Englewood Cliffs, NJ. MR0181836

[35] Hsu, E. P. (2002). Stochastic Analysis on Manifolds. Graduate Studies in Mathematics 38. Amer. Math. Soc., Providence, RI. MR1882015

[36] HU, Y. and PENG, S. (2006). On the comparison theorem for multidimensional BSDEs. $C$. $R$. Math. Acad. Sci. Paris 343 135-140. MR2243308

[37] HU, Y. and TANG, S. (2016). Multi-dimensional backward stochastic differential equations of diagonally quadratic generators. Stochastic Process. Appl. 126 1066-1086. MR3461191

[38] IL'In, A. M., KALAŠNiKOV, A. S. and OLEĬNIK, O. A. (1962). Second-order linear equations of parabolic type. Uspekhi Mat. Nauk 17 3-146. MR0138888

[39] John, F. (1978). Partial Differential Equations, 3rd ed. Applied Mathematical Sciences 1. Springer, New York. MR0514404

[40] KardaraS, C., XING, H. and ŽITKOvić (2015). Incomplete stochastic equilibria with exponential utilities: Close to Pareto optimality. Working paper.

[41] Kendall, W. S. (1990). Probability, convexity, and harmonic maps with small image. I. Uniqueness and fine existence. Proc. Lond. Math. Soc. (3) 61 371-406. MR1063050

[42] KobYlanski, M. (2000). Backward stochastic differential equations and partial differential equations with quadratic growth. Ann. Probab. 28 558-602. MR1782267

[43] Kramkov, D. and Pulido, S. (2016). A system of quadratic BSDEs arising in a price impact model. Ann. Appl. Probab. 26 794-817. MR3476625

[44] Ladyženskaja, O. A., Solonnikov, V. A. and Ural'ceva, N. N. (1968). Linear and Quasilinear Equations of Parabolic Type. Translations of Mathematical Monographs 23. Amer. Math. Soc., Providence, RI. Translated from the Russian by S. Smith. MR0241822

[45] LEJAY, A. (2002). BSDE driven by Dirichlet process and semi-linear parabolic PDE. Application to homogenization. Stochastic Process. Appl. 97 1-39. MR1870718

[46] Lepeltier, J. P. and SAN MARTin, J. (1997). Backward stochastic differential equations with continuous coefficient. Statist. Probab. Lett. 32 425-430. MR1602231 
[47] Lieberman, G. M. (1996). Second Order Parabolic Differential Equations. World Scientific, River Edge, NJ. MR1465184

[48] Matoussi, A. and XU, M. (2008). Sobolev solution for semilinear PDE with obstacle under monotonicity condition. Electron. J. Probab. 13 1035-1067. MR2424986

[49] PARdouX, É. and Peng, S. (1992). Backward stochastic differential equations and quasilinear parabolic partial differential equations. In Stochastic Partial Differential Equations and Their Applications (Charlotte, NC, 1991). Lect. Notes Control Inf. Sci. 176 200-217. Springer, Berlin. MR1176785

[50] PARdouX, É. and PENG, S. G. (1990). Adapted solution of a backward stochastic differential equation. Systems Control Lett. 14 55-61. MR1037747

[51] Peng, S. (1999). Open problems on backward stochastic differential equations. In Control of Distributed Parameter and Stochastic Systems (Hangzhou, 1998) 265-273. Kluwer Academic, Boston, MA. MR1777419

[52] Stroock, D. W. and Varadhan, S. R. S. (2006). Multidimensional Diffusion Processes. Springer, Berlin. Reprint of the 1997 edition. MR2190038

[53] Struwe, M. (1981). On the Hölder continuity of bounded weak solutions of quasilinear parabolic systems. Manuscripta Math. 35 125-145. MR0627929

[54] Subrahmanyam, A. (1991). Risk aversion, market liquidity, and price efficiency. Rev. Financ. Stud. 4 417-441.

[55] TANG, S. (2003). General linear quadratic optimal stochastic control problems with random coefficients: Linear stochastic Hamilton systems and backward stochastic Riccati equations. SIAM J. Control Optim. 42 53-75. MR1982735

[56] Tevzadze, R. (2008). Solvability of backward stochastic differential equations with quadratic growth. Stochastic Process. Appl. 118 503-515. MR2389055

[57] UdRIŞTE, C. (1994). Convex Functions and Optimization Methods on Riemannian Manifolds. Mathematics and Its Applications 297. Kluwer Academic, Dordrecht. MR1326607

[58] WidmAn, K.-O. (1971). Hölder continuity of solutions of elliptic systems. Manuscripta Math. 5 299-308. MR0296484

[59] ZhaO, Y. (2012). Stochastic equilibria in a general class of incomplete Brownian market environments. Ph.D. thesis, Univ. Texas at Austin.

[60] Žıtrković, G. (2012). An example of a stochastic equilibrium with incomplete markets. Finance Stoch. 16 177-206. MR2903621

DEPARTMENT OF STATISTICS

LONDON SCHOOL OF ECONOMICS

LONDON

UNITED KINGDOM

E-MAIL: h.xing@1se.ac.uk
DEPARTMENT OF MATHEMATICS

UNIVERSITY OF TEXAS AT AUSTIN AUSTIN, TEXAs 78712

USA

E-MAIL: gordanz@math.utexas.edu 